\title{
Results of the BETS Survey of the CC Cryostat
}

Engineering Note \#3740.214-EN-127

This Engineering Note presents results of dimensional surveys of the CC Cryostat. The surveys were performed by members of the Fermilab Alignment Group using a computerized optical system known as BETS. The coordinate system used is described on page 1 of the note. Locations of the support bosses in the inner vessel are given on pages 2 and 3 . The bosses control the position of the module array within the cryostat. Locations of the center cylinders (bores) and bypass tubes in both vessels are given on pages 2 and 4 through 6. Elevations and locations of the nozzles on top of the cryostat are given on page 9 . Measurements of the stack-up heights of the support stanchions are given on page 13. Raw BETS data are included in Appendix A of the Note.

This engineering note contains 18 pages and Appendix A contains 39 pages.

Issued: January 7, 1988

Originator: Richard D. Luther $Q D D$. 
ex $11 / 2 / 87$

2

Server Dropt

(coumenno ro Cloces. Srsigm

show ow Alan. Ame-)

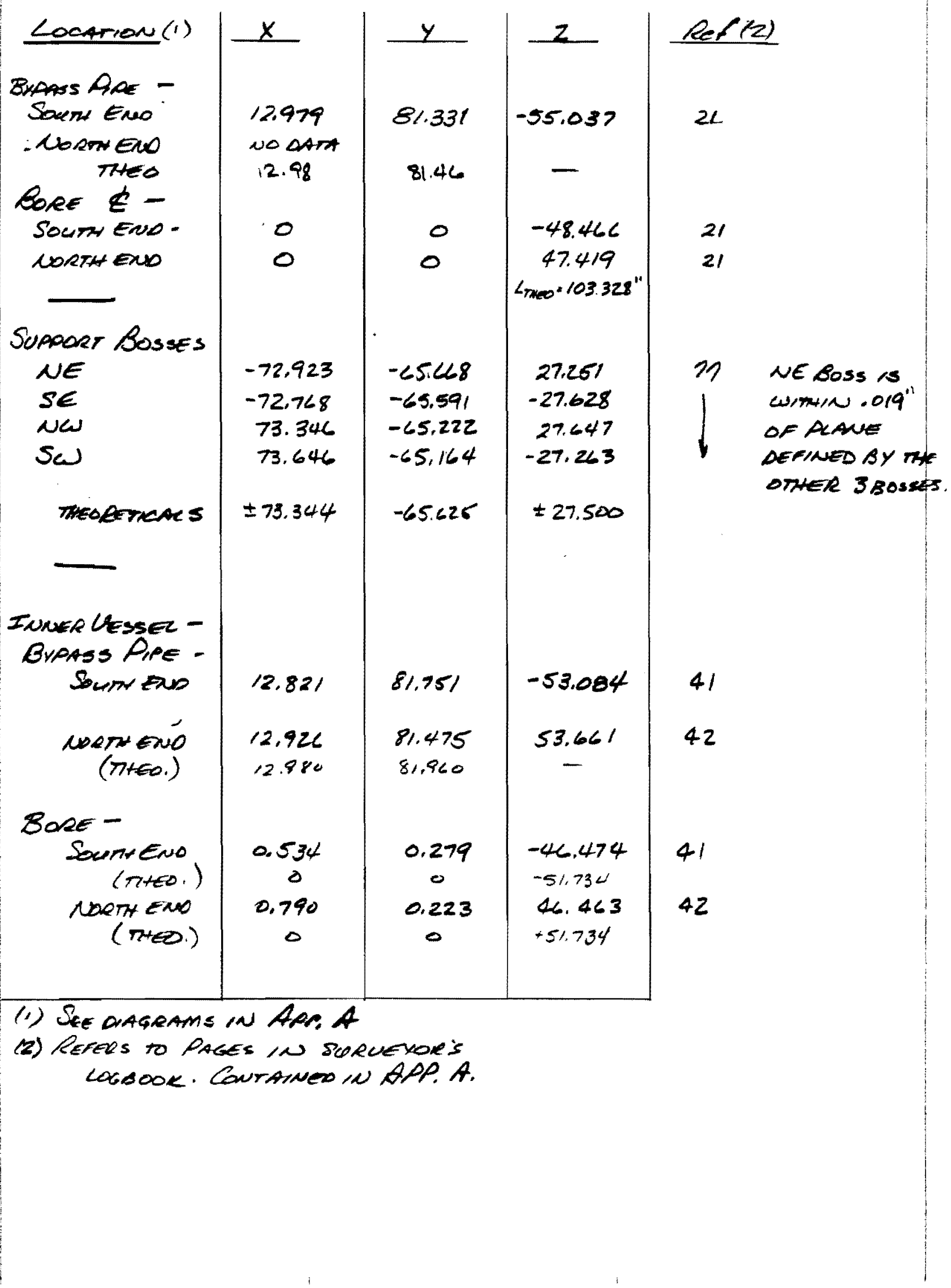




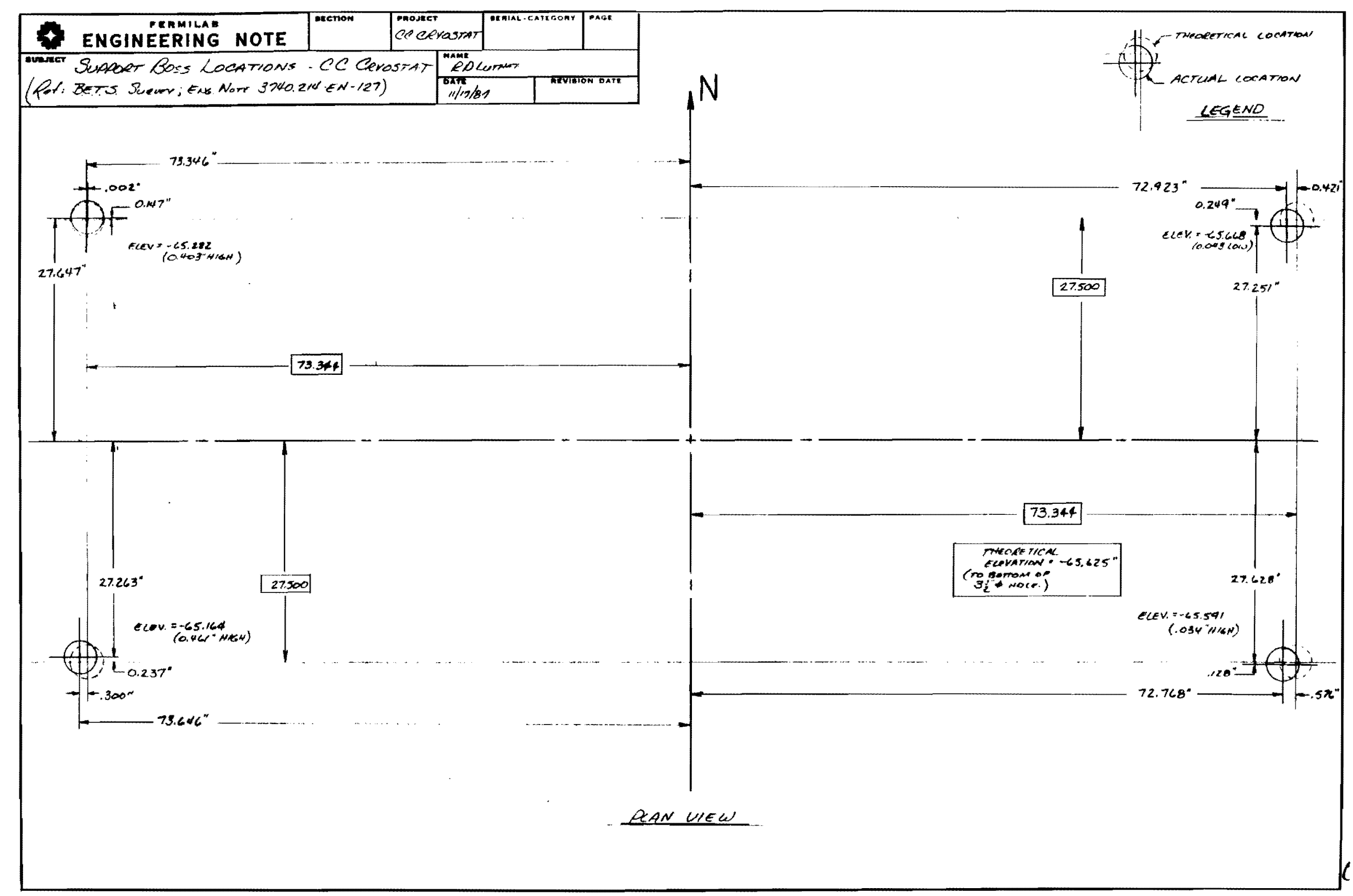




\section{Suruey Resuers}

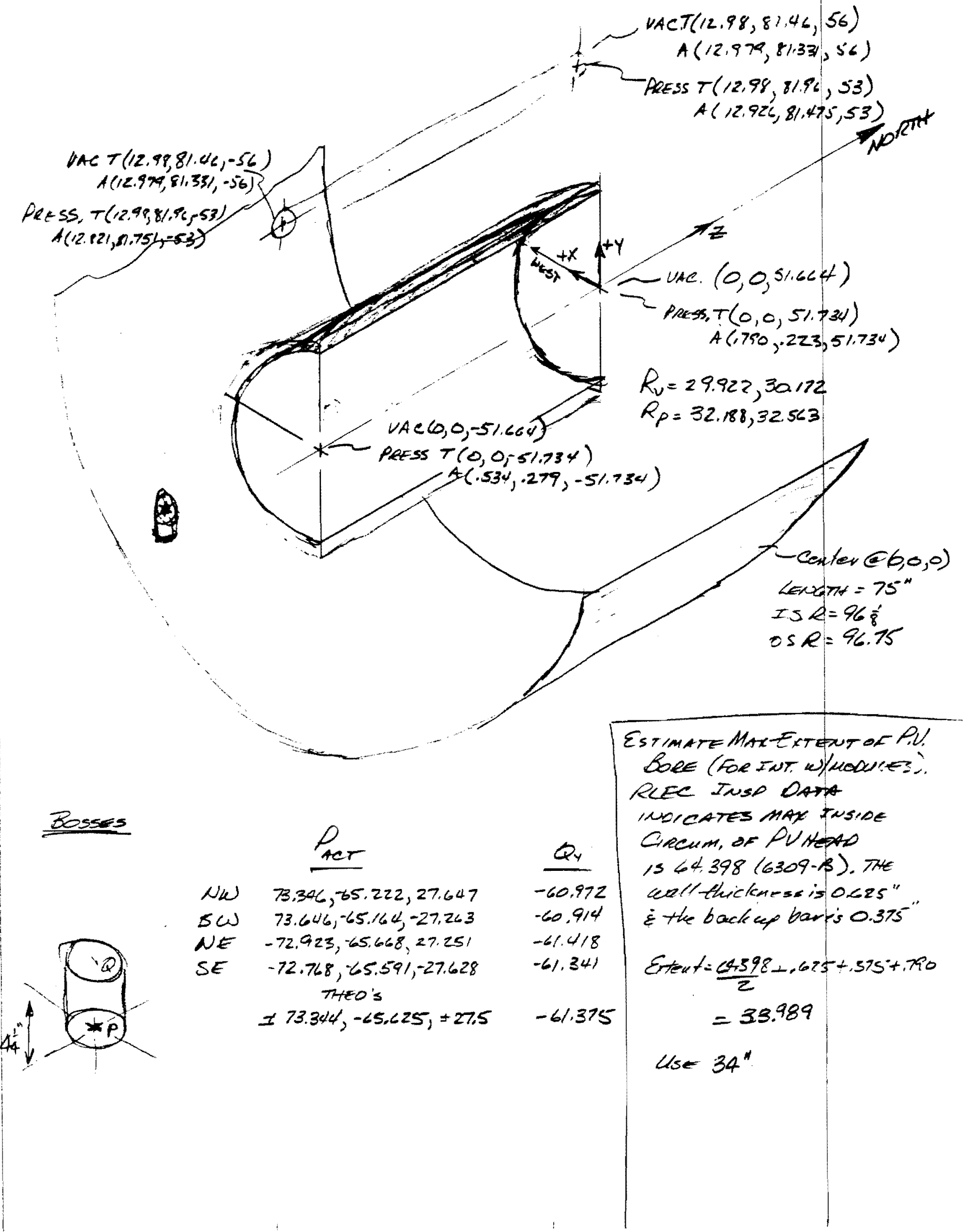




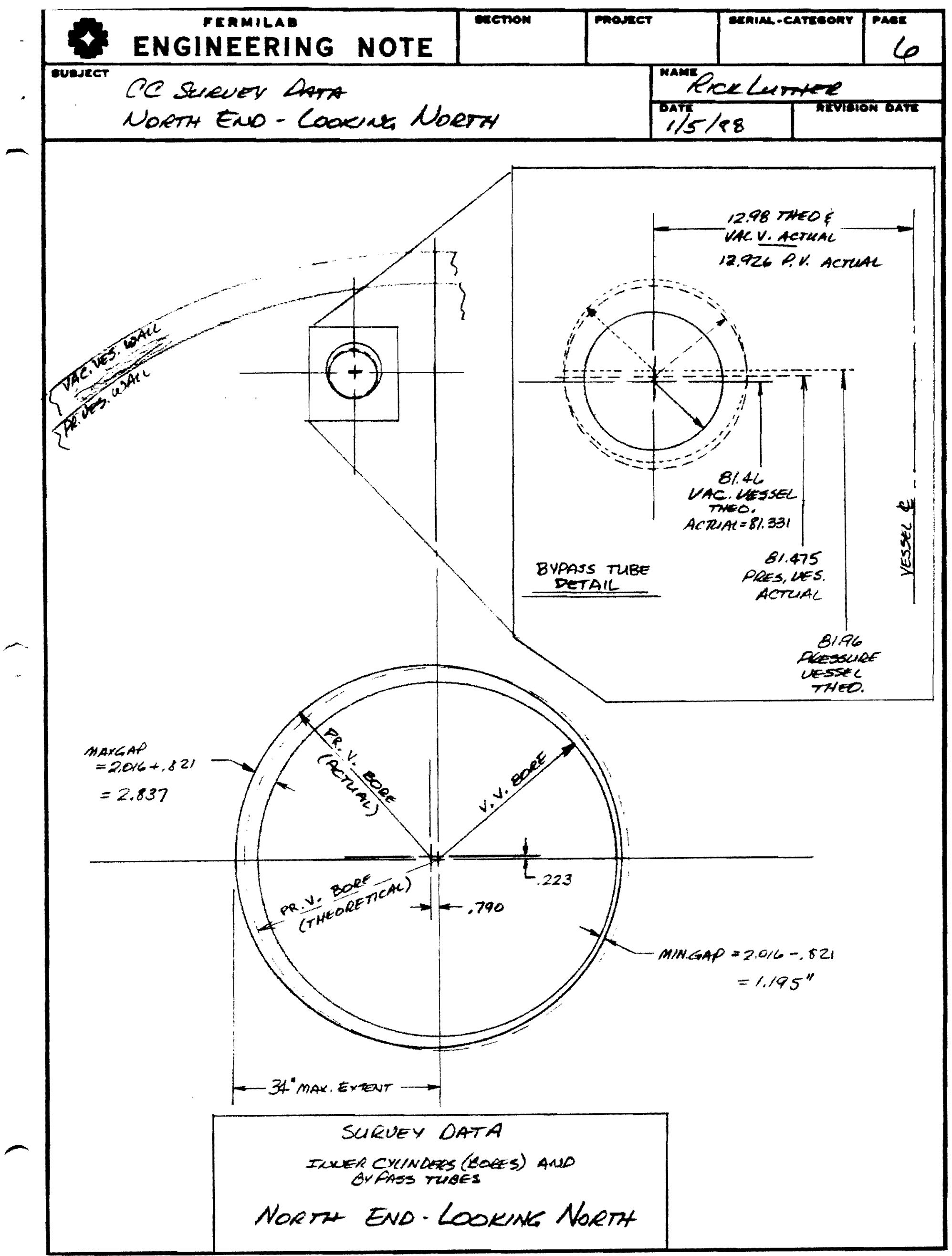




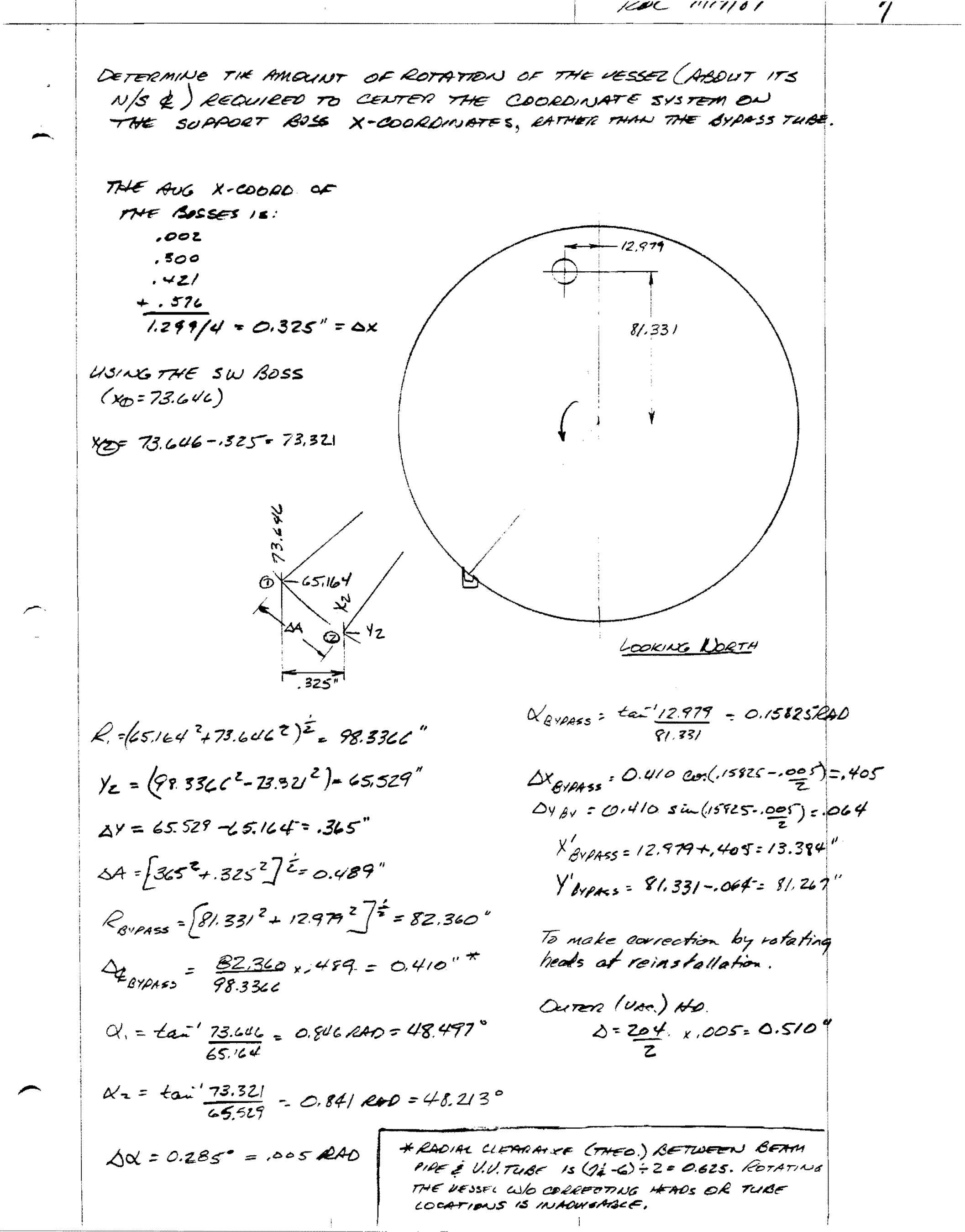




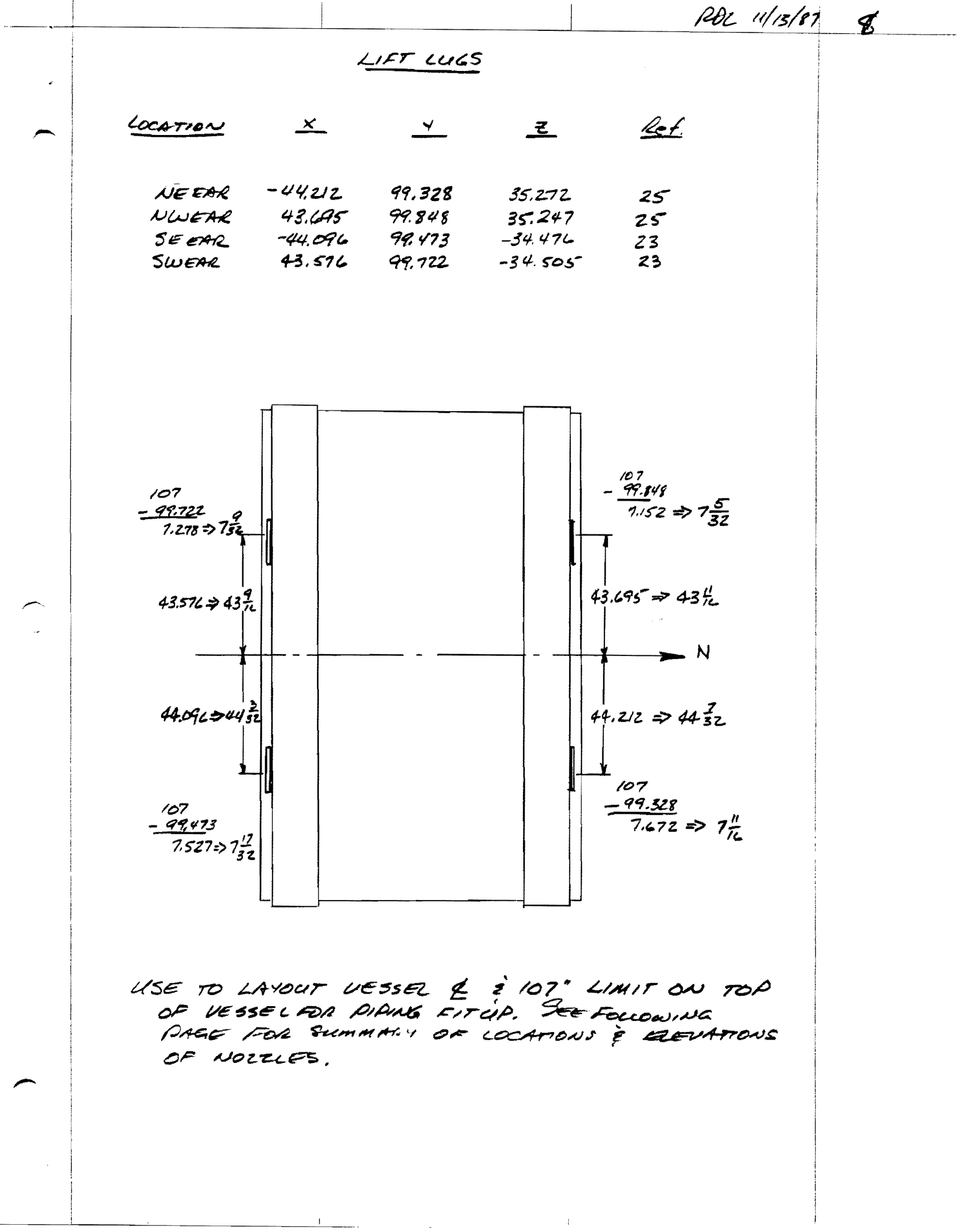


100

TD, CW,MC HORZ, OISTANCES TO NOZZLES PIPS SUPROKTS 12.5.8?. AANO, REF BARS LOCATEO ON ITOP OF VESSLL OK $0.0,75-1$ ACCURACT IS \pm 100

NOTE: ALL MEASUREMENTS ARE, FROM

A. LINE OEFNEO BD EXISTINE

NORTA

PUNCN MARKS ON TOP OF VESSEL :

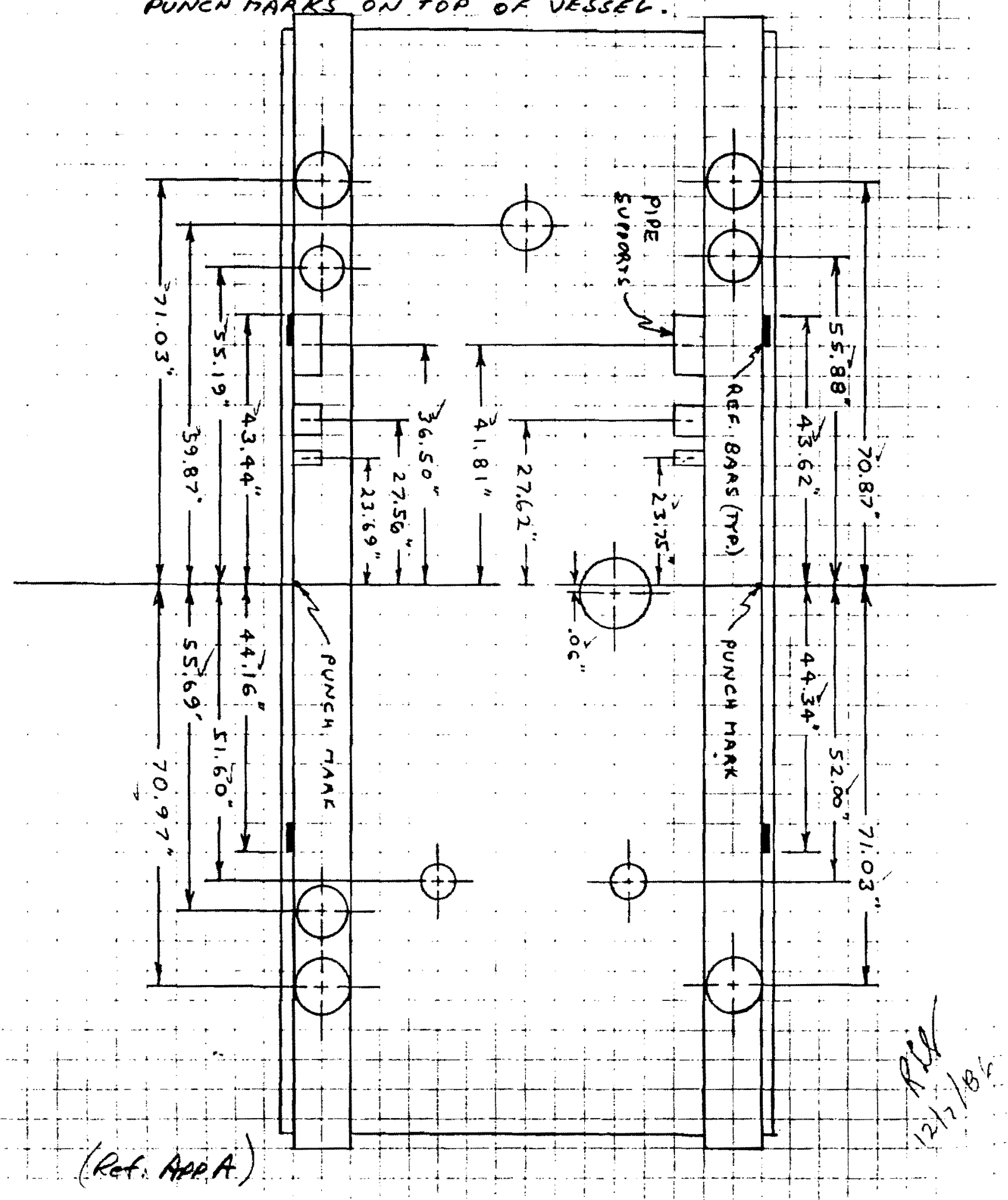




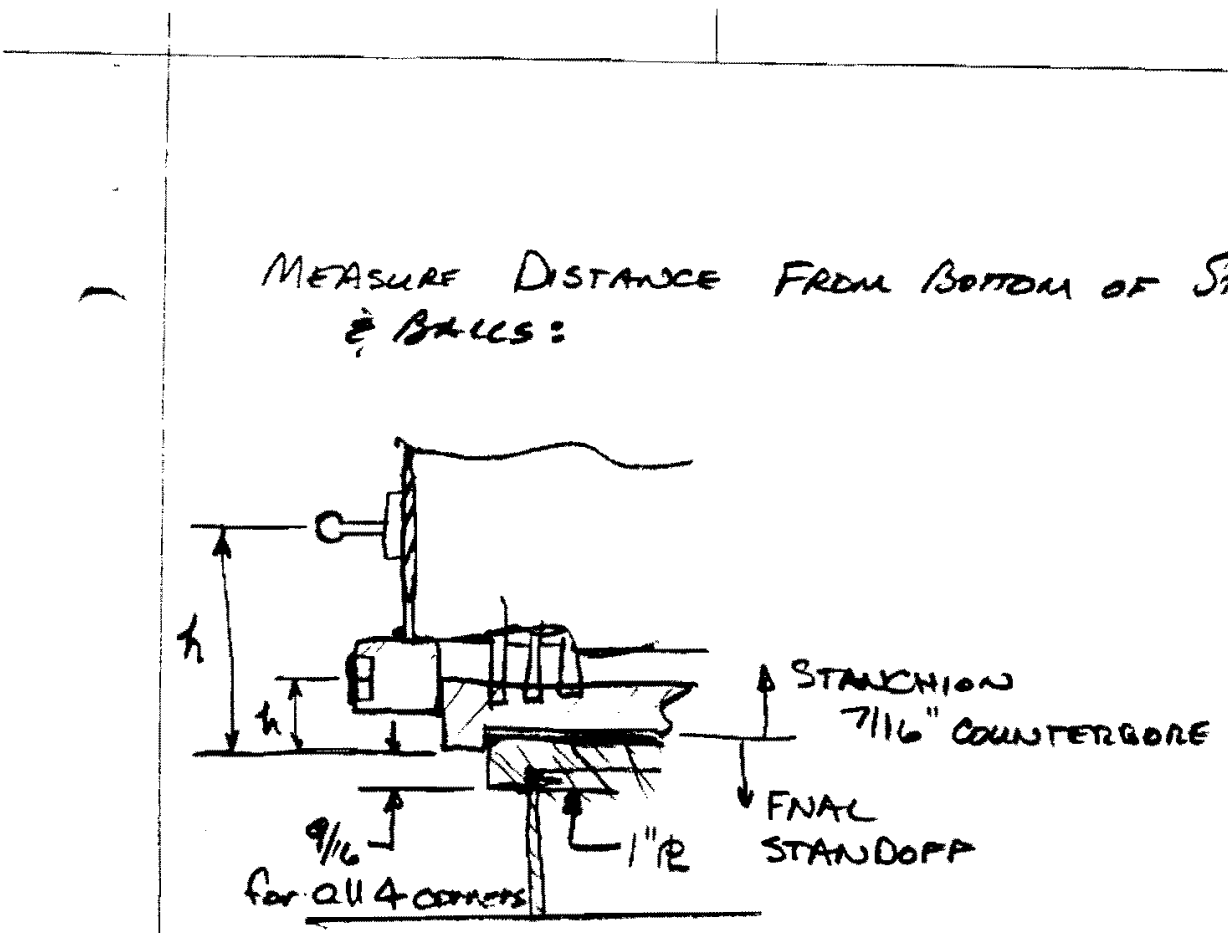

12

ReC $11 / 13 / 87$

Asure Distance From Borrom of Stamcition to taxeets Exices:

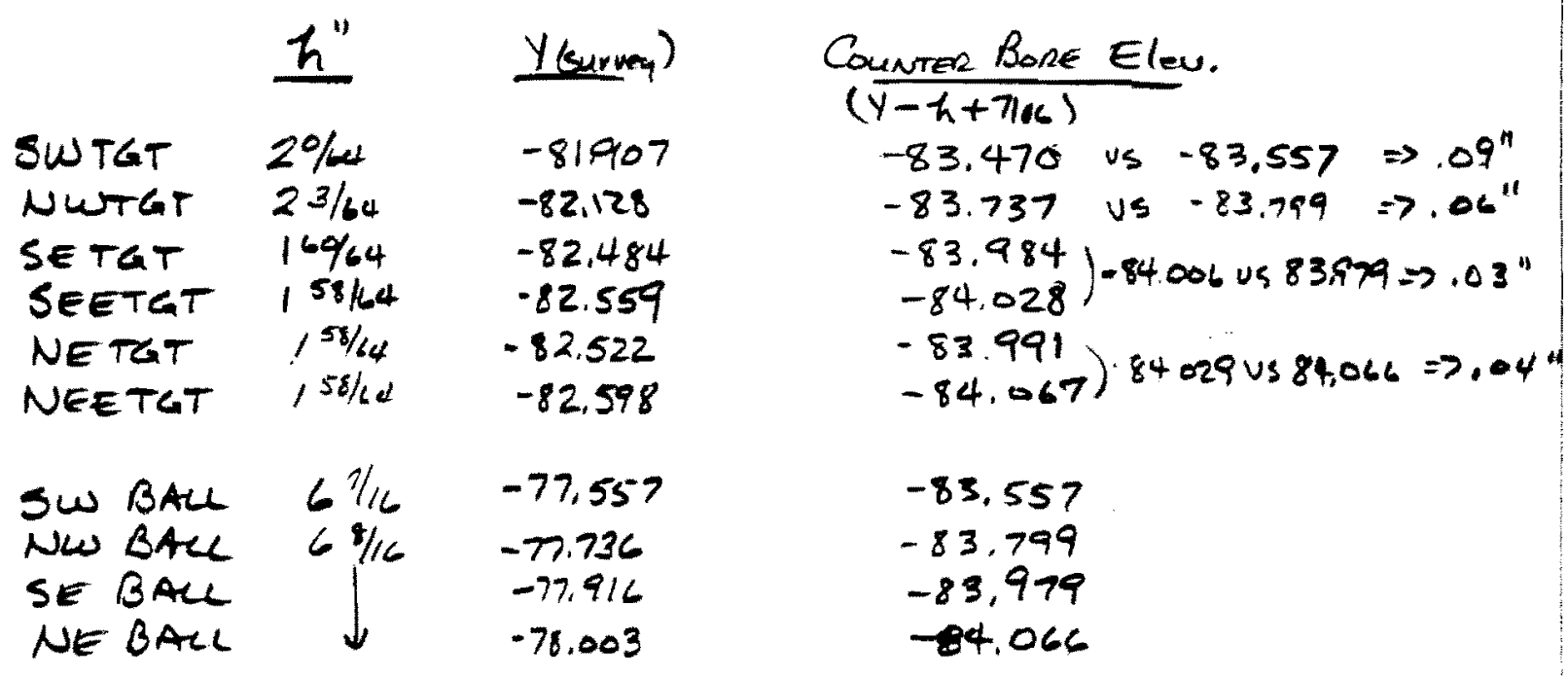

USE TGT MEXSUREMENTS:

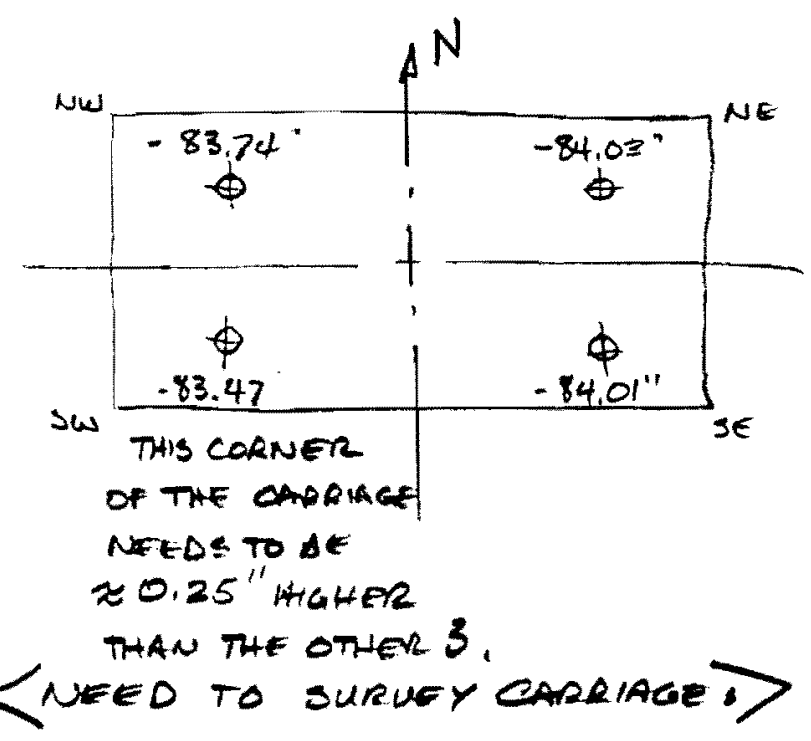


$I D, C \omega_{,}, M C$ $12-2-87$

BK $0,0,75-1$
SUMMART.

of

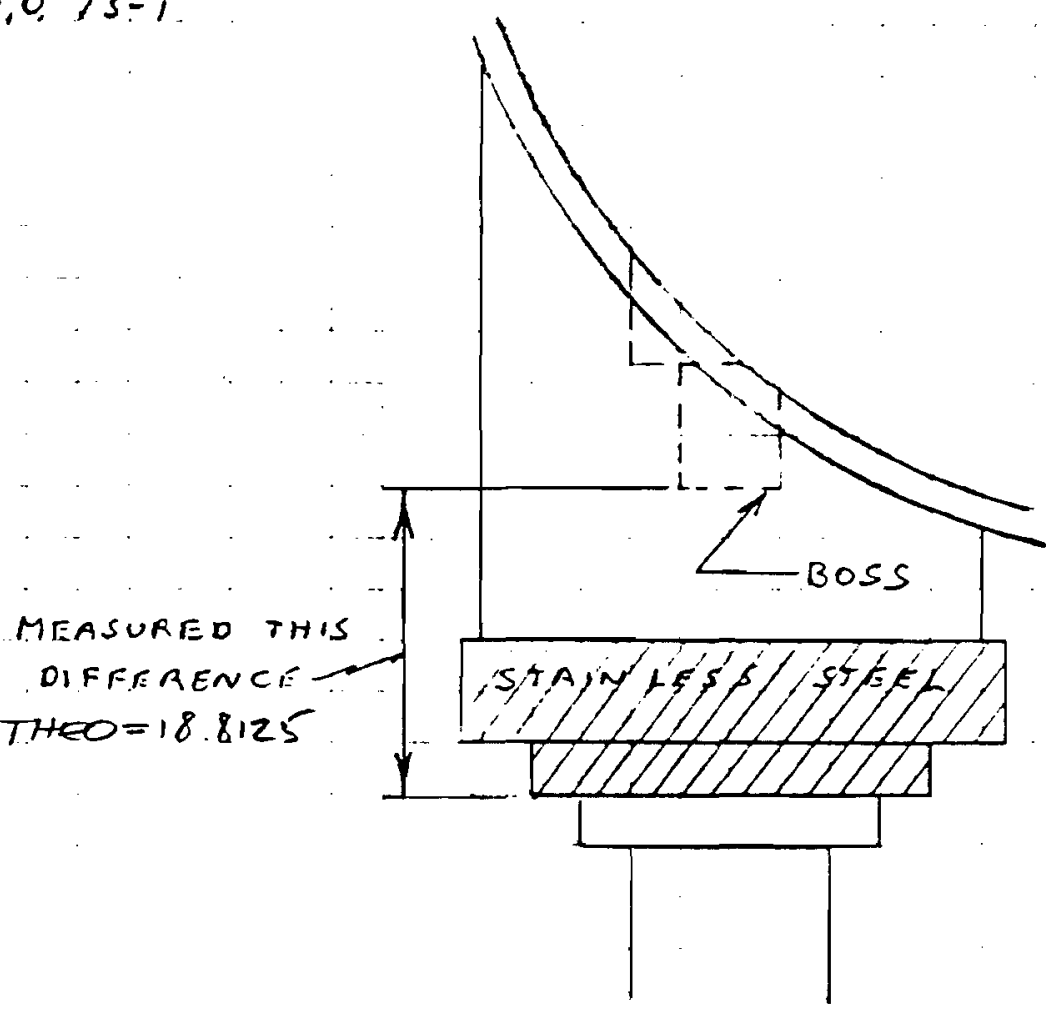

\begin{tabular}{|c|c|c|c|}
\hline LOC & CATION & O,FEERNCE & GAP $(=T H E D-M E A S U R F C)$ \\
\hline N.E. & e.s 5 & $18.913^{\circ}$ & $0.100^{\prime \prime}$ \\
\hline$\sim w$ & coss & $18.918^{\prime \prime}$ & 0.105 \\
\hline$S E$ & $B \circ 55$ & $18.892 "$ & 0.079 \\
\hline sw & $B O 5 S$ & $18.898 "$ & 0.035 \\
\hline
\end{tabular}

Results indicate that 1, 2, or 3 of the stanchions may zeot be completely compressed. Since the presence of gops in the stenchions arill complicart the esseubly of the motules, a. methof of determining and eliminationg any gaps will be. developed. Since looth Worth end stacks appent to have gapsp it is suspocted that the forces exerted by the bellows. C $\mathrm{k}, 81$ on top of the resser are sufficient to move the wessel 2,3 when the heads are remoced. (Nate: Fus stanchions. IV \&D were tight when installed. The weight of the heaks is affroximetely $2 / 3$ of the total wh of the eryostet.) 


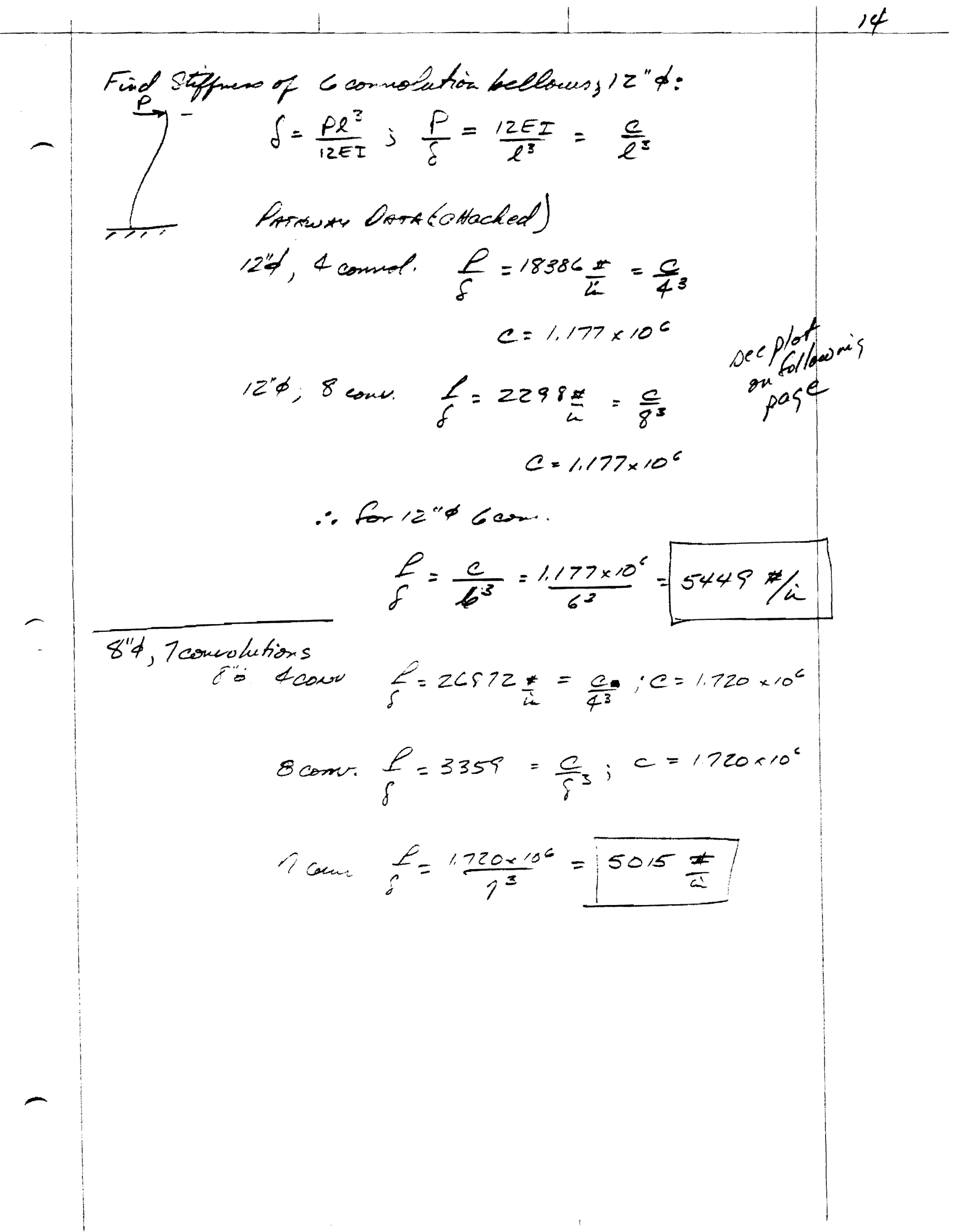



NG

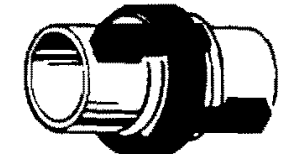

NH HINGE

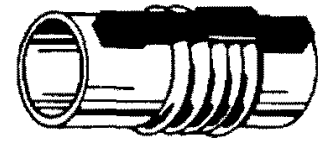

NT

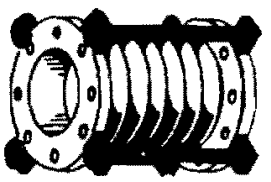

NS

SINGLE

UNRESTRAINED

EXPANSION JOINT

Pressure Thrust equals 151.2 sq. in. times Pressure

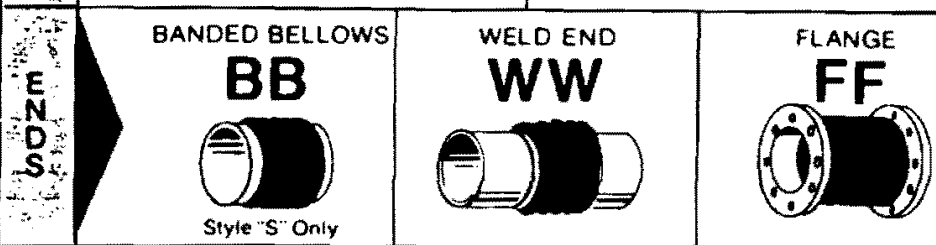

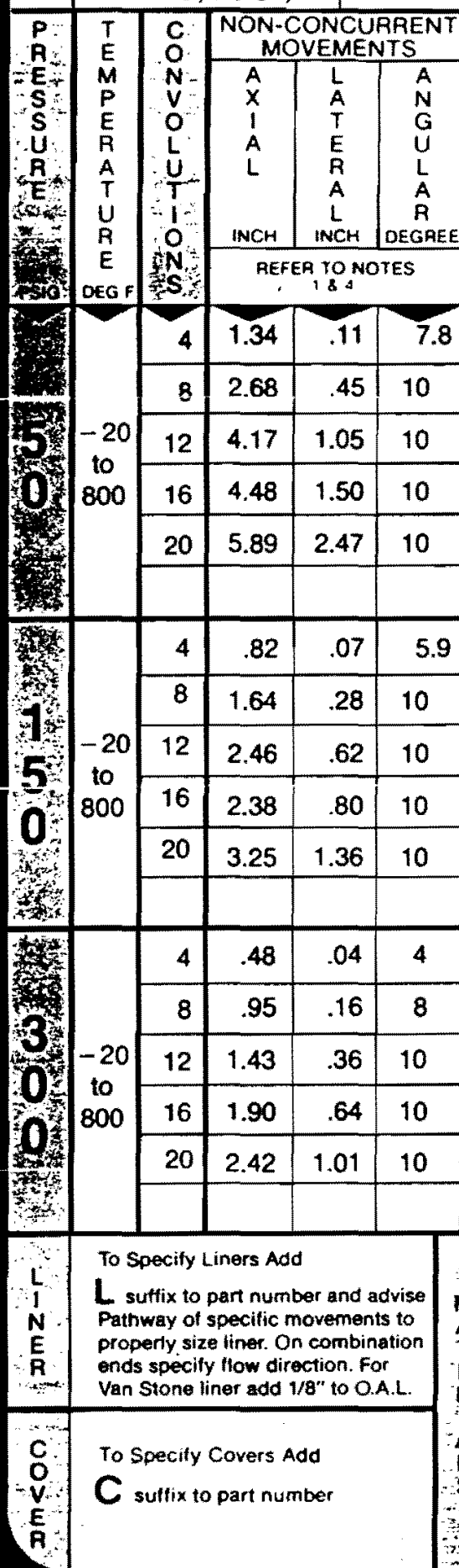

\begin{tabular}{l}
\hline \\
\hline$x$ \\
1 \\
$A$ \\
$L$
\end{tabular}

SPRING RATE

VAN STONE FLANGE VAN STONE/WELDEND FLANGE/WELD END

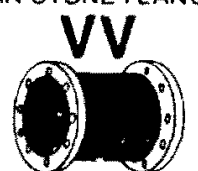

16 Con Maximum

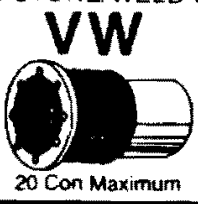

EVI

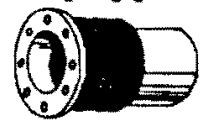

OVERALL LENGTH AND WEIGHT

\begin{tabular}{|c|c|c|c|}
\hline \multicolumn{4}{|c|}{ ADD TO THE LENGTH BELOW } \\
\hline $\begin{array}{l}\text { O.A.L. and } \\
\text { weights beiow } \\
\text { are lor Style } S\end{array}$ & $\begin{array}{l}\text { Stre "G" } \\
\text { W-W } 8 \text { in. } \\
\text { WW } 12 \text { in. }\end{array}$ & $\begin{array}{l}\text { sope } 7 \\
F W-W=8 \text { in } \\
W W 12 \pi \text {. }\end{array}$ & $\begin{array}{l}\text { Style T } \\
\text { FW-WW } 7 n \\
\text { Ww } 10 \text { in. }\end{array}$ \\
\hline
\end{tabular}

WORKING WOAKING WOAKING \begin{tabular}{l|l|l} 
LOS/NCH & WOAKING & WOAKING \\
\hline LAS/INCH & IN LBS/OEG \\
\hline
\end{tabular}

\begin{tabular}{l|l|l|l} 
BB & WW & FFNV & FW/WW \\
\hline
\end{tabular}

563

18386

236

\begin{tabular}{|l|l|l|l|l|l|l|l}
\hline O.A.L. & WT. & O.A.L. & WT. & O.A.L. & WT. & O.A.L. & WT. \\
\hline
\end{tabular}

\begin{tabular}{l|l|l}
282 & 2298 & 118
\end{tabular}

\begin{tabular}{|c|c|c|c|c|c|}
\hline $91 / 2$ & 9 & 13 & 43 & $113 / / n$ & 137 \\
\hline
\end{tabular}

188

$681 \quad 79$

\begin{tabular}{|l|l|l|}
\hline 13 & 13 & $16 \frac{1}{2}$ \\
\hline
\end{tabular}

\begin{tabular}{l|l|l|l|l}
47 & $14 \%$ & 140 & $151 / 16$ & 72 \\
\hline
\end{tabular}

275

$562 \quad 116$

220

288

92

\begin{tabular}{|l|l|l|l|l|l|l|}
\hline $16^{1 / 2}$ & 16 & 20 & 50 & $183 / 8$ & 144 & $19 \%$ \\
\hline 20 & 20 & $231 / 2$ & 54 & $21 \% / 8$ & 147 & 221 \\
\hline
\end{tabular}

79 


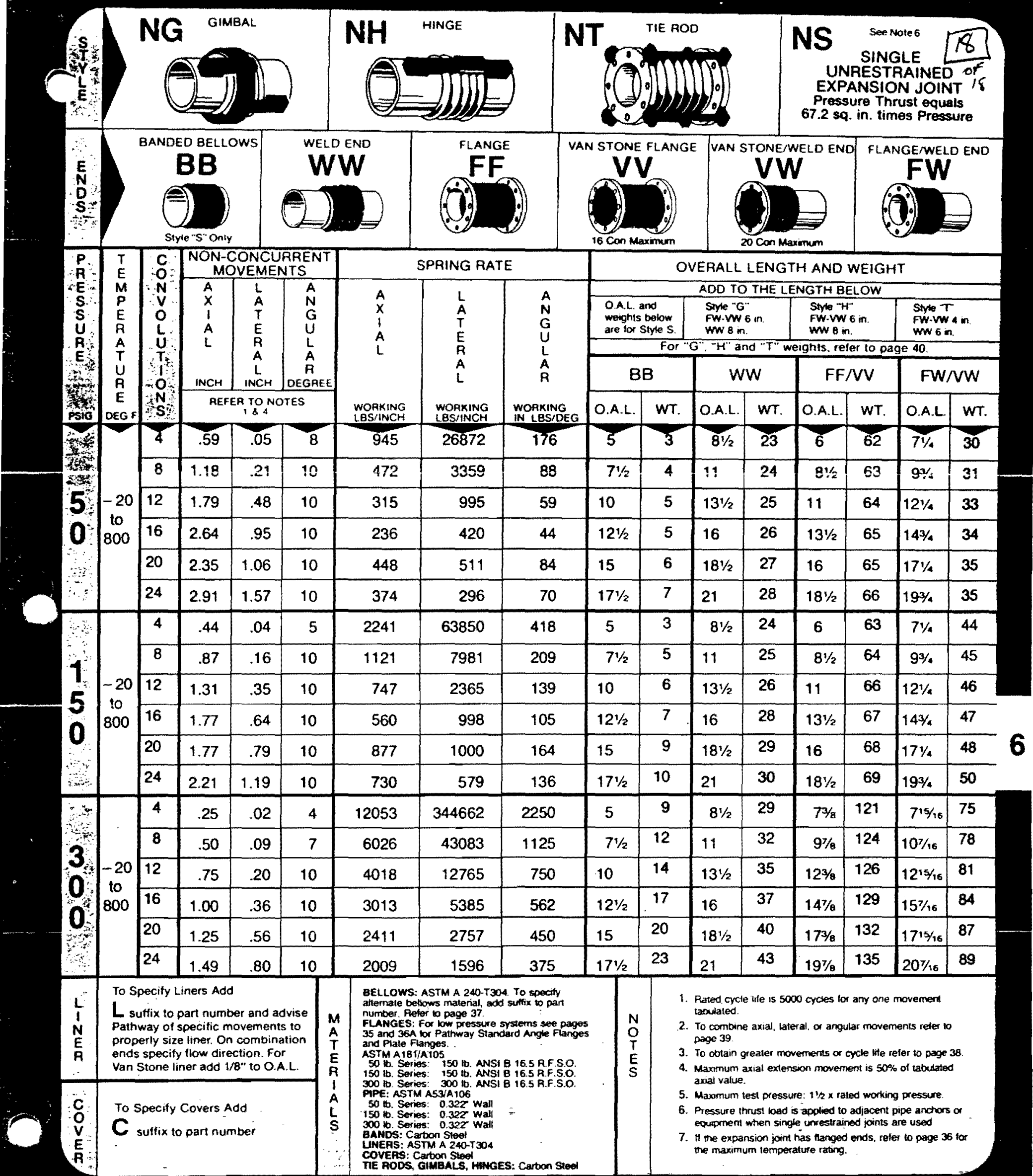




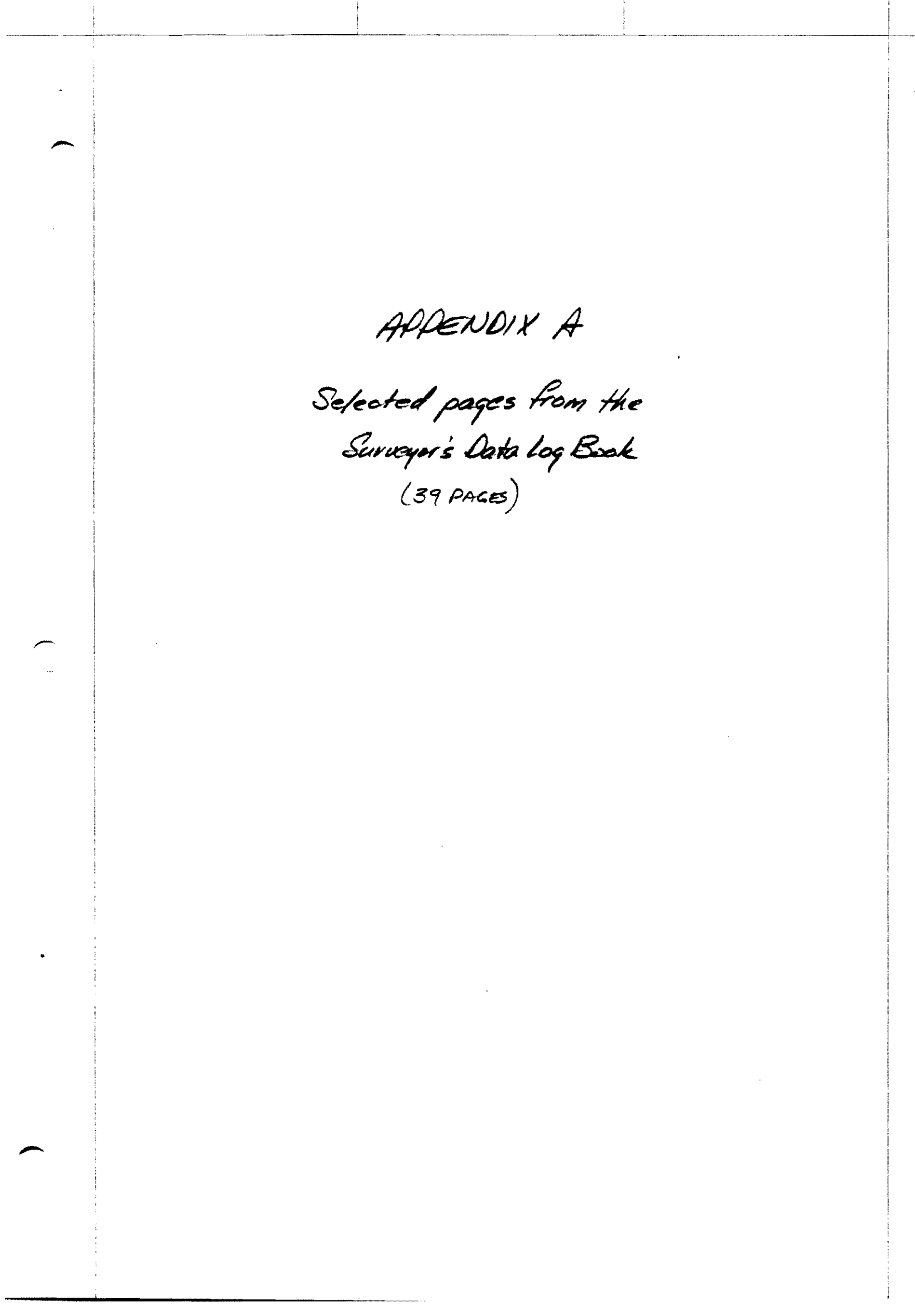


Jo, CW DO DETECTOR ENO CAP REEFRENGE LO-12-87 LOCATION OE POINTS FuAVEREO BK DO 25-1 SES BETS FKE ROR COORRUATES $P$ 10-26, 7HKS BK
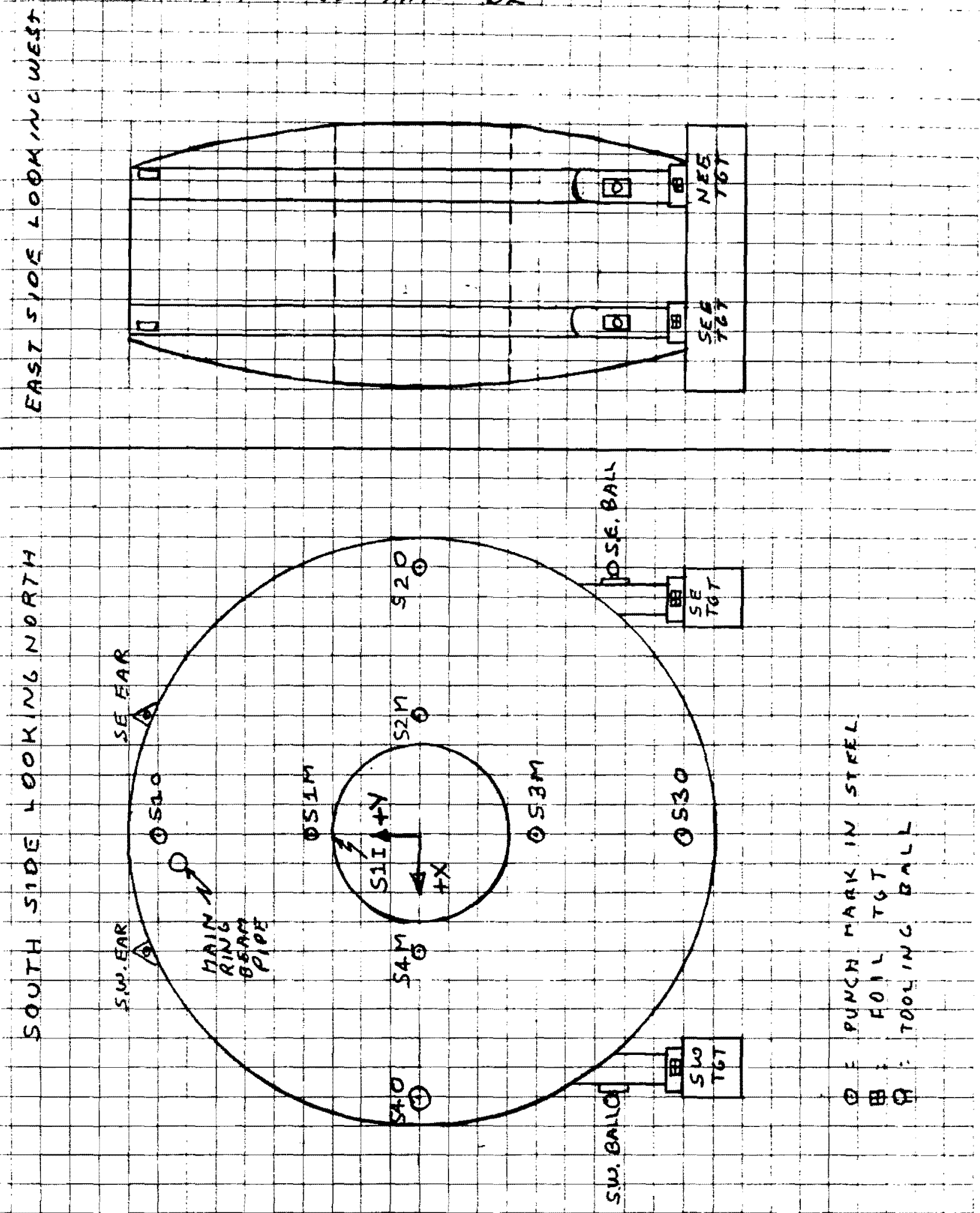
8

JD.CW

$10-09 \cdot 8)$

BK DO $75:-4$
IT: WAS OECHOEO BY RICK LUMOER TOH REFERENCE THE OIO DETECFOR LORE USING THE L OE THE TMSTOE LHLANAR AS THE HORZ T HERX \& I TME FOEA 4

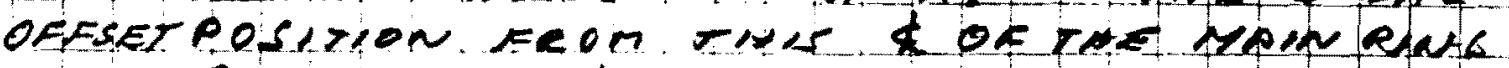
QEAM: PUPS IS TO BE USEO FOR RPTATON

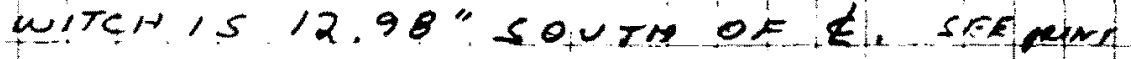
$3240+214-1,19-2432=$

LOOKING NORTIX

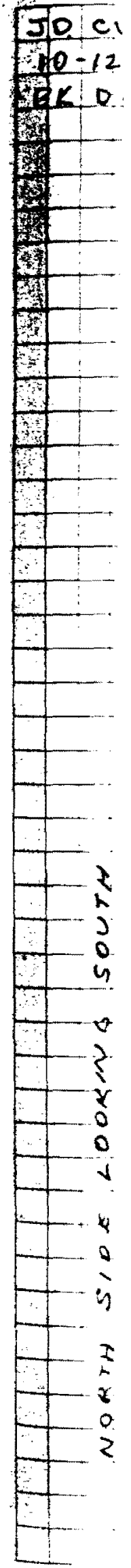

SET UP MICROMERERS. IN ESNTER OF DONUTS TO OERNE L ANO ORIENTATE OFE OF WITH BLTSL

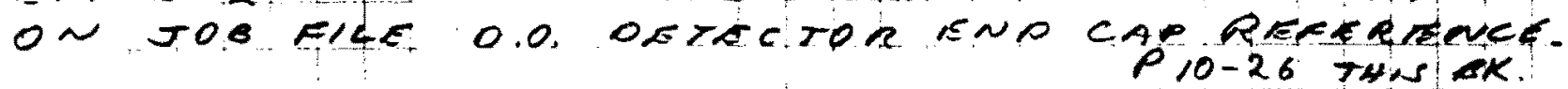
MICROMETER READNCS

\section{SOUTH ENO}

$0.488 " .495^{\circ}$ $45^{\circ} .5533^{\circ} .555^{\circ}$ $90^{\circ} .3,26 . .355^{\prime \prime}$ $135^{\circ} .480^{\circ} .503^{\circ} .170$ $180^{\circ} .484^{\circ} .485^{\circ}$ $225^{\circ}, 398^{\prime \prime} \quad$ i376" $270^{\circ} .4 \times 8^{\circ} .381^{\prime \prime}$ $3.5^{\circ} .372 . .270^{n}$
NORTN IENO

$0^{\circ}, 482$

$45^{\circ} .350$

$90^{\circ} .260^{\prime \prime}$

$135^{\circ}, 460$

$180^{\circ} \quad 480$

$225^{\circ} \quad 465^{\circ}$

$270^{\circ}: 260$

$315^{\circ} .470^{\circ}$ 
$A-4$

JD.

$\checkmark 0-13-87$

$B K \quad 00,75-1$
SUMMART OF TOOLINC BALL AND FOL TARLET COONOINATES OF TWE D O OETECTOR CORE TROM, T-26 THIS BK.

NOTE: THERE WILL BE A - 48.466"Z CORECTION ON THESE COOT OINATRS 50 TMA I EVERTIHMG IS RELATIVE TO A $X, Y, Z$ L OF CORE. $Z \notin$ IS DETERMINTO' $B$ V THE MID DONT BETWEEN THE SUPPORT NOZZLFS. AS PER PRINT \# $3740-214 \cdot M E-2232$ BS SHT 1

PLAN VIEN YISHEIGHT

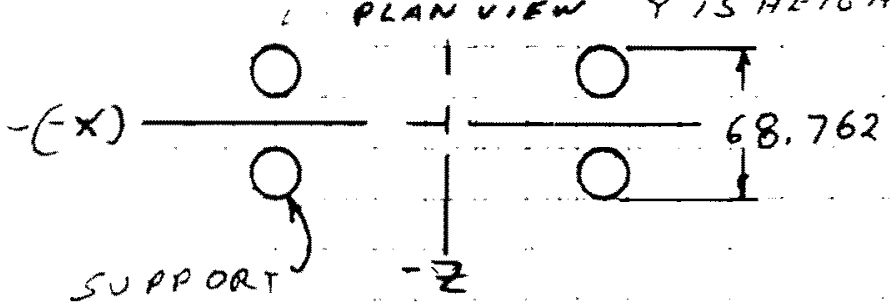

MOZZLAS

$$
\begin{aligned}
& \begin{array}{c}
5 \omega .765 \\
-x-73.516
\end{array} \\
& r-8 \% .907^{\circ} \\
& z-34,229^{\prime \prime} \\
& \text { SETCF }
\end{aligned}
$$

NW BALC

$$
\begin{aligned}
& -x-81.114^{\prime \prime} \\
& Y-77.736^{\circ} \\
& \text { z 28.205" } \\
& \begin{array}{l}
\text { NE BALL. } \\
-{ }^{2} \text { B0.804" }
\end{array} \\
& r-78.003^{\prime \prime} \\
& \text { z } 27.476^{\circ}
\end{aligned}
$$$$
-x>2,7>7 .
$$$$
r=82,484^{\circ}
$$$$
z-34,534 \text {. }
$$

$$
\begin{aligned}
& \text { NWTOT } \\
& -x-73.202 \\
& y-82.128^{\circ} \\
& \text { z } 34.531 \text {. } \\
& \text { NETGT } \\
& -x>2778^{\circ} \\
& \text { Y }-82.522 . \\
& \text { z 3.4.230" } \\
& \text { NEETUT } \\
& \text { - } X>9.713 \\
& r-82.598^{\circ} \\
& \text { z. } 27.395^{\circ} \\
& S E R T \\
& -x \quad 79,534 \\
& \gamma \quad-82,559^{\circ} \\
& z \quad 27864^{\prime \prime}
\end{aligned}
$$$$
\text { SW BALL }
$$$$
-x \quad-81.478^{\prime \prime}
$$$$
S E B A L 6
$$$$
\text { - } \times \quad 80.594^{\circ}
$$ 
A.5 7

T.D. RS $11-0 \leq-87$

L $1 \%$ D $75-1$
LAB.A.. D.O. CENTRAL DETELTOR

SUAUET OF ARGON VESSEL SUPPOTT BOSSES SEE BETS HLE [D-O CENTRAL OTT INNER, SUPPORT] $P 78-81$ THIS. BK.

PLAN VIEW

NOTE: ALL COOROINATES ARE RELATUF TO ORIENTATION SMEWN ON P.8 TNIS OK.

$Z$ = HEICNT DIER RROM CENTH

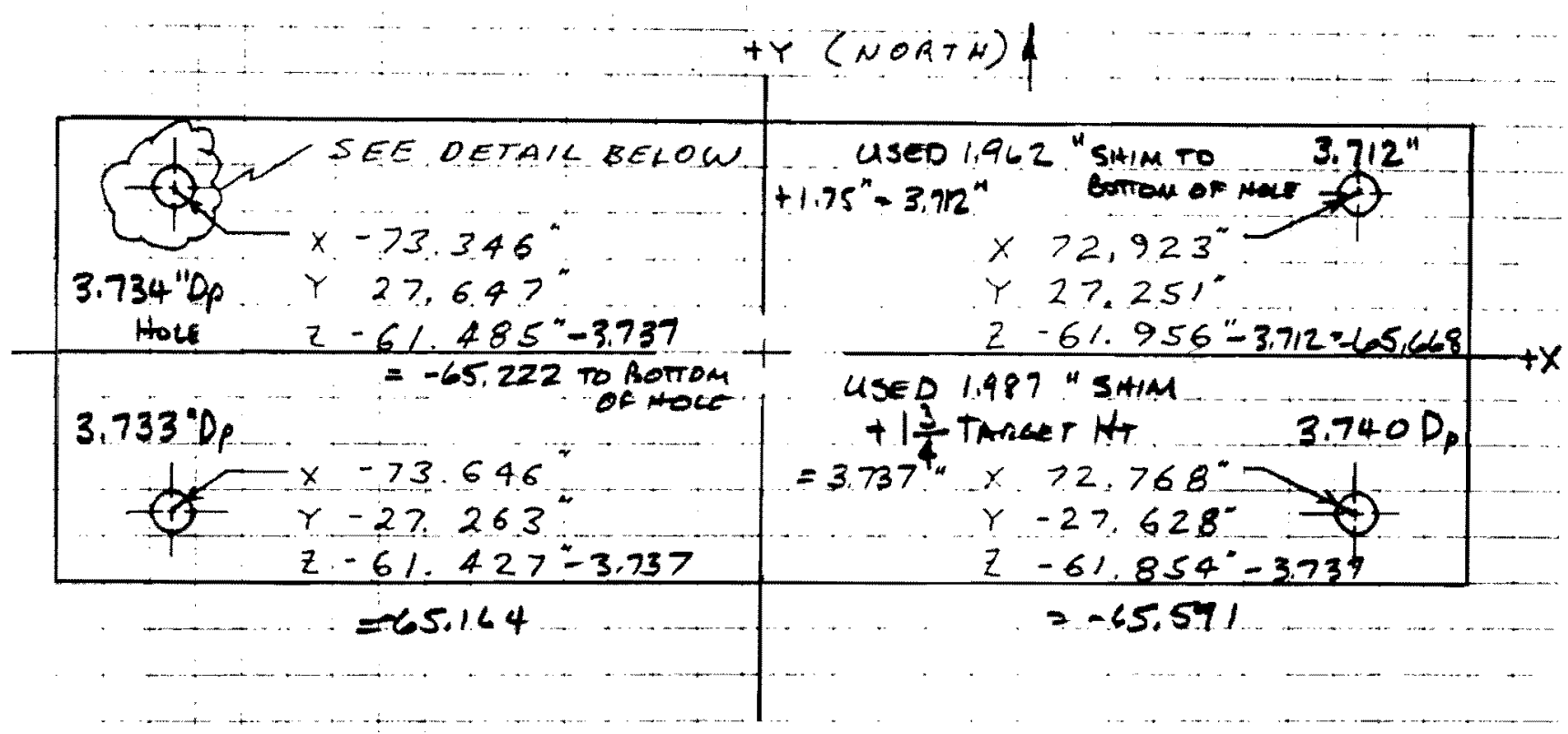

FRONT VIEW OR BOSS

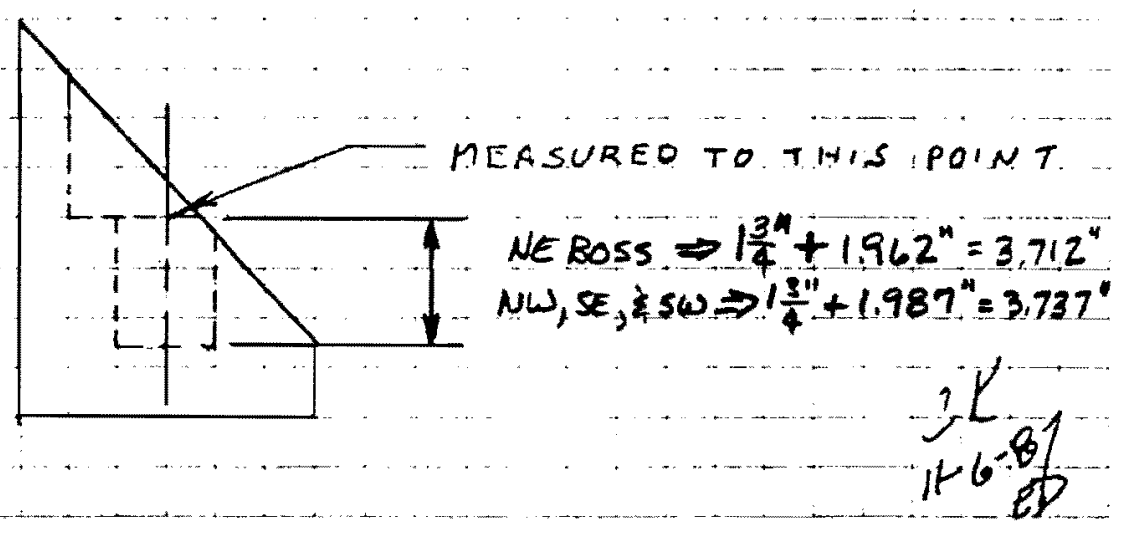


$n$

75

JORS

$11: 5: 87$

AK OO. . $05-1$

PLANE: ANALY SIS FOR BOSSES SURVEFSO ON R.T THIS BOOK

$11 / 05 / 87$

$10: 12$

JOB FILE: D- $\emptyset$ CENTRAL DET. INNER SUPPORT SURUEY

OPERATORS: JAMES DAHLBERG, RICH SMITH

LOCATION: LAB A

MEASUREMENT UNITS: Inches

COMMENTS:

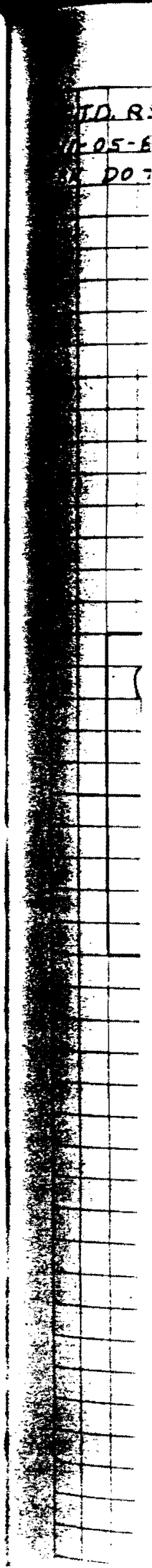

POINT

WORST FIT

RMS FIT

0.0112 Inches

0.0112 Inches

WORST FIT POINT NEBOSS

SWBOSS

FIT

POINT

FIT

POINT

FIT

SEBOSS

0.0112

NWBOSS

0.0112

NEBOSS

0.0112

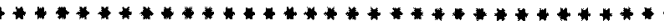

***** END OF PLANES ANALYSIS - D-O CENTRAL DET. INNER SUPPORT SURUEY ***** 


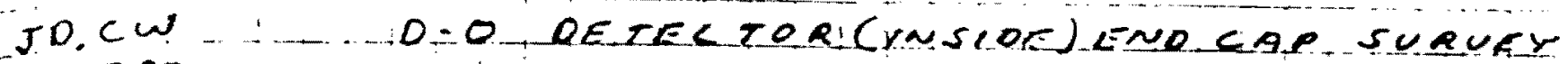
$10-23-8 ?$ $6 \times 0.0,75-1$

SUMMART OF-SURUEL POVNTS PERTRENCEO OKRING BETS TOBIFLE (SAMF AS TITLA AOOW) SOUTH SIOE (INSIOE) ENOCAP. LOPKING NORTH

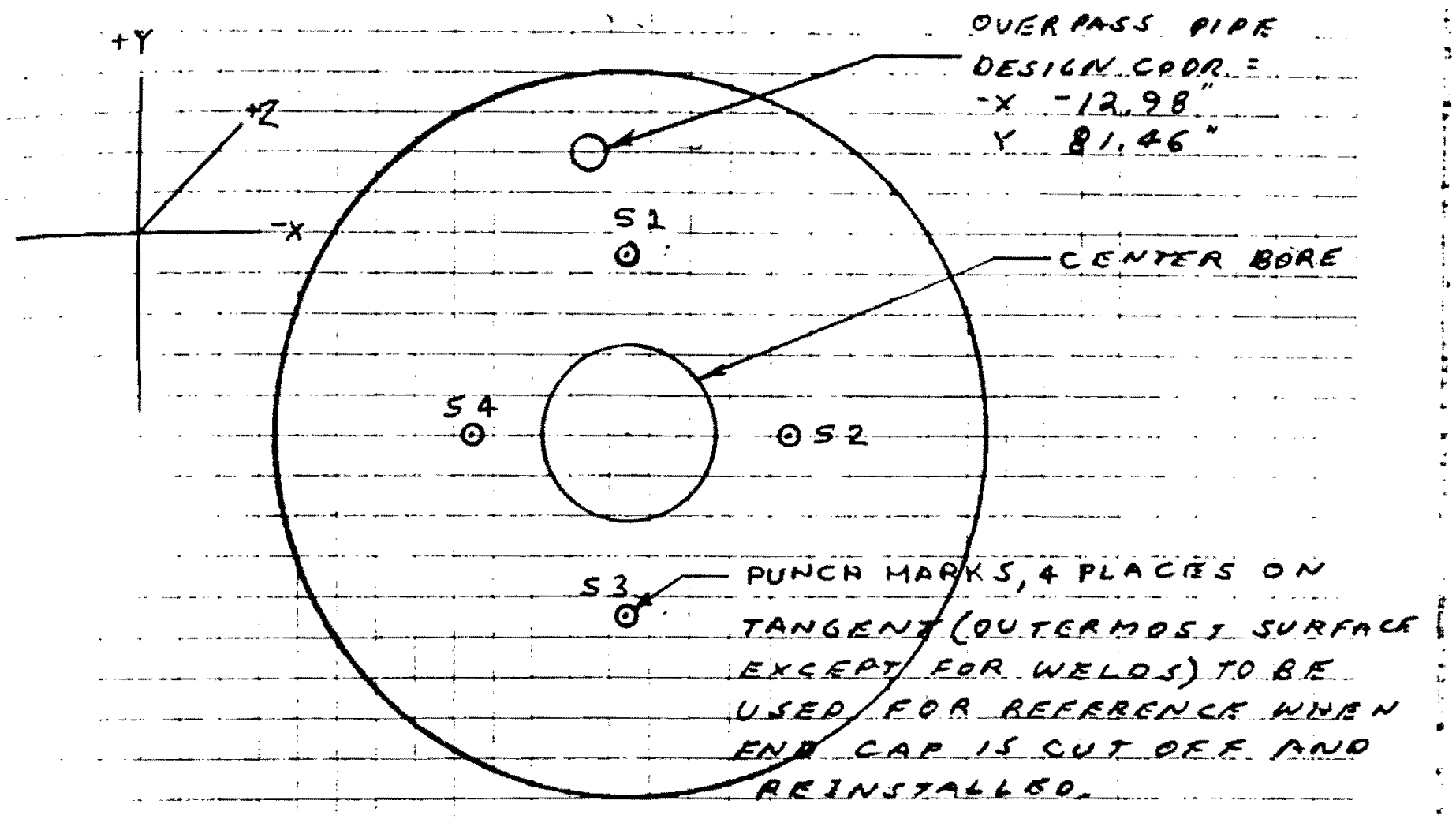

COORDINATES OA SUAUEP PONNS ABOUE. RELAYILE TO ORIENTATION WSEO ON P.B THIS BOOK.

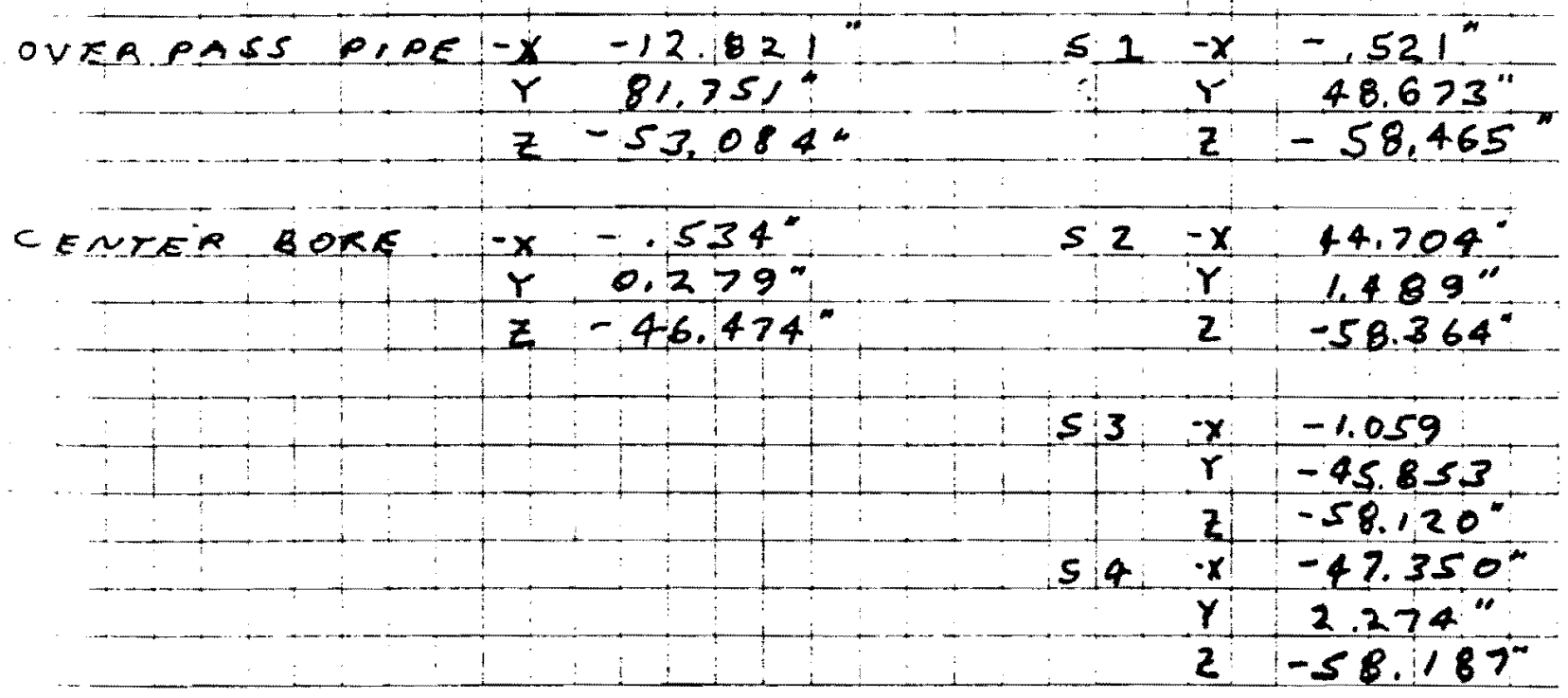



$10-23-8)$

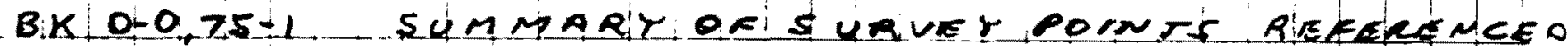

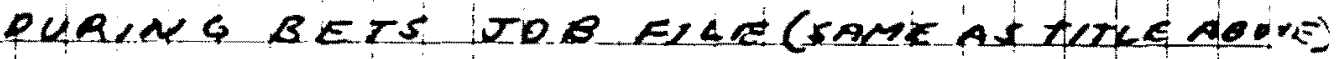

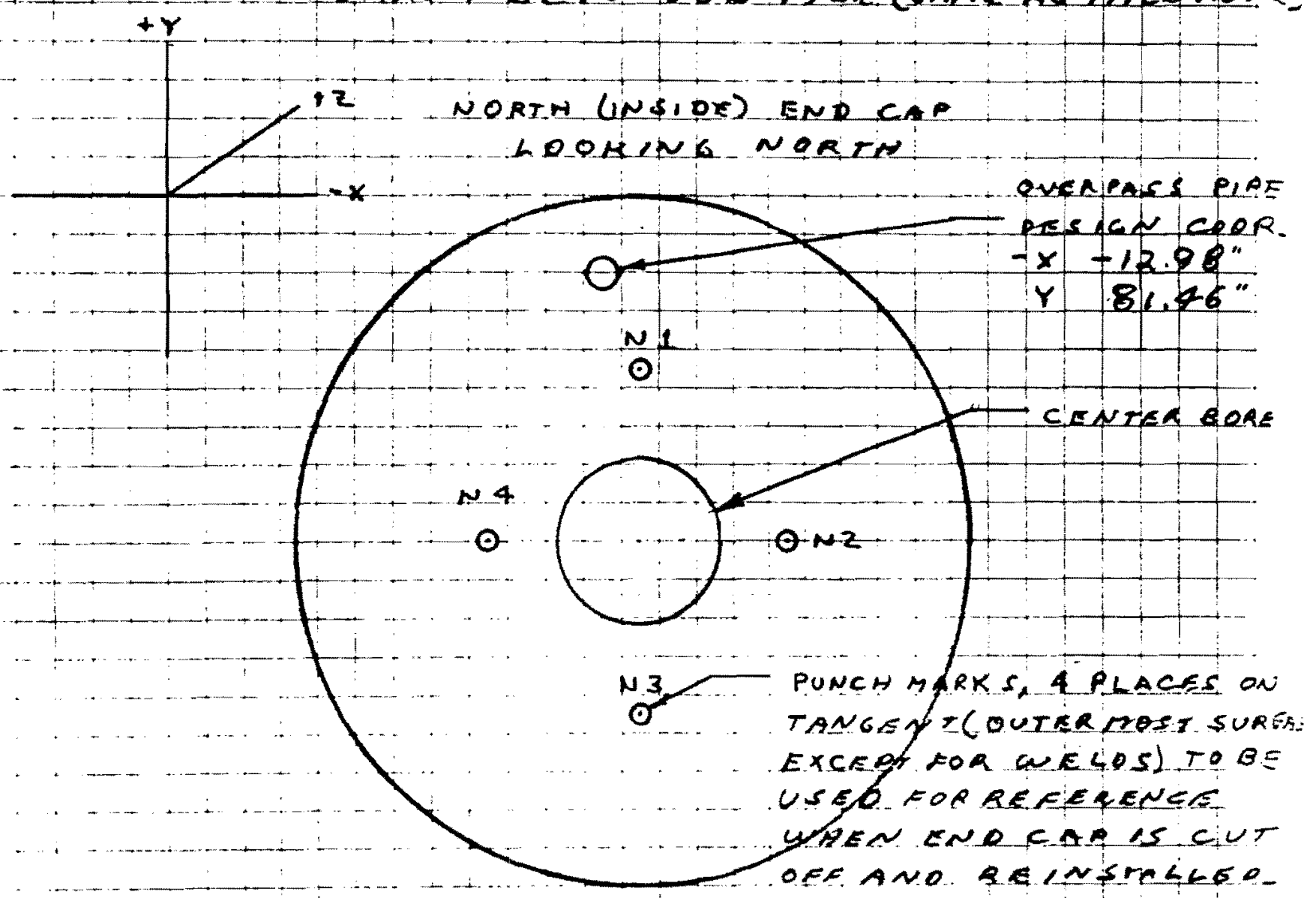

COORDINATES OR SURUEU CPINTS ABOUE RELATINE TO ORIATTATIAN USED ON P. B THIS BOCR.

ovepenss pies it -12926"

r. $81,475^{\circ}$

z $\quad 53.661^{\circ}$

CENTER

core

$\begin{array}{cc}-x & -0.790^{\circ} \\ Y & 0.223^{\circ} \\ z & 46.463\end{array}$

-x. $1.060^{\circ}$

Y 49.173

z. 58.605

N 2
N. 2 - 48,235

$r \quad 0.415$

$z \quad 58,470$

$\sim 3 \quad-x-1496$

$Y \quad-46.822$

2. 58.868"

$N-4 \quad-x-46,262$

$r \quad 0.182$

z. $58>35$

$2+58,60$ 
$10 / 22 / 87$

$21: 38$
FERMILAB SURUEY/ALIGNMENT
$J O E R$ R C AP REPORT

\author{
JOB FILE: D-D DETECTOR INSIDE END CAP REFERENCE \\ OPERATORS: CHARLES WILSON, JIM OAHLBERG \\ LOCATION: NWB-LAB A \\ MEASUREMENT UNITS: Inches \\ COMMENTS: REFERENCE INNER END CAPS AFTER OUTER END \\ CAPS WERE REMOUED.
}

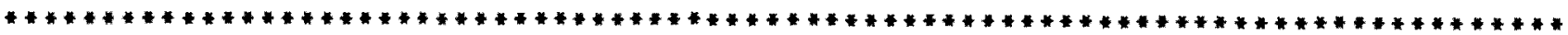

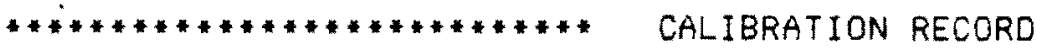

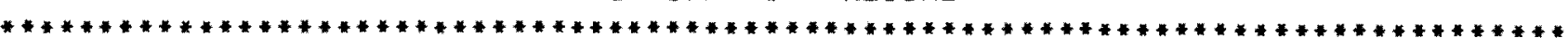

CALIBRATION NAME: TCSOI

CALIBRATION TYPE: New Calibration

CALIBRATION LOGGED: 10/21/87 - 19:07

CALIBRATION BAR LENGTH: 114.9999 Inches

REUERSE AND PLUNGE?: N

\# OF SHOTS:

CALIBRATION INDEX: .3

THEODOLITE BASELINE LENGTH: 182.1924 Inches

THEODOLITE HEIGHT DIFFERENCE: $\quad-27.2382$ Inches

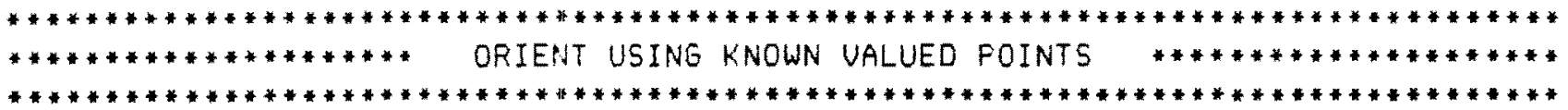

$$
\begin{aligned}
& \text { ORIENTATION NAME : SOUTH } \\
& \text { REFERENCE POINT UALUES: Manually Entered } \\
& \text { ORIENTATION LOGGED: } 10 / 21 / 87-19: 27 \\
& \text { * * Reference point values * * * }
\end{aligned}
$$

$\begin{array}{rrr}-X & 2 & y \\ -73.5160 & -34.2290 & -81.9070 \\ 72.7770 & -34.5340 & -82.4840 \\ 80.8040 & 27.4760 & -78.0030 \\ * \text { * POINT FIT DATA * * * }\end{array}$


$10 / 22 / 87$

$21: 38$
FERMILAB SURUEY/ALIGNMENT

$J O B$ RECAP REPORT

Page 2

JOE FILE: D-e DETECTOR INSIDE END CAP REFERENCE (cont'd)

CALIBRATION NAME: TCSO2

CALIBRATION TYPE: New Calibration

CALIERATION LOGGED: $10 / 22 / 87-18: 37$

CALIBRATION BAR LENGTH: 114.9999 Inches

REUERSE AND PLUNGE?: N

\# OF SHOTS: I

CALIBRATION INDEX: 2.1

THEODOLITE BASELINE LENGTH: 109.5960 Inches

THEOCOLITE HEIGHT OIFFERENCE: 3.4948 inches

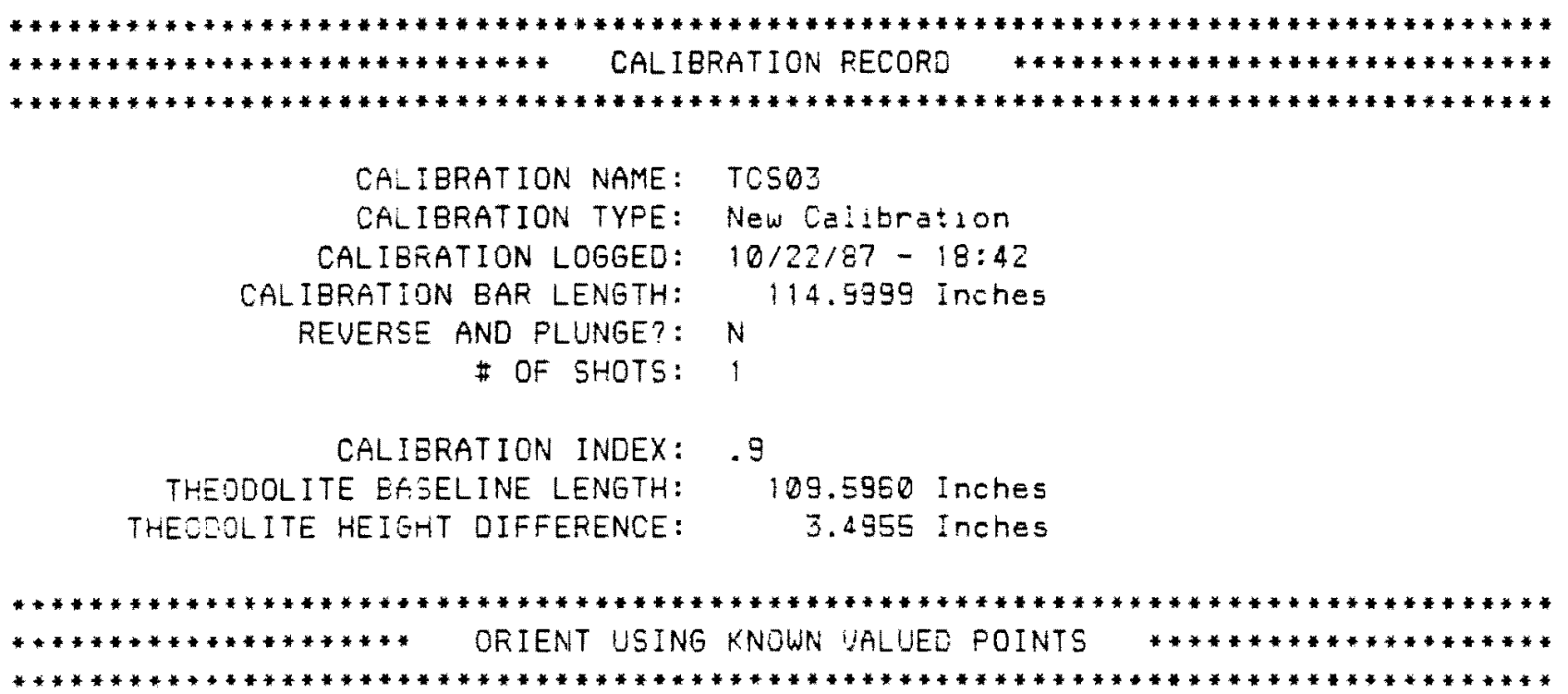


$10 / 22 / 87$

$21: 38$
FERMILAB SURUEY/ALIGNMENT

$J O B$ RECAP REPORT
Fage 3

JOB FILE: D-O DETECTOR INSIDE END CAP REFERENCE (cont' $d$ )

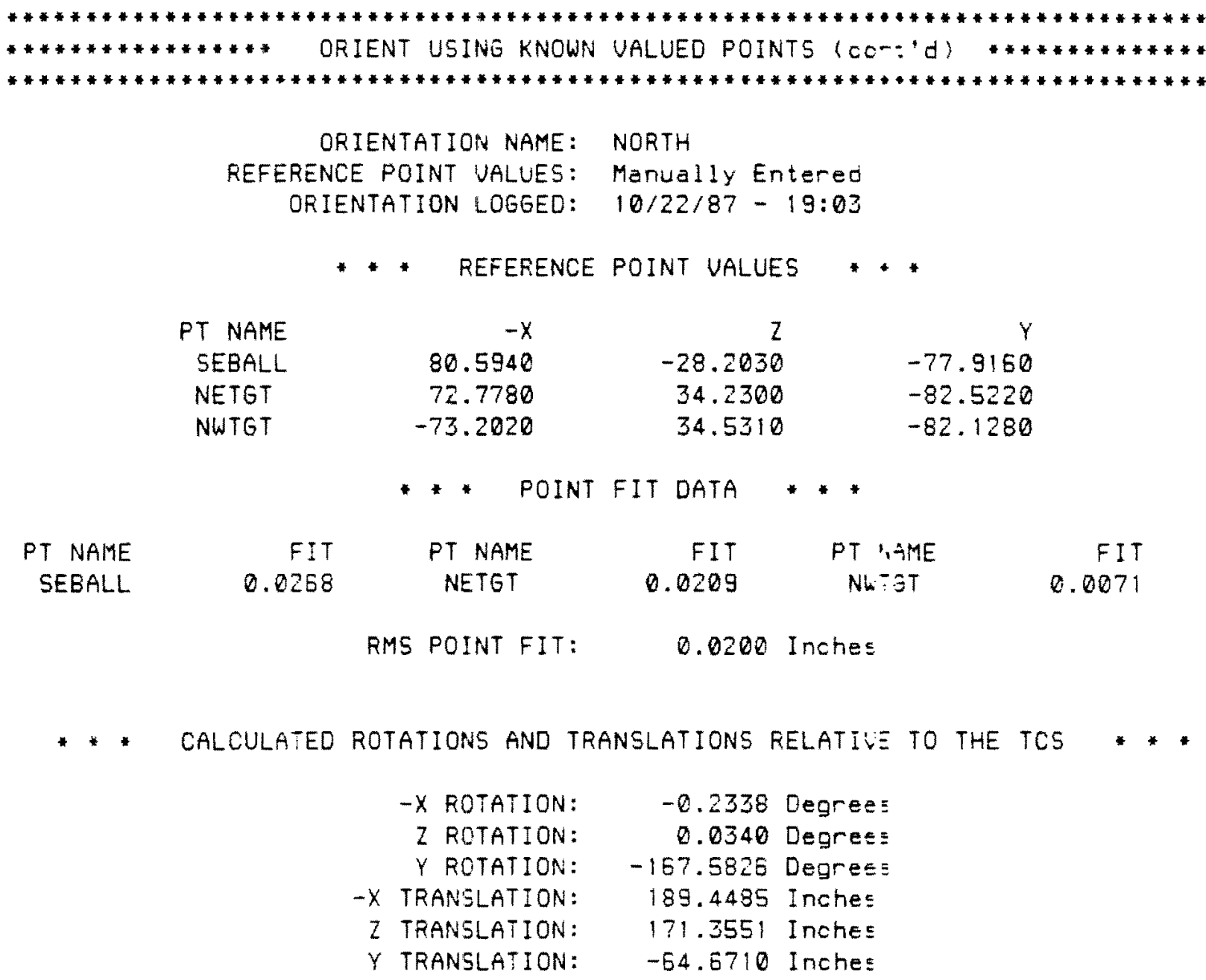

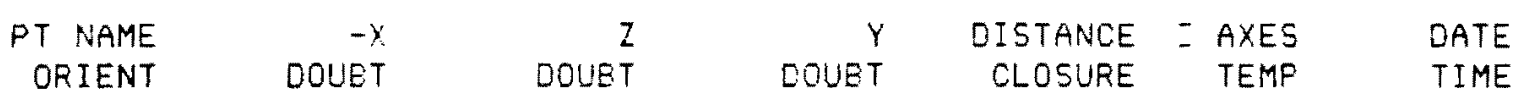

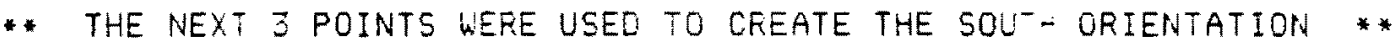

REFERENCE UALUE POINT NAME FOR THE FOLLOWING POINT WAS: SW-TGT

$\begin{array}{rrrrrrr}000001 & -73.5205 & -34.2355 & -81.9078 & 0.0000 & --2-Y & 10 / 21 / 87 \\ \text { SOUTH } & 0.0015 & 0.0017 & 0.0008 & 0.0030 & 70 F & 19: 15\end{array}$

FEFERENCE VALUE POINT NAME FOR THE FOLLOWING POIN" WAS: SE-TGT 
$10 / 22 / 87$

$21: 38$
FERMILAB SURUEY/ALIGNMENT

$J O E$ RECAP REPORT

Fage 4

JOB FILE: D-O DETECTOR INSIDE END CAP REFERENCE (cont' $d$ )

\begin{tabular}{|c|c|c|c|c|c|c|}
\hline PT NAME & $-x$ & 2 & $Y$ & DISTANCE & D AXES & DAT \\
\hline ORIENT & DOUBT & DOUET & DOUBT & CLOSURE & TEMP & TIM \\
\hline 000002 & 72.7593 & -34.5031 & $-82.4 \mathrm{~B} 17$ & 146.2812 & $--Z-Y$ & $10 / 21 / 87$ \\
\hline SOUTH & 0.0003 & 0.0209 & 0.0005 & 0.0009 & $70 \mathrm{~F}$ & $19: 24$ \\
\hline RE & RENCE VALUE & POINT NAME & FOR THE FO & LOWING POINT & WAS: & NEBALL \\
\hline $\begin{array}{l}000003 \\
\text { SOUTH }\end{array}$ & 80.8265 & $27.4 \equiv \equiv 6$ & $\begin{array}{r}-78.0045 \\
0.00005\end{array}$ & $\begin{array}{r}62.5419 \\
0.0917\end{array}$ & $\begin{array}{r}--z-Y \\
70 F\end{array}$ & $10 / 21 / 8$ \\
\hline
\end{tabular}

***** END OF POINTS LSED TO CREATE THE SOUTH ORIENTATION

SHOT ON SPIDER MIC IN CENTER BORE (SOUTH END 5 INCHES IN)

$\begin{array}{rrrrrrr}\text { S-TGT } & -0.5344 & -46.4739 & 0.2794 & 134.9574 & --Z-Y & 10 / 21 / 87 \\ \text { SOUTH } & 0.0006 & 0.0212 & 0.0007 & 0.0004 & 70 \mathrm{~F} & 20: 27\end{array}$

MAIN RING OUERPASS PIPE

$\begin{array}{lrrrrrr}\text { MRPIPE } & -12.8208 & -53.0842 & 81.7505 & 82.6571 & --Z-Y & 10 / 21 / 87 \\ \text { SOUTH } & 0.0007 & 0.0212 & 0.0012 & 0.0085 & 70 \mathrm{~F} & 20: 37\end{array}$

SHOOT TANGENT POINTS GF SOUTH ENO CAP (PUNCHES S1-54)

\begin{tabular}{|c|c|c|c|c|c|c|}
\hline $\begin{array}{l}51 \\
50 \cup T H\end{array}$ & $\begin{array}{r}-0.5212 \\
0.0006\end{array}$ & $\begin{array}{r}-58.4548 \\
0.0211\end{array}$ & $\begin{array}{r}48.6727 \\
0.0005\end{array}$ & $\begin{array}{r}35.6983 \\
0.0050\end{array}$ & $\begin{array}{r}--Z-Y \\
70 \mathrm{~F}\end{array}$ & $\begin{array}{r}10 / 21 / 87 \\
21: 11\end{array}$ \\
\hline $\begin{array}{l}\text { SZ } \\
\text { SOUTH }\end{array}$ & $\begin{array}{r}44.7037 \\
0.0003\end{array}$ & $\begin{array}{r}-58.3236 \\
0.0209\end{array}$ & $\begin{array}{l}1.4886 \\
0.2005\end{array}$ & $\begin{array}{r}65.3578 \\
0.0018\end{array}$ & $\begin{array}{r}--Z-Y \\
70 F\end{array}$ & $\begin{array}{r}10 / 21 / 87 \\
21: 12\end{array}$ \\
\hline $\begin{array}{l}\text { SE } \\
\text { SOUTH }\end{array}$ & $\begin{array}{r}-1.0589 \\
0.0005\end{array}$ & $\begin{array}{r}-58.1201 \\
0.8211\end{array}$ & $\begin{array}{r}-45.8534 \\
0.0004\end{array}$ & $\begin{array}{r}65.8448 \\
0.0040\end{array}$ & $\begin{array}{r}--Z-Y \\
70 \mathrm{~F}\end{array}$ & $\begin{array}{r}10 / 21 / 87 \\
21: 13\end{array}$ \\
\hline $\begin{array}{l}\text { \$4 } \\
\text { SOUTH }\end{array}$ & $\begin{array}{r}-47.3502 \\
0.0011\end{array}$ & $\begin{array}{r}-58.1558 \\
0.0215\end{array}$ & $\begin{array}{l}2.2738 \\
0.2008\end{array}$ & $\begin{array}{r}66.7765 \\
0.0005\end{array}$ & $\begin{array}{r}--Z-Y \\
70 \mathrm{~F}\end{array}$ & $\begin{array}{r}18 / 21 / 87 \\
21: 16\end{array}$ \\
\hline
\end{tabular}

PUTTING A PUNCH MARK ON THE INSIOE OF THE CENTER EORE (SOUTH END TOP)

$\begin{array}{lrrrrrr}\text { S-BORE } & 0.0025 & -47.7394 & 32.5950 & 57.1910 & --Z-Y & 10 / 21 / 87 \\ \text { SOUTH } & 0.0005 & 0.22: 2 & 0.0009 & 0.0001 & 70 \mathrm{~F} & 21: 37\end{array}$

CLOSE ON SE-TGT

$\begin{array}{lrrrrrr}\text { CLOSE } & 72.7588 & -34.5110 & -82.4803 & 136.7874 & --2-Y & 10 / 21 / 87 \\ \text { SOUTH } & 0.0002 & 0.0209 & 0.0005 & 0.0002 & 70 \mathrm{~F} & 21: 40\end{array}$


$10 / 22 / 87$

$21: 38$
FERMILAB SURUEY/ALIENMENT

I OB RECAP REFORT

JOB FILE: D-O DETECTOR INSIDE END CAP REFERENCE (cont'd)

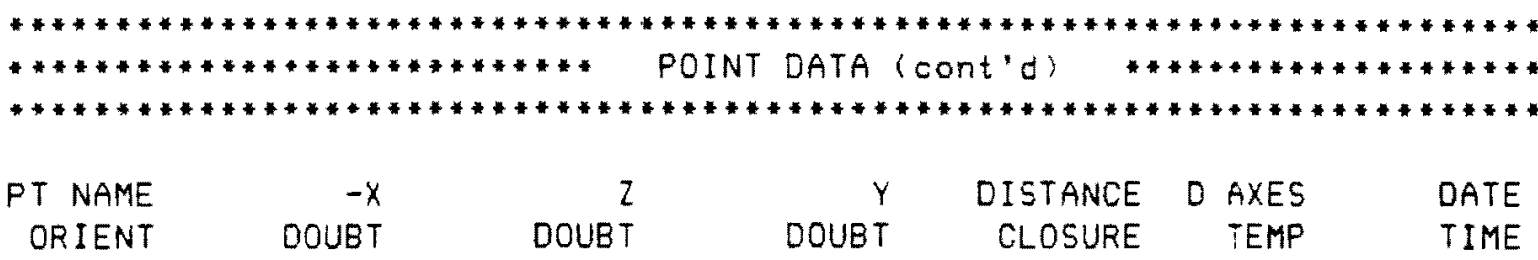

* thE NEXT 3 POINTS WERE USED tO CREATE THE NORTH ORIENTATION **

REFERENCE UALUE FOINT NAME FOR THE FOLLOWING POINT WAS: SEBALL
000004
$80.575 e^{2}$
$-28.1836$
$-77.9174$
0.000
$--z-y$
$10 / 22 / 87$
NORTH
$0.000 \mathrm{~s}$
0.0005
$0.0023 \quad 70$
$18: 52$

REFERENCE UALUE POINT NAME FOR THE FOLLOWING POINT h4S: NETGT
000005
$72.795 !$
34.2180
$-82.5211$
115.2377
$--z-y$
$10 / 22 / 87$
NORTH
0.0003
0.0008
0.0004
$0.000370 \mathrm{~F}$
$18: 58$

REFERENCE VALUE POINT NAME FOR THE FOLLOWING POINT WAS: NWTGT
000006
$-73.1999$
34.5242
$-82.1275$
145.9958
$--z-Y$
$10 / 22 / 87$
NORTH
0.0015
0.0020
0.0006
0.0006
$70 \mathrm{~F}$
$19: 01$

***** END OF POINTS USED TO CREATE THE NORTH ORIENIATION

SHOT ON SPIDER MIC IN CENTER BORE (NORTH END 5 INCHES IN)
$N-T G T$
$-0.0790$
46.4629
0.2231
110.7737
$--Z-Y$
$10 / 22 / 87$
NORTH
0.0009
0.0010
0.0008
$0.0019 \quad 70 \mathrm{~F}$
$19: 09$

MAIN RING OUERPASS PIPE
PIPEMR
NORTH
-12.9262
0.0012
53.6508
0.0010
81.4749
0.0015
$\begin{array}{rr}82.5755 & --Z-Y \\ 0.0059 & -0 \mathrm{~F}\end{array}$
$10 / 22 / 87$
$19: 17$

NOTE: OUERFASS PIPE WELOS WERE CUT PRIOR TO THIS SHET

SHOOT TANGENT POINTS ON NORTH END CAP (PUNCHES N1-NE)

$\begin{array}{lrrrrrr}\text { N1 } & 1.0603 & 58.6049 & 49.1727 & 35.5457 & --Z-Y & 10 / 22 / 87 \\ \text { NORTH } & 0.0009 & 0.0009 & 0.0011 & 0.0006 & 70 \mathrm{~F} & 20: 57 \\ \text { N2 } & 48.2352 & 58.4700 & 0.4149 & 57.8440 & --Z-Y & 10 / 22 / 87 \\ \text { NORTH } & 0.0204 & 0.0007 & 0.0005 & 0.0012 & 70 \mathrm{~F} & 20: 59 \\ \text { N3 } & -0.45 E 5 & 58.8684 & -46.8219 & 57.8687 & --Z-Y & 10 / 22 / 87 \\ \text { NORTH } & 0.0029 & 0.0009 & 0.0004 & 0.0020 & 70 \mathrm{~F} & 21: 01 \\ \text { N4 } & -46.2673 & 58.7351 & 0.1819 & 55.0082 & --Z-Y & 10 / 22 / 87 \\ \text { NORTH } & 0.005 & 0.0012 & 0.0009 & 0.0074 & 70 \mathrm{~F} & 21: 03\end{array}$


$A^{-1}$

$10 / 22 / \varepsilon ?$
$21: 38$

FERMILAB SURUEY/ALIGNMENT
$J O B$ RECAP REPORT

JOB FILE: D-D DETECTOF INSIDE END CAP REFERENCE (cont'd)

PT NAME ORIENT

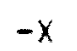

* CLOSE

72.7974

NORTH
34.2190

0.0008
$-82.5225$

0.0004
147.0288

0.0019
$--Z-Y$

$70 \mathrm{~F}$
$10 / 22 / 87$

$21: 06$ 


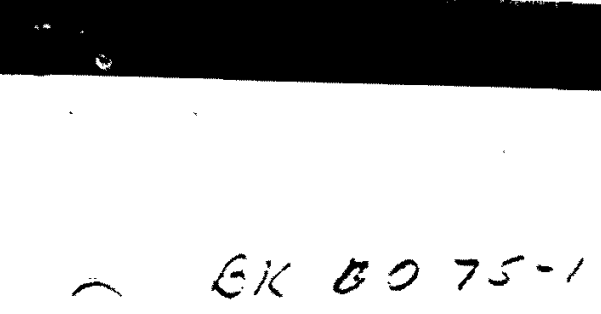

$4 \cdot 15$

10

$10: 10 / 87$

$2: 20$

FERMILAE SURUEYIALI INTENT

Page 10

JOE FILE: DOE DETECTOR ENC CAP REFERENCE

OPERATORS: JAMES DAMLBERE

LOCATION: LAB A

MEASUREMEN UNITS: InChES

COMMENTS:
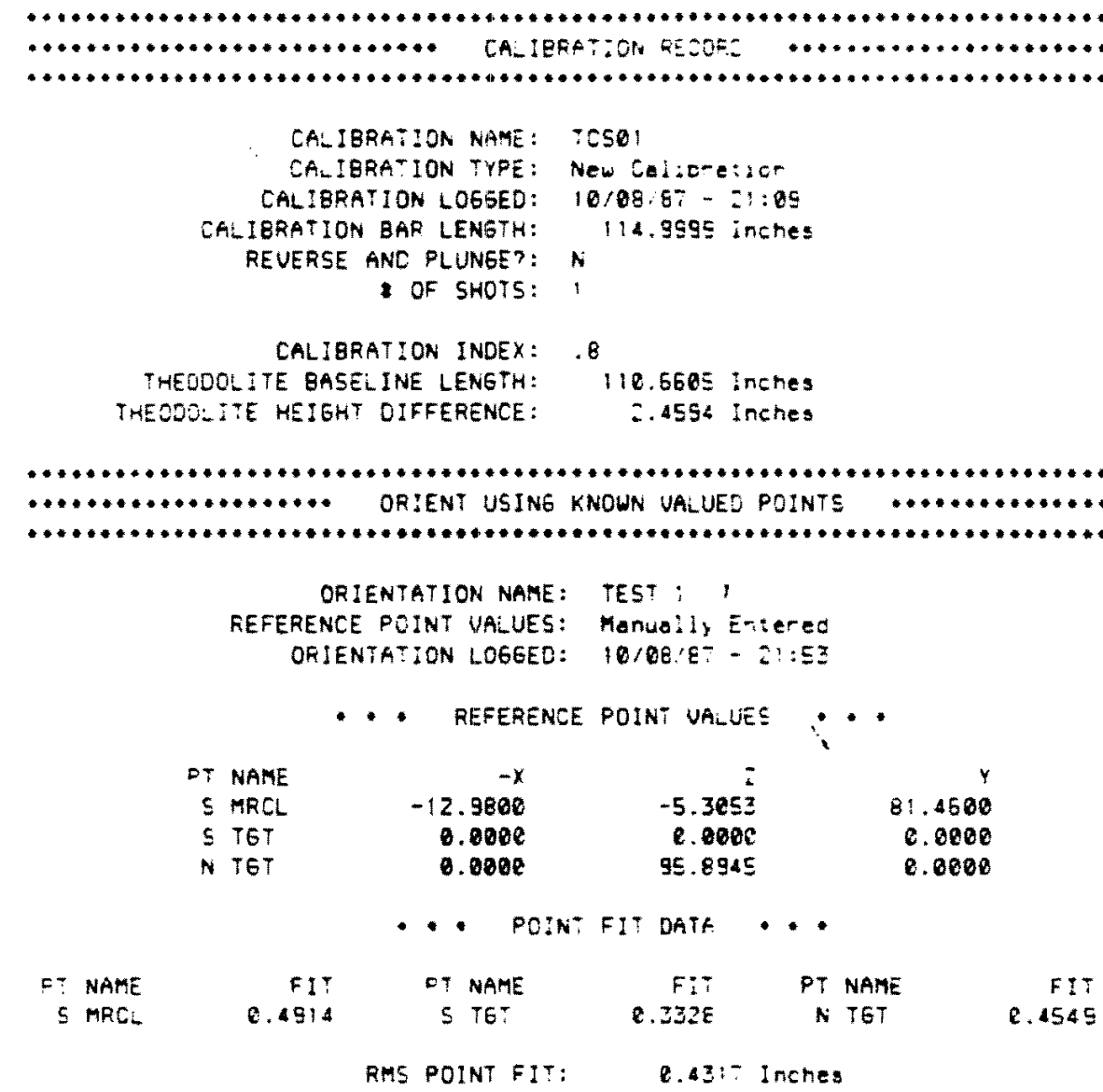

RMS POINT FIT:

Q.43:- Inches 


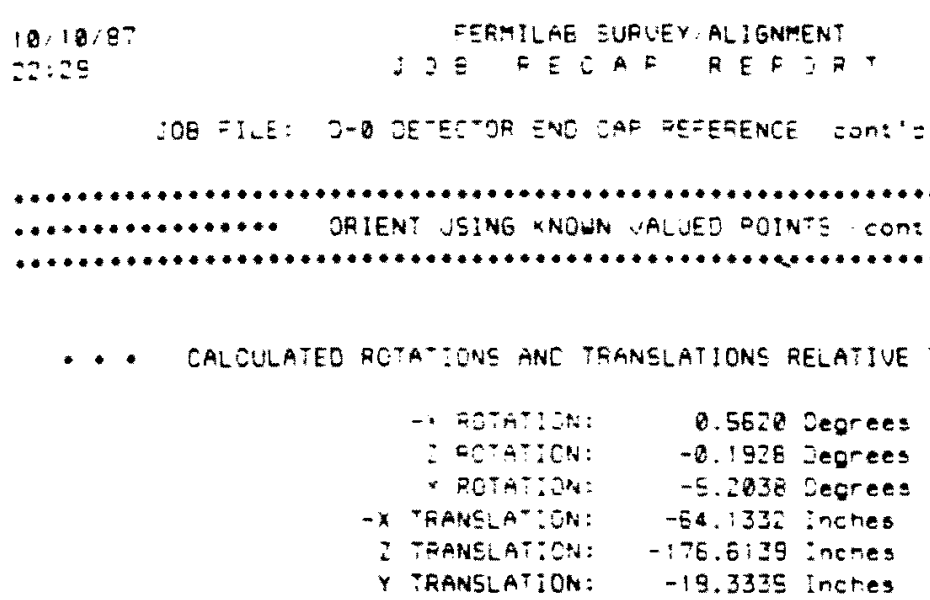


BK DO, $75-1$

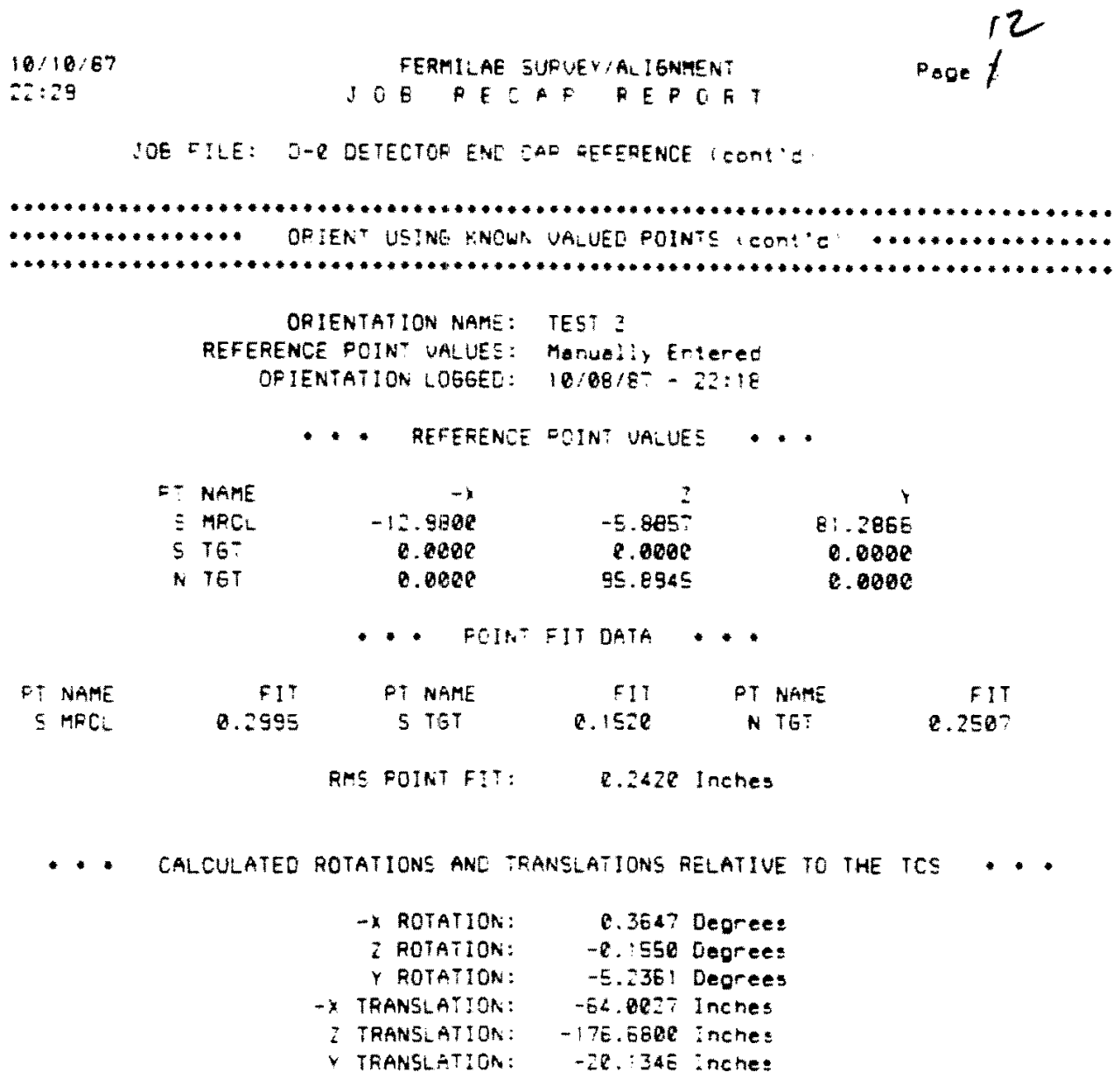

$4-17$ 


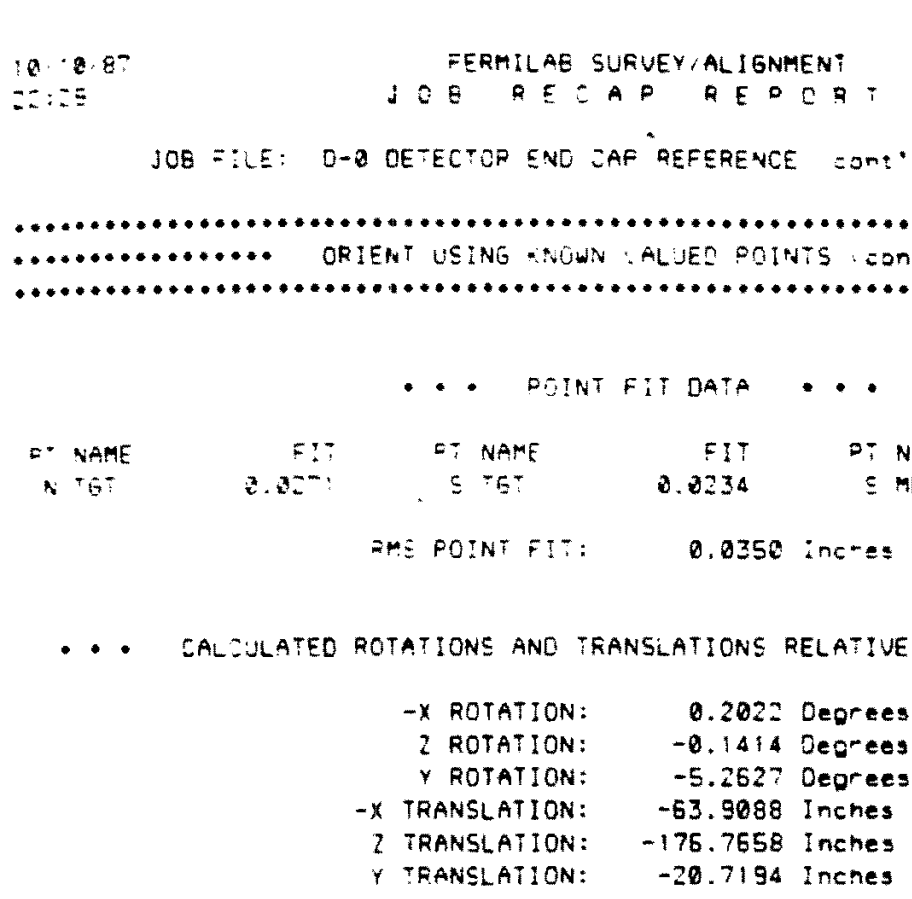

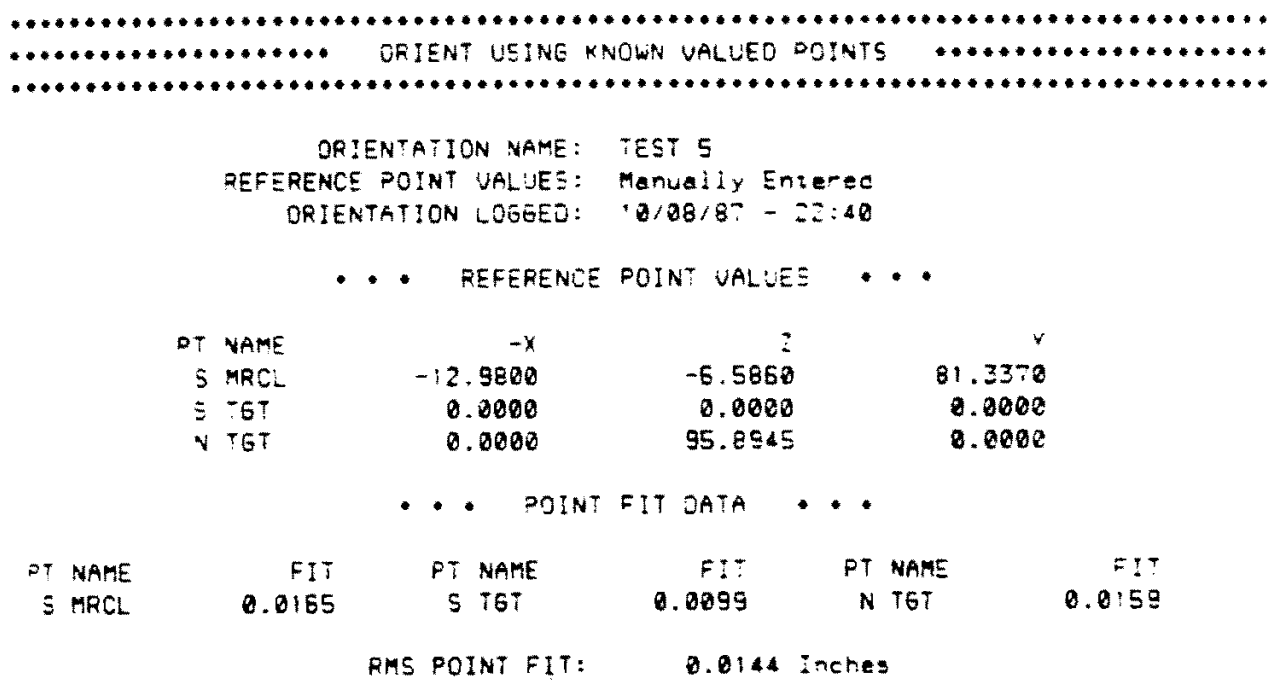




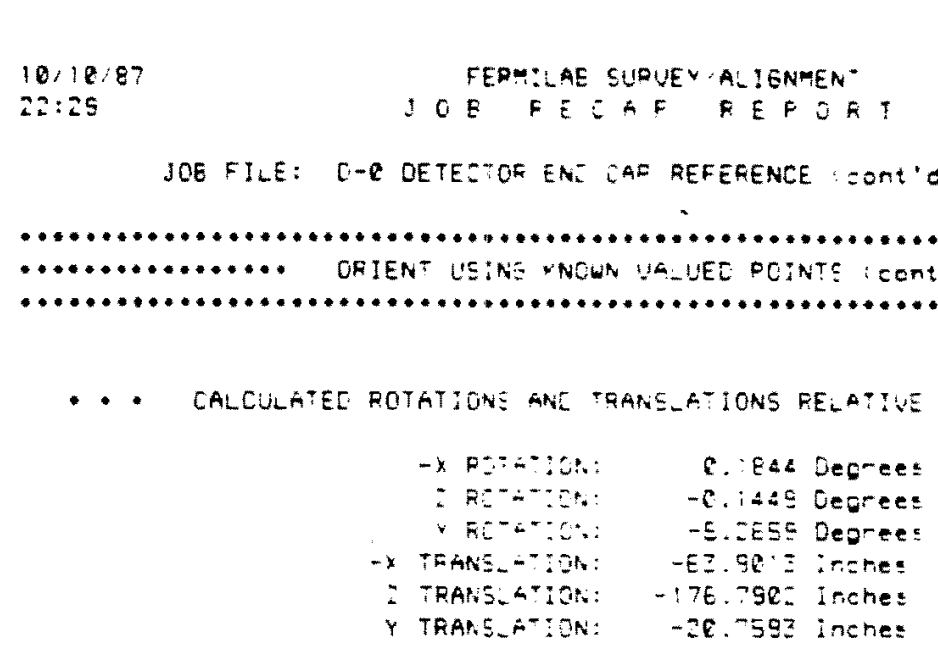

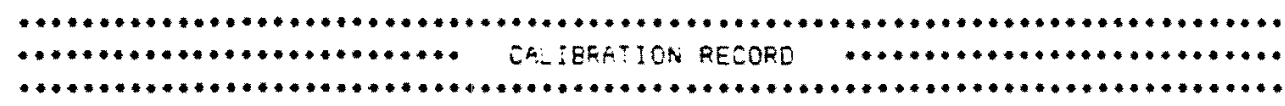

CALIBRATION NAME: TCSOZ

CALIBRATIOK TYPE: NE COLIDTA:ion

CALIBRATION LO6EEL: 10.09,87-17:38

CALIBPATION BAF LENETH: 112.9999 inches

REUERSE ANO PLUNEE;:

- OF SHOTS:

CALIBRATION INDEX:

THEODOLITE BASELINE LENETH:

THEOOOLITE MEIGHT OIFFERENSE:

107.0774 inches

14

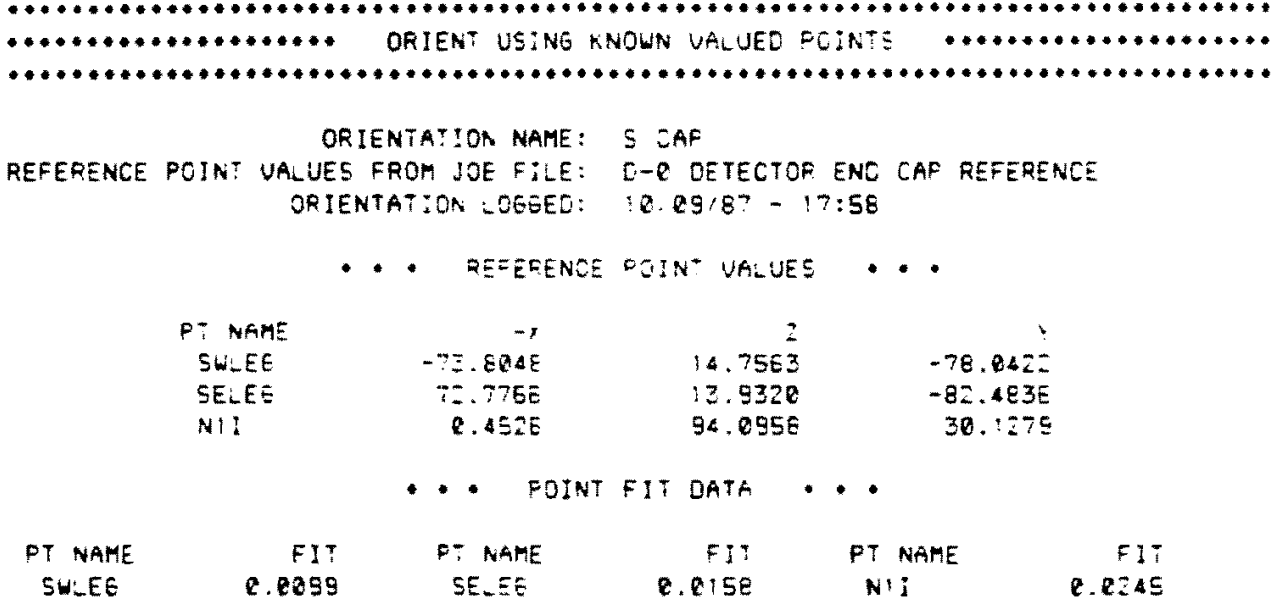



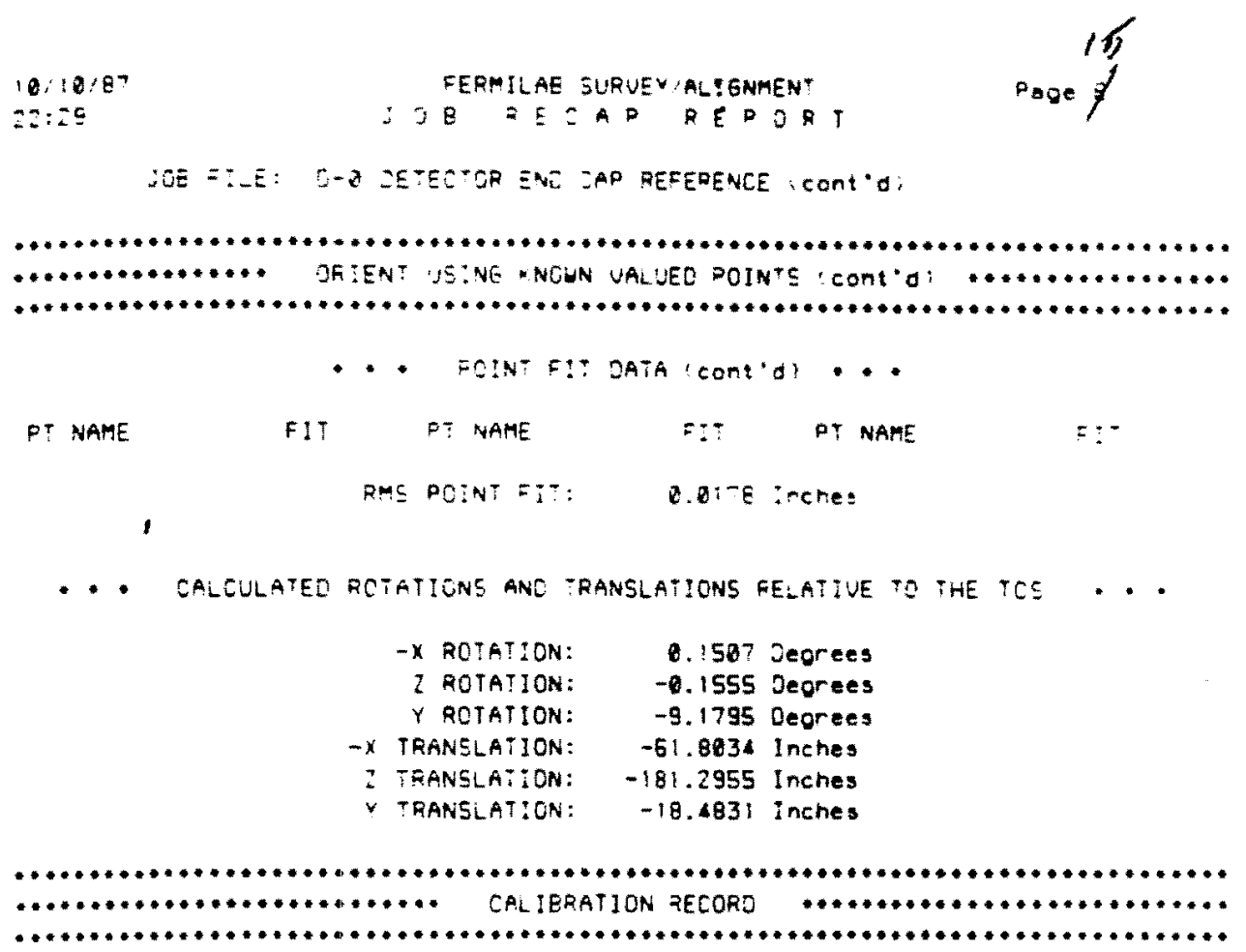

CAL:BFATION NAME: TCSOS

CALIIERATION TYPE: New Calibration CAL!ERAT:ON LO66ED: 10/09/8? - 22:40 ZALIBRTTION BAF LENGTH: 1:4.5959 inches REVEESE AND OLUNEE?: N

- OF SHET:

GALIBFGTION INCEX: 2.2 THEOCOLITE BASELINE LENE:H: THEOOOLITE HEIGHT DIFTERENCE:

144.2450 inches

4.6026 inches

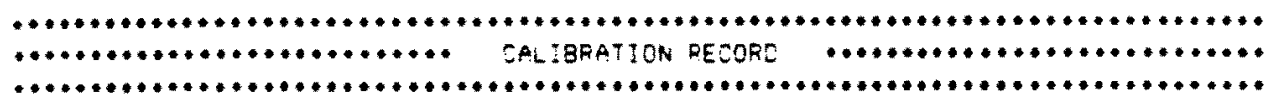

CALIERATION NAME: TCSOL

CALIERATION TYPE: New CAlibration

CALIBRATION LOE6ED: $10 / 09 / 87-22: 46$

CALIBRATION BAR LENGTH: 114.9995 Inches

REVERSE AND PLUNGE?:

I OF SHOTS:

CAL IBFAT:ION INCEX:

THEODOLITE BASEL:NE LENGTH:

THEOOOLITE ME: EHT OIFFERENCE:

1.9

14.2448 Inehos

4.6045 Inches 
3

$* 4$

Ex $0075-1$

$A \cdot 21$

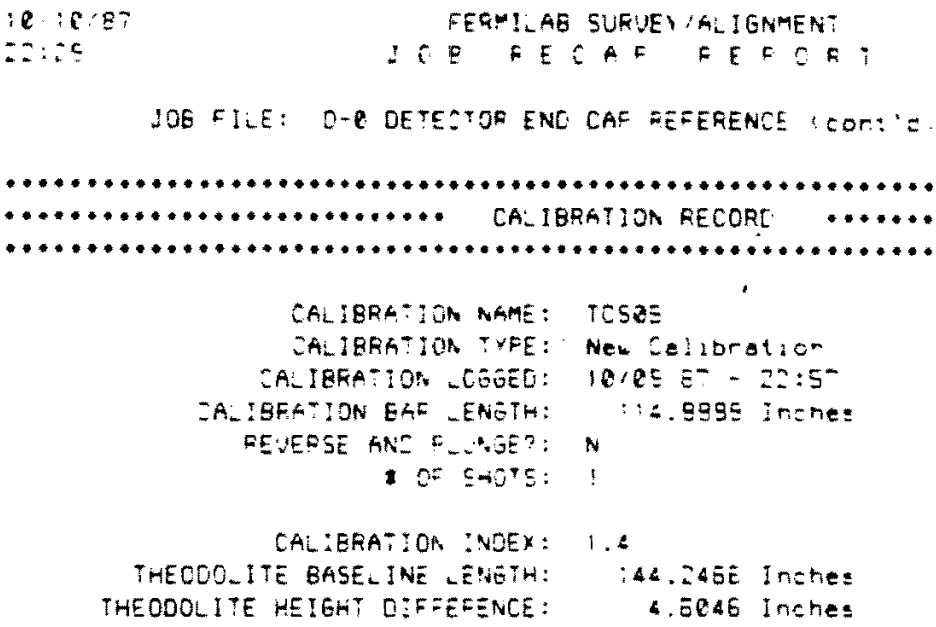




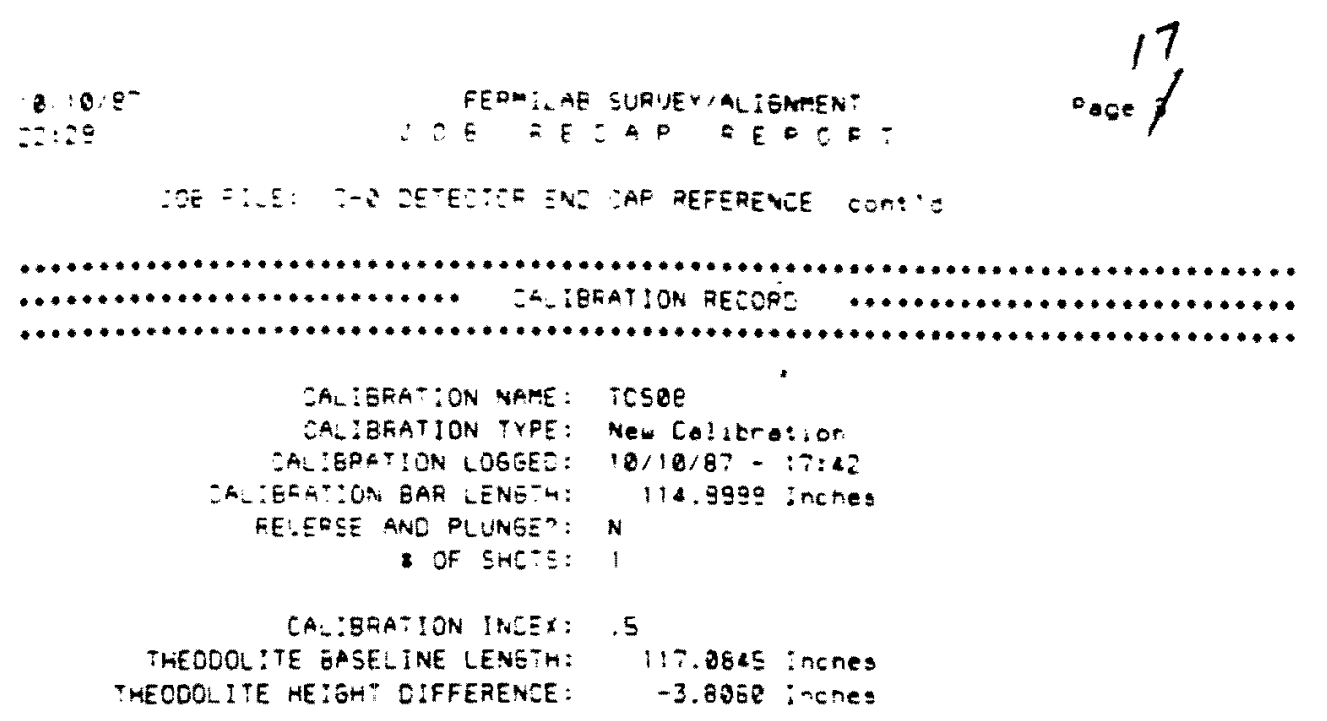

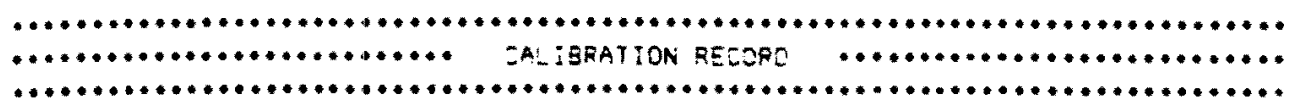

CAL:BRATION NAME:

GAL:EPATION TYOE: EAL:EFATIOH LOEEED: ZA_ZEE-TON BAR LENEZM: FE:EZCE ANE PULPE: : o of $5-\div: 5: 1$ ZA:ZEFTION : VEER:

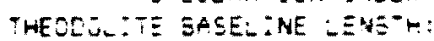
THEORO: :
TC509

New Caliteaticr

(6):10.87-ie:05

114. 9EE inches

1

$1: .08 \equiv \equiv$ :nches

Z. $3 \mathbb{Q}^{- \pm}$inches

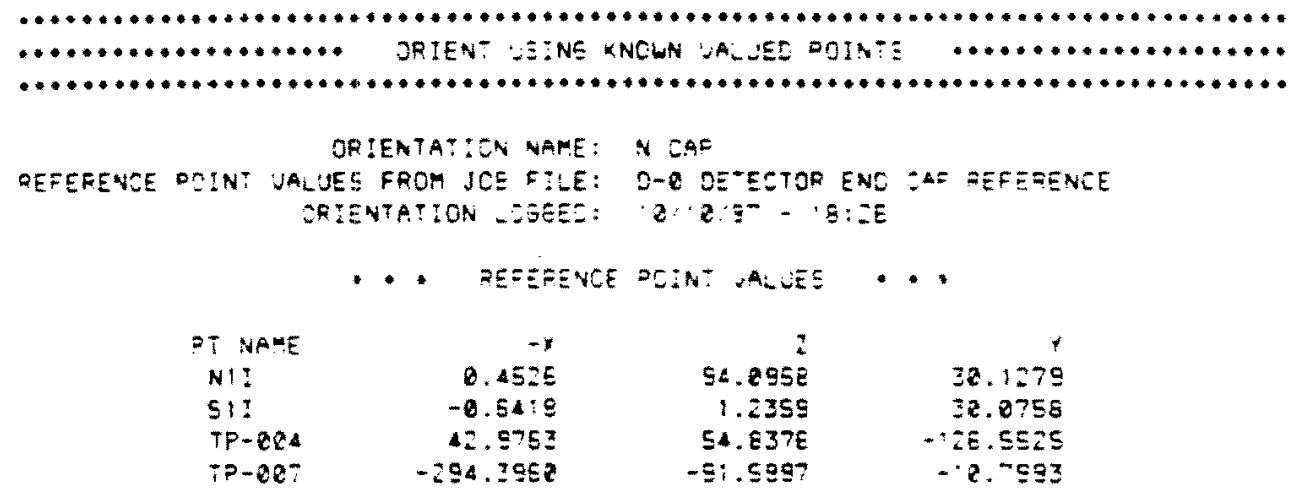




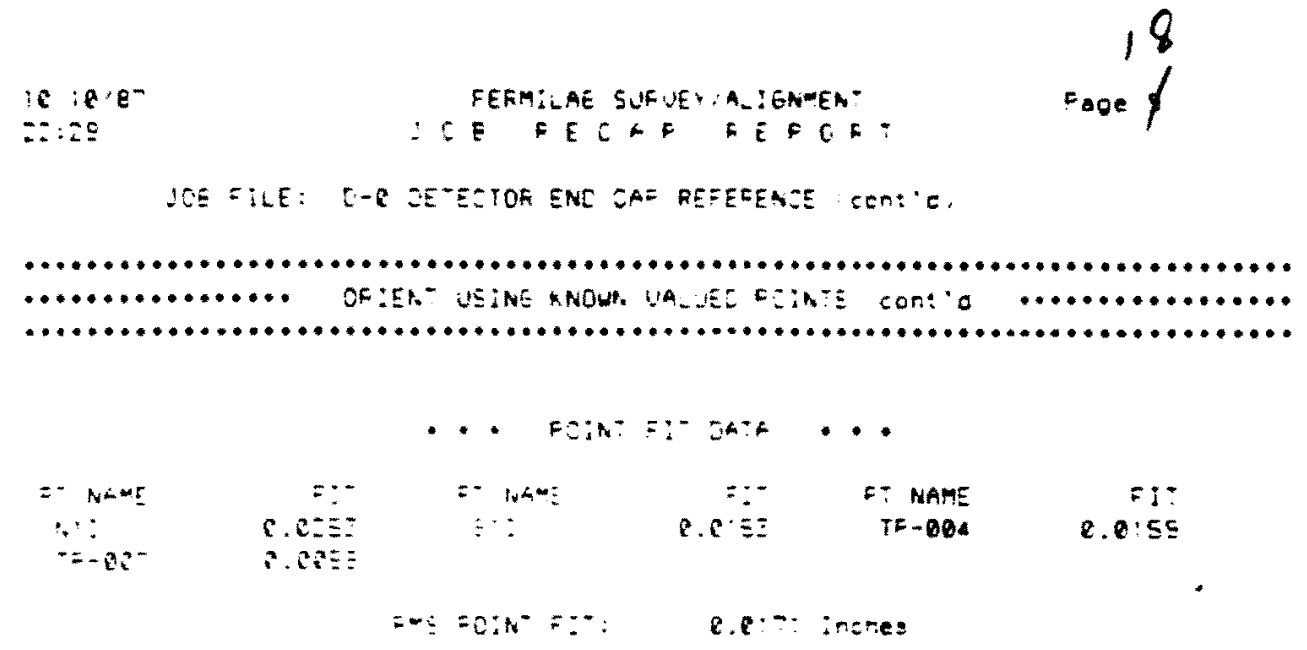

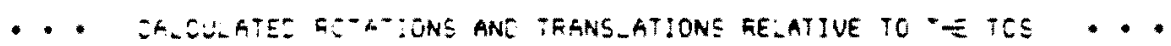

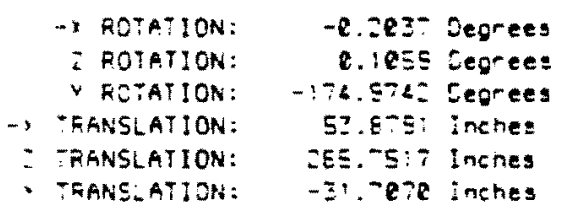

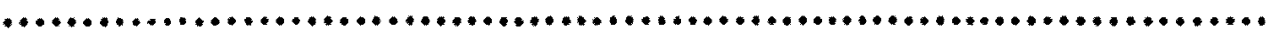

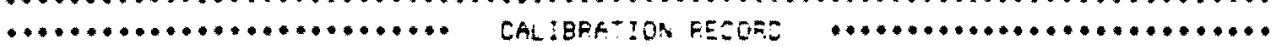

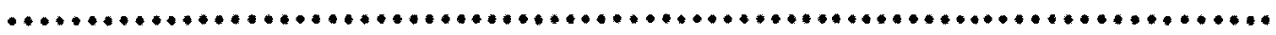

CAMBBFE:ON NAME: TCSIE

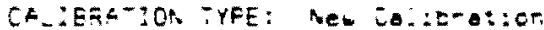

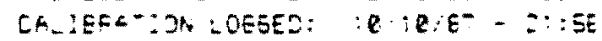

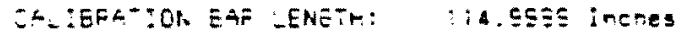

PEUEFSE AME FLUNEE":

1) OF SHoTs:

CA_ IBEF::ON INDEX: THESZOUTE BASE_:NE LENETH: THEOSO_:TE HEIGH" CIEFERENEE:

$\pm$

104.219: Inches

-1.49 in inches

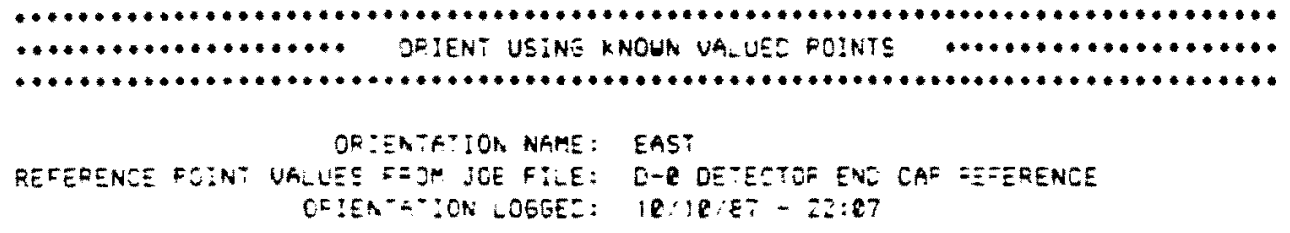




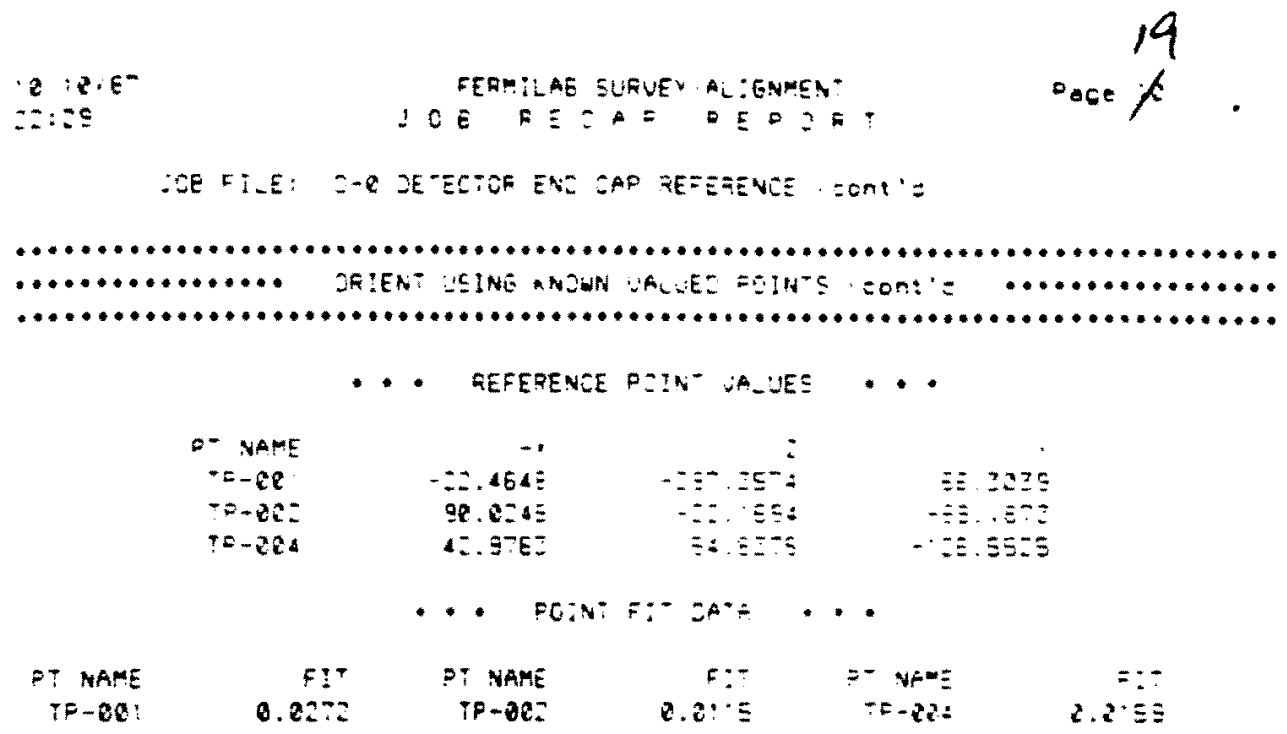

$$
\text { RMS DOINT FIT: } \quad \boldsymbol{Z} \text {. } 2 \text { : } 9 \mathrm{~A} \text { inehe: }
$$

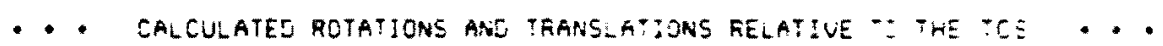

$$
\begin{aligned}
& \text { - FOTA:ECN: } \\
& \text { I ROTAT:OH: } \\
& \text { Y ROTATION: } \\
& \text { - TRANSLATION: } \\
& \text { - TANELATICN: } \\
& \text { Y TRANSLAT:CN: } \\
& \text { - zecs cegrees } \\
& \text {-3. } \$ E 4 \text { Degree: } \\
& -12 a .5905 \text { Deorees } \\
& 251.41: 8 \text { incties } \\
& \text { : ze el? incres } \\
& \text { - 4. :5:3 inones }
\end{aligned}
$$

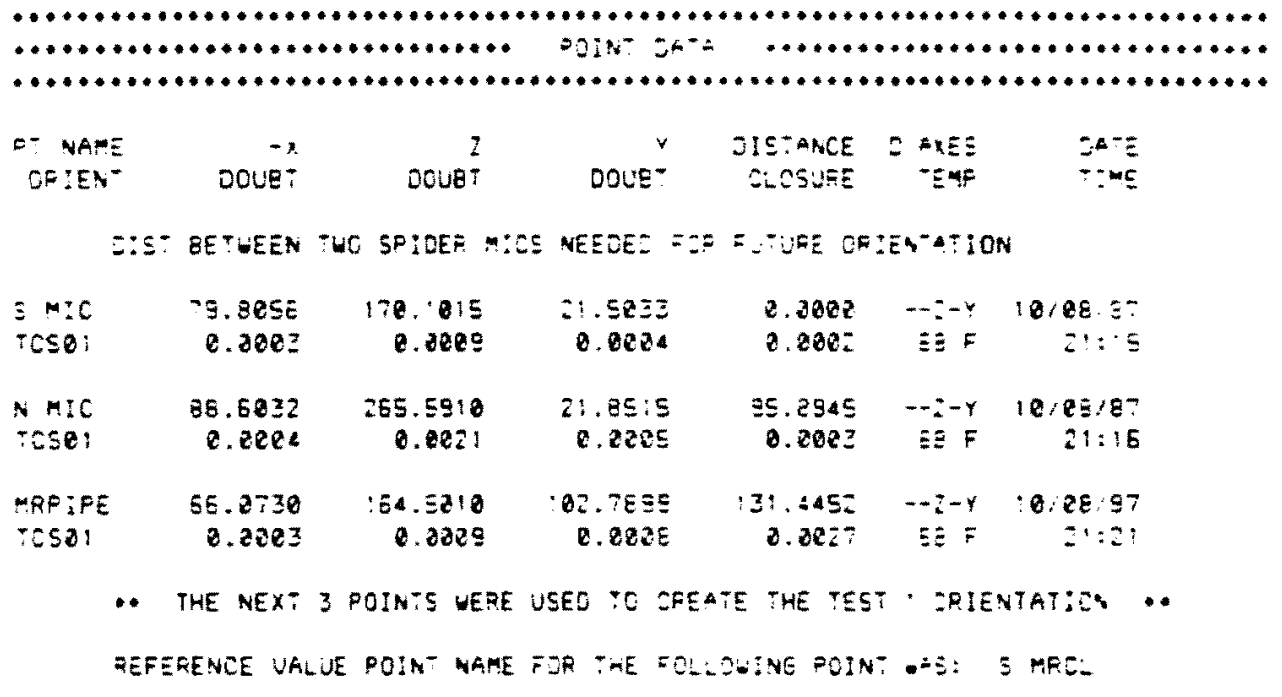




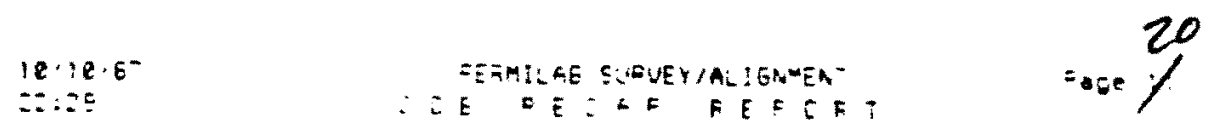

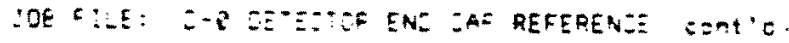

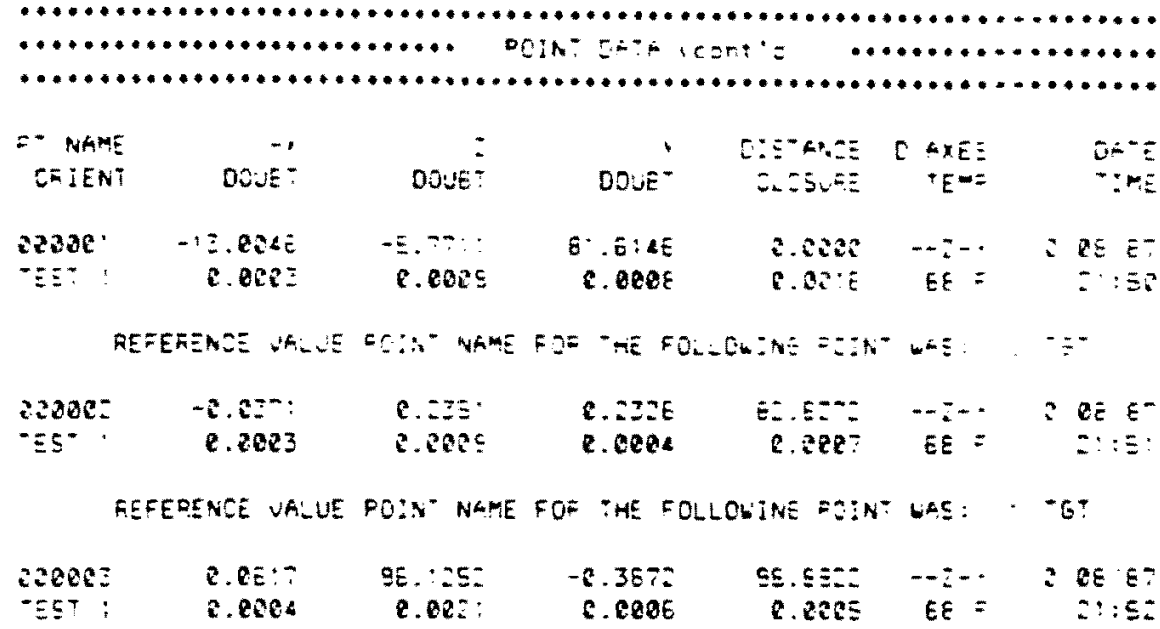

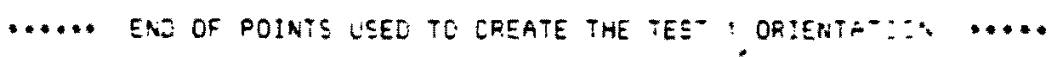

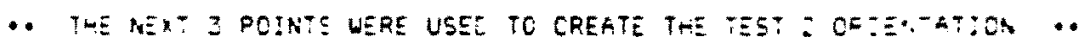

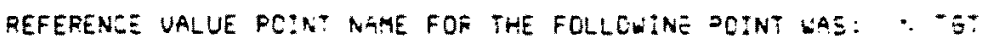

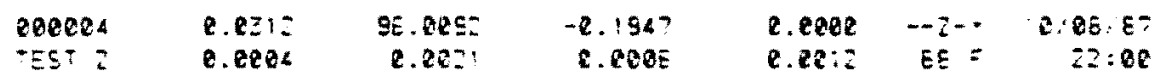

PEFERENEE UALUE POEN KAME FOE THE FOLLOHNE SOINT WLS: :-"E-

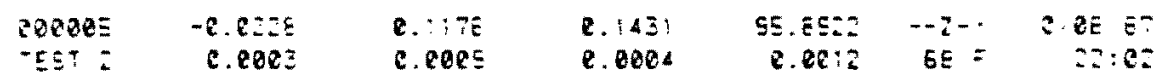

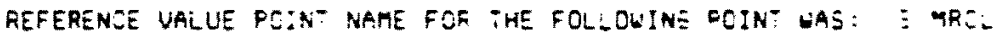

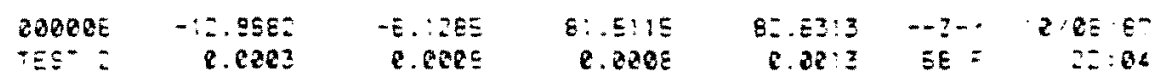

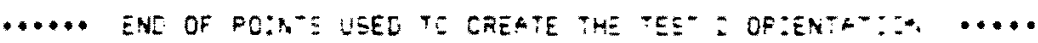

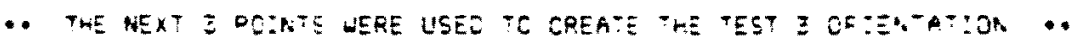

FEFERENEE VALUE POIR: NAME FOF THE FOLLOWINE POINT VAS: I MAC:

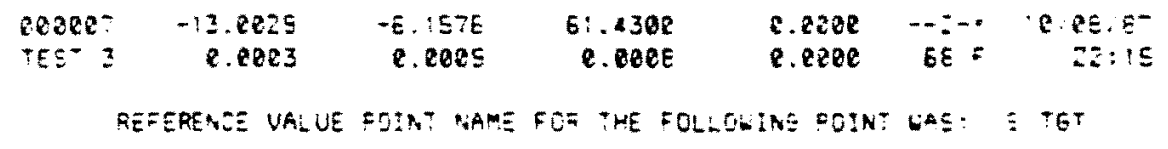

\begin{tabular}{|c|c|c|c|}
\hline $\begin{array}{l}\text { rocace } \\
755 \%\end{array}$ & $\begin{array}{r}- \text { e.e:11 } \\
\text { C.ezez }\end{array}$ & $\begin{array}{l}\text { c. }: 345 \\
\text { e.2ens }\end{array}$ & $\begin{array}{l}\text { R. } 2 E 95 \\
\text { 2. aBe4 }\end{array}$ \\
\hline
\end{tabular}




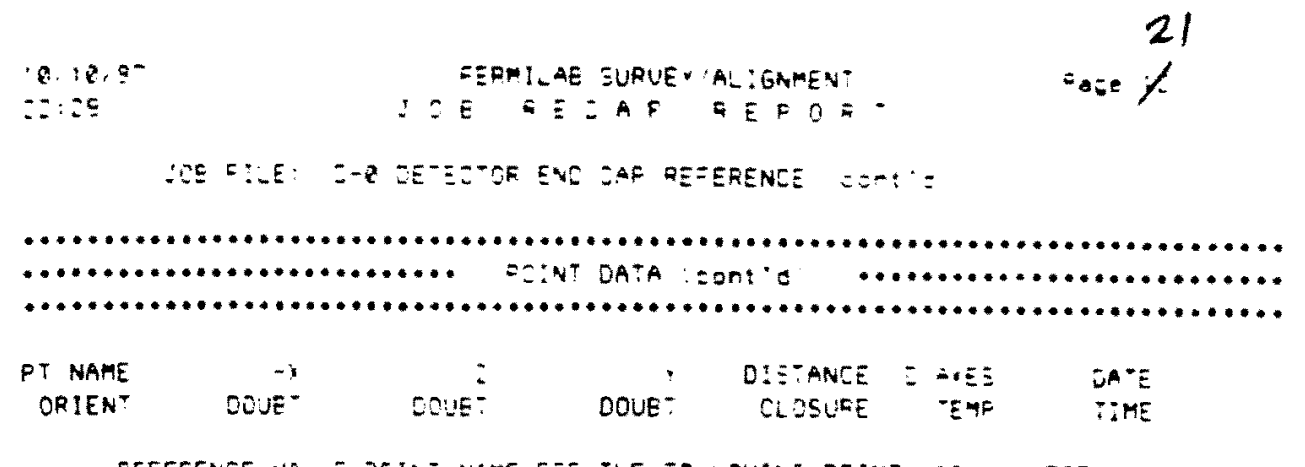

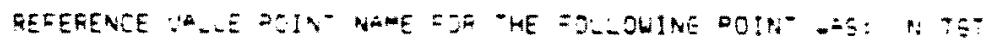

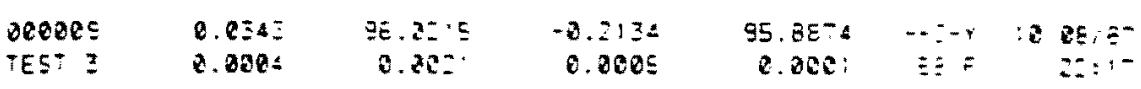

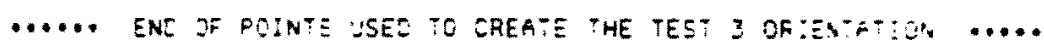

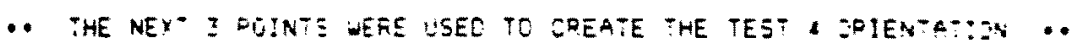

REFERENCE VALUE POIN" NAME TOR THE FOLLOUING POINT ALE: N TE:
000010
TES? 4
2. 0038
SE. $E R:$
$-0.0242$
0.008
$--z-1$
: $: 2: 2: 27$

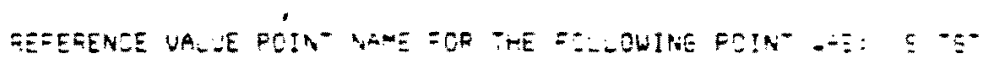

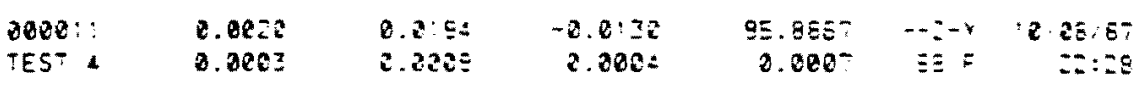

REFEPENCE VALE POIN VAME EEF THE FZLLWINE OOIN"

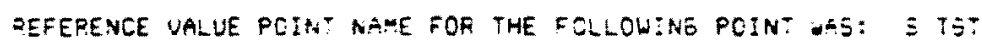

\begin{tabular}{|c|c|c|c|c|c|}
\hline $\begin{array}{l}2200: 4 \\
\text { TFST }\end{array}$ & $\begin{array}{l}2.0211 \\
0.0002\end{array}$ & $\begin{array}{r}-2.20 E E \\
2.2020\end{array}$ & $\begin{array}{r}-2.0072 \\
0.200=\end{array}$ & $\begin{array}{r}82.6287 \\
0.0022\end{array}$ & $\begin{array}{r}10.85 \\
2: 39\end{array}$ \\
\hline
\end{tabular}

REFERENCE HALUE PGIA: WAYE FOR THE FOLLOWING POINT JAS: N TE"

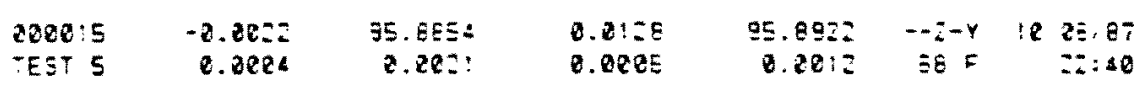

...... ENO OF POINTS JSED TO CREATE THE TEST S OE:ENTATIJN ..... 


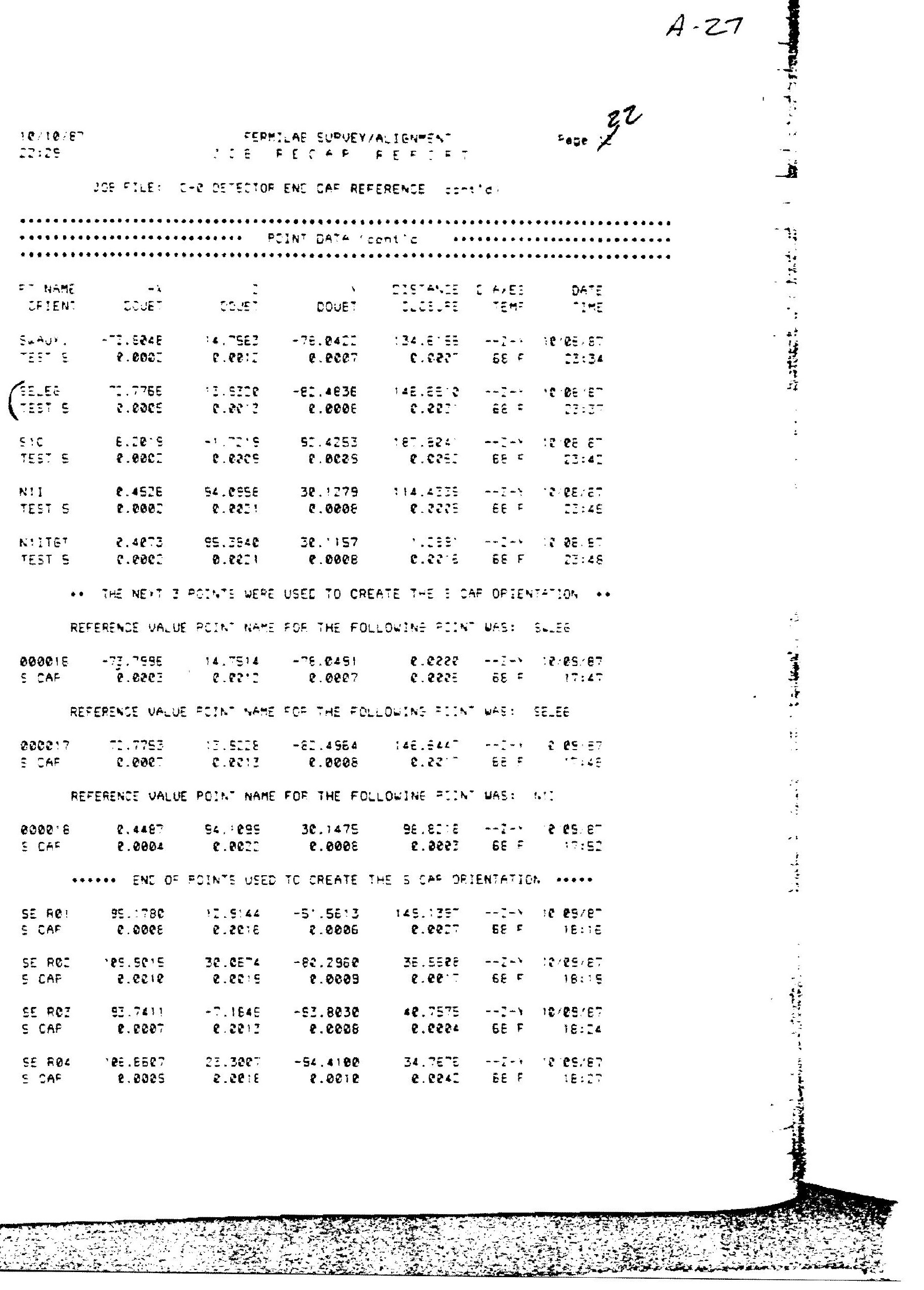




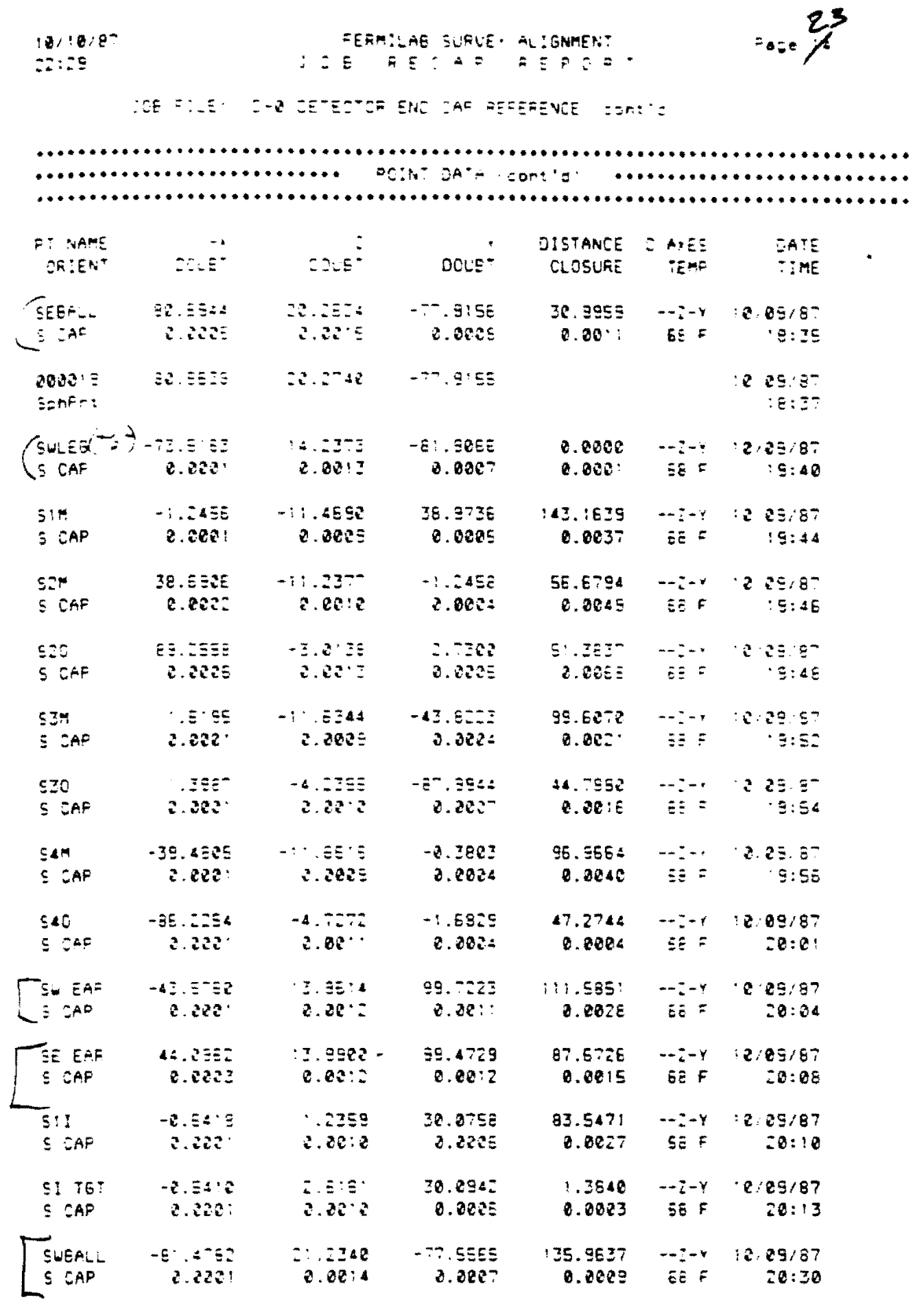




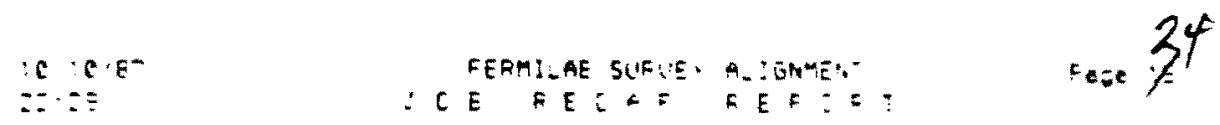

ZDE FILE: C-P DETESTOF ENE CAL FETERENEE COM:' $C$

\begin{tabular}{|c|c|c|c|c|c|c|}
\hline $\begin{array}{l}=- \text { NAME } \\
==\text { EEN }\end{array}$ & $\begin{array}{c}-3 \\
\text { Sove* }\end{array}$ & S5U: & atis: & $\begin{array}{r}\text { SETACE } \\
\text { ERE }\end{array}$ & $\begin{array}{r}P 4 \times E \equiv \\
+5 \times=\end{array}$ & $\because=$ \\
\hline $\begin{array}{l}-c-b e: \\
\vdots: F=\end{array}$ & 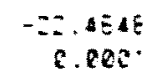 & $\begin{array}{r}-5 . .3574 \\
\text { c.cores }\end{array}$ & $\begin{array}{r}E .3 e=5 \\
\text { e.ece }\end{array}$ & $\begin{array}{r}256.3= \\
\text { c.02: }\end{array}$ & $B E=$ & 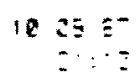 \\
\hline $\begin{array}{l}=-\mathrm{ez:} \\
=\therefore:\end{array}$ & $\begin{array}{r}\text { se.cost } \\
\text { c.cose }\end{array}$ & $\begin{array}{r}-: \text { : 1694 } \\
\text { e.ce:: }\end{array}$ & $\begin{array}{r}-85.18= \\
\text { 6.000 }\end{array}$ & $\begin{array}{r}2 x E \\
\text { C.232 }\end{array}$ & $\begin{array}{l}--\Xi- \\
E \equiv\end{array}$ & $\begin{array}{r}\text { 10. } \\
\square= \\
=:\end{array}$ \\
\hline $\begin{array}{l}=-28 z \\
=2 \alpha= \\
z=2\end{array}$ & $\begin{array}{l}3 E \\
\text { C.R2E: }\end{array}$ & $\begin{array}{r}-4 \varepsilon .7:: \\
\text { C.c2as }\end{array}$ & $\begin{array}{r}-Z-. \overline{D E}= \\
\text { C.OR: }\end{array}$ & $\begin{array}{r}z 9 . \equiv Z \equiv: \\
\text { c.22:2 }\end{array}$ & $\begin{array}{c}--Z-= \\
\text { EE }=\end{array}$ & $\begin{array}{r}\text { : } 25 \equiv \\
\because:\end{array}$ \\
\hline $\begin{array}{l}=-204 \\
\equiv=4 .\end{array}$ & $\begin{array}{r}\text { 4. E7EZ } \\
\text { c.2eza }\end{array}$ & $\begin{array}{r}51 . E=7 E \\
\text { R. REIE }\end{array}$ & $\begin{array}{r}-7 E .530 \equiv \\
\text { R.02: }\end{array}$ & $\begin{array}{r}245.44=2 \\
\quad 8.20=3\end{array}$ & $\begin{array}{l}--Z= \\
\text { EE }=\end{array}$ & 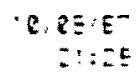 \\
\hline $\begin{array}{l}=-205 \\
\equiv=A F\end{array}$ & $\begin{array}{r}25 \% .275 z \\
\text { e.000 }\end{array}$ & $\begin{array}{r}=5 .-118 \\
8.8: 16\end{array}$ & $\begin{array}{r}-E . E S Z Z \\
\text { E.ReI: }\end{array}$ & $\begin{array}{r}402.84 E Z \\
e .02: 1\end{array}$ & $\begin{array}{l}--Z-V \\
6 E=\end{array}$ & 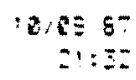 \\
\hline $\begin{array}{l}P=-2 B E \\
=C A F \\
\equiv C A\end{array}$ & $\begin{array}{r}-2 E: . E Z 7 E \\
\text { C.BE!E }\end{array}$ & $\begin{array}{r}\text { EE. E:8Z } \\
\text { C.e2इZ }\end{array}$ & $\begin{array}{r}-5.885: \\
\text { 2. } 2020\end{array}$ & $\begin{array}{c}626.2409 \\
\text { e.e2:1 }\end{array}$ & $\begin{array}{c}--z-Y \\
\text { EE F }\end{array}$ & $\begin{array}{r}10: 25=3 \\
=: 34\end{array}$ \\
\hline $\begin{array}{l}T E-2 C 7 \\
S \text { CAF }\end{array}$ & $\begin{array}{r}-294.3958 \\
\text { e.c025 }\end{array}$ & $\begin{array}{r}-51.5957 \\
\text { e.cezs }\end{array}$ & $\begin{array}{r}-12.7992 \\
\text { c.000E }\end{array}$ & $\begin{array}{r}2 E R . \equiv: 25 \\
0.22: 7\end{array}$ & $\begin{array}{c}--z-y \\
6 E=\end{array}$ & $\begin{array}{r}10 \\
-2 \\
-:=\end{array}$ \\
\hline $\begin{array}{l}=-8 e \varepsilon \\
\equiv C A=\end{array}$ & $\begin{array}{r}-8 E .-5 \geqslant 5 \\
\text { e. } 000:\end{array}$ & $\begin{array}{r}-15.3: 19 \\
\text { e.cele }\end{array}$ & $\begin{array}{r}-8 E .712 z \\
0.00 e^{-}\end{array}$ & $\begin{array}{r}229.45 E \\
\text { C.220E }\end{array}$ & $\begin{array}{r}--Z=v \\
E E=\end{array}$ & $\begin{array}{r}10<5: 5 \\
2::\end{array}$ \\
\hline
\end{tabular}

CLCEEOUT ON TAREE: SEA:X.

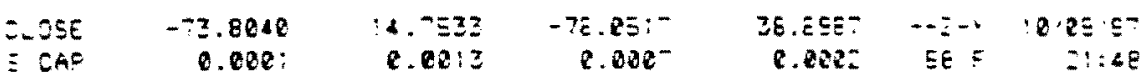

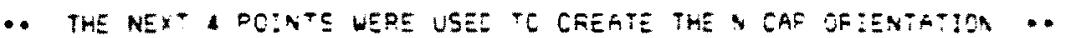

RE =ERENEE UALUE PE:N* NAME FOF THE FOLLOH:NE $=E \mathrm{IN}^{*}$ UAE: $\mathrm{N}^{*} \mathrm{i}$

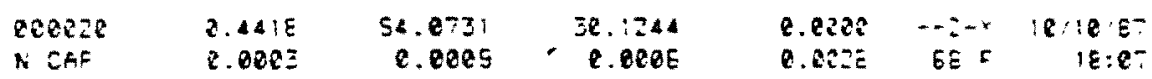

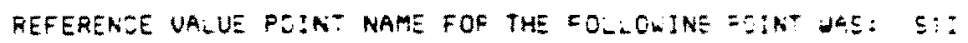

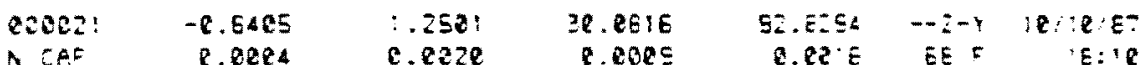

REFERENCE VALUE PE:N: NAME FOF THE FGLLOWNE OEIN: WFE: TE-2OL
eres::
$4: .9084$
$54.8447-125.5595$

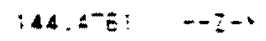
1e:1: : : ?

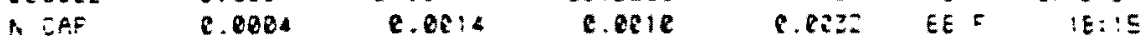


$10 \cdot 20$
$2:-29$

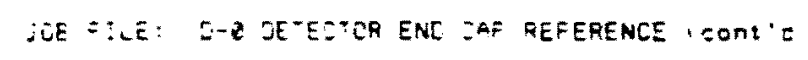

FEAMILAG SLRUEY/MLIENMEN:

$Z E R E: L F$ REDST $\ldots$

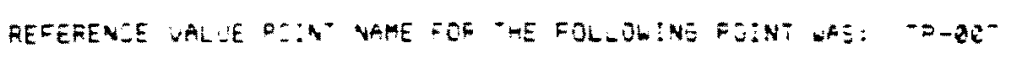

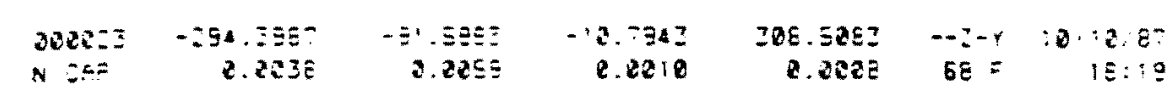

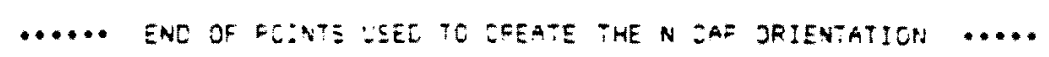

\begin{tabular}{|c|c|c|c|c|c|c|}
\hline $\begin{array}{l}\text { NWEA L } \\
\text { N BAF }\end{array}$ & $\begin{array}{r}-8.1140 \\
0.2004\end{array}$ & $\begin{array}{r}76.6 \div 12 \\
e .20: 5\end{array}$ & $\begin{array}{r}-77.7356 \\
\text { e.2206 }\end{array}$ & $\begin{array}{r}279.7555 \\
0.08 \cdot 5\end{array}$ & $\begin{array}{c}--2-y \\
58 F\end{array}$ & $\begin{array}{r}10 / 10: 87 \\
18: 34\end{array}$ \\
\hline $\begin{array}{l}\operatorname{Min}: S T \\
\therefore:==\end{array}$ & $\begin{array}{r}-2.22=1 \\
\text { 2.2004 }\end{array}$ & $\begin{array}{r}82.996 E \\
\text { Z.62: }\end{array}$ & $\begin{array}{r}-8: .2: 75 \\
\text { e. } .0306\end{array}$ & $\begin{array}{r}11.04 e^{-} \\
0.2 e^{2}\end{array}$ & $\begin{array}{r}--Z-Y \\
E E F\end{array}$ & $\begin{array}{r}10 / 10 / 87 \\
18.36\end{array}$ \\
\hline $\begin{array}{l}V E=- \\
V E=\end{array}$ & $\begin{array}{r}3 . \equiv 22 z \\
3.2287\end{array}$ & $\begin{array}{r}: 5.941 E \\
0.28: 2\end{array}$ & $\begin{array}{r}--\bar{c} 22=\varepsilon \\
2.2227\end{array}$ & $\begin{array}{r}154.20 z: \\
0.0248\end{array}$ & 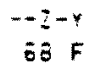 & $\begin{array}{r}10 .: 8 / \varepsilon^{2} \\
18: \geq 9\end{array}$ \\
\hline$\left\{\begin{array}{l}\because E: 3 \\
\because: \div=\end{array}\right.$ & z. 2225 & $\begin{array}{c}32.5555 \\
\text { 2.20 }\end{array}$ & $\begin{array}{r}-E: .=2: 3 \\
\text { a. } 2027\end{array}$ & $\begin{array}{r}11.4=2 \\
0.0014\end{array}$ & $\begin{array}{r}--z-y \\
3 E=\end{array}$ & $\begin{array}{r}10: 12 / 0^{-} \\
: 9: 2:\end{array}$ \\
\hline $\begin{array}{l}n:= \\
\therefore:=\end{array}$ & $\begin{array}{r}-Z 52=4 \\
2.2204\end{array}$ & 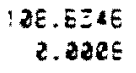 & $\begin{array}{r}\text { A2. } 2 E E 7 \\
\text { 2. } 22 R E\end{array}$ & 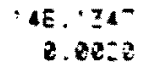 & $\begin{array}{l}--z-y \\
50=\end{array}$ & $\begin{array}{r}10,10: 8^{-} \\
: 5: 4^{-}\end{array}$ \\
\hline 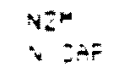 & $\begin{array}{r}3 E .-2= \\
\text { 2. } 2223\end{array}$ & $\begin{array}{r}05.1576 \\
3.2828\end{array}$ & $\begin{array}{r}-20=5 \\
2.2004\end{array}$ & $\begin{array}{r}58.285 z \\
\text { a.zoez }\end{array}$ & $\begin{array}{r}--i-z \\
-8 B=\end{array}$ & $\begin{array}{r}10,10 / 87 \\
9 \\
9=0\end{array}$ \\
\hline $\begin{array}{l}N 4= \\
B:=E\end{array}$ & $\begin{array}{r}-25.522 ! \\
3.2824\end{array}$ & $\begin{array}{r}08.2754 \\
2.2025\end{array}$ & $\begin{array}{r}-e .573 \\
\partial .2004\end{array}$ & $\begin{array}{r}77.64 ! 9 \\
0.82=9\end{array}$ & $\begin{array}{l}--i-\psi \\
56=\end{array}$ & $\begin{array}{r}10 / 18 / 87 \\
19: 5 \equiv\end{array}$ \\
\hline $\begin{array}{l}v+z \\
i:=z=\end{array}$ & $\begin{array}{r}-3.535 \\
8.2004\end{array}$ & $\begin{array}{r}104.2233 \\
3.32: 0\end{array}$ & $\begin{array}{r}4.5111 \\
0.8005\end{array}$ & $\begin{array}{r}42.85-3 \\
0.8024\end{array}$ & $\begin{array}{l}-z-\gamma \\
-z 8 F\end{array}$ & $\begin{array}{r}101: 8: 87 \\
: 5: 57\end{array}$ \\
\hline $\begin{array}{l}N-E=\bar{F} \\
P i=A=\end{array}$ & $\begin{array}{r}-1 \equiv .5945 \\
7.2204\end{array}$ & $\begin{array}{r}83.7134 \\
0.0210\end{array}$ & $\begin{array}{r}95.5484 \\
0.2811\end{array}$ & $\begin{array}{r}110.18: 1 \\
0.0223\end{array}$ & $\begin{array}{l}--i-\gamma \\
-8 B F\end{array}$ & $\begin{array}{r}: 18 / 87 \\
28: 16\end{array}$ \\
\hline $\begin{array}{l}N E \equiv A \\
N C F E\end{array}$ & $\begin{array}{r}14.2119 \\
2.2204\end{array}$ & $\begin{array}{r}93.7 \times 77 \\
8.081 !\end{array}$ & $\begin{array}{r}55.2277 \\
0.2812\end{array}$ & $\begin{array}{r}97.9252 \\
0.22 E\end{array}$ & $\begin{array}{r}--z-\gamma \\
68 F\end{array}$ & $\begin{array}{r}: 018 / 87 \\
28: 18\end{array}$ \\
\hline $\begin{array}{l}N 1 O \\
N \text { SAP }\end{array}$ & $\begin{array}{l}\text { 2. } 7810 \\
\text { e. } 2024\end{array}$ & $\begin{array}{r}.00 .2020 \\
0.2028\end{array}$ & $\begin{array}{r}92.2530 \\
\text { c.2010 }\end{array}$ & $\begin{array}{r}47.25 \equiv 59 \\
0.88=8\end{array}$ & $\begin{array}{r}-2-y \\
6 E F\end{array}$ & $\begin{array}{r}181: 0 / 87 \\
28: 21\end{array}$ \\
\hline $\begin{array}{l}N 20 \\
N=A P\end{array}$ & $\begin{array}{r}32.45 \equiv 3 \\
2.0007\end{array}$ & $\begin{array}{r}95.3141 \\
0.0811\end{array}$ & $\begin{array}{r}-3.5706 \\
0.0005\end{array}$ & $\begin{array}{r}129.7876 \\
2.2249\end{array}$ & $\begin{array}{r}--Z-Y \\
58 F\end{array}$ & $\begin{array}{r}10: 10 / 87 \\
20: 26\end{array}$ \\
\hline $\begin{array}{l}N Z M \\
N=A F\end{array}$ & $\begin{array}{r}-2.7494 \\
2.0004\end{array}$ & $\begin{array}{r}108.1925 \\
8.2028\end{array}$ & $\begin{array}{r}-48.5692 \\
8.2023\end{array}$ & $\begin{array}{r}98.8579 \\
\text { e.2815 }\end{array}$ & $\begin{array}{r}--Z+Y \\
68 F\end{array}$ & $\begin{array}{r}10 / 10 / 87 \\
20: 29\end{array}$ \\
\hline
\end{tabular}




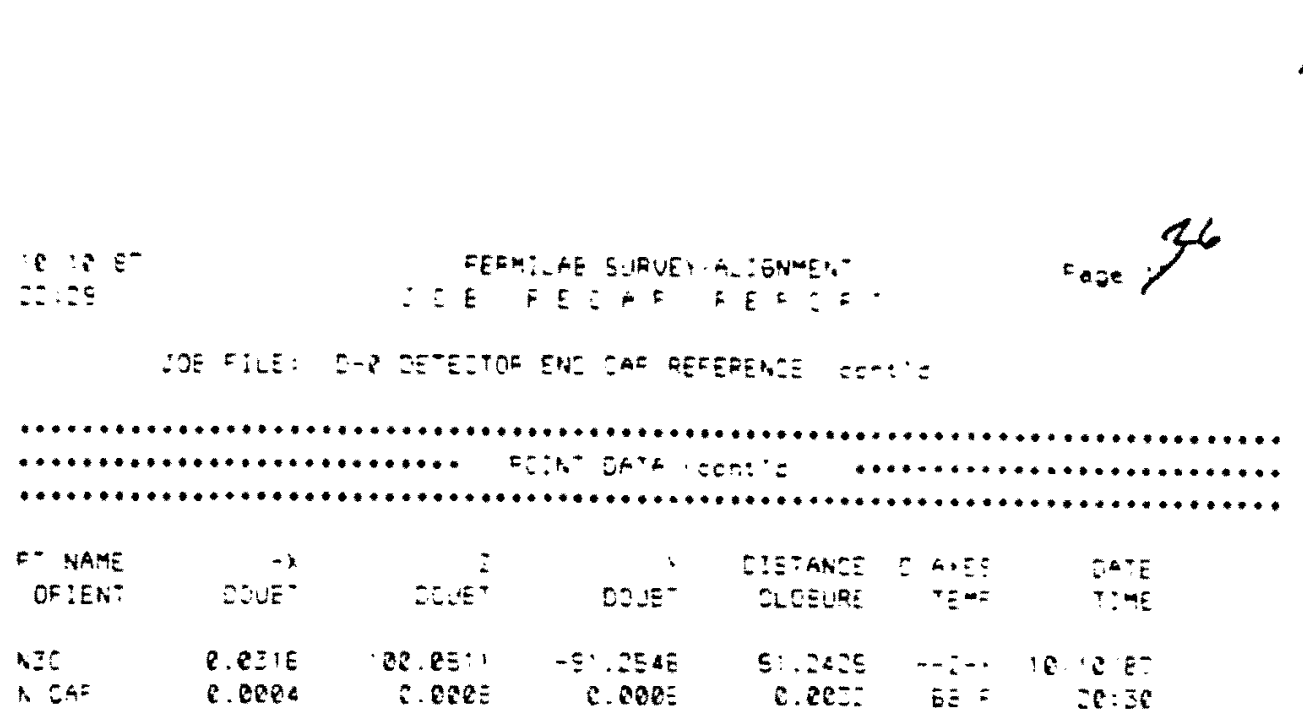

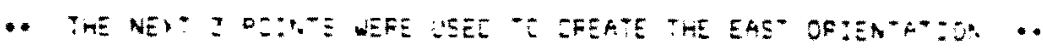

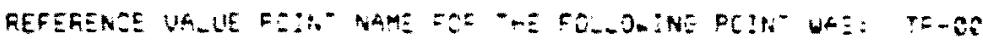

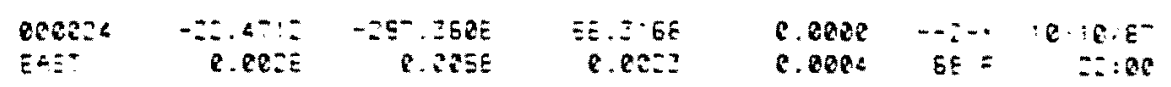

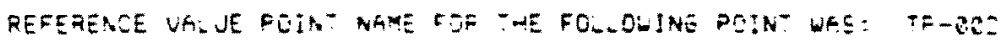

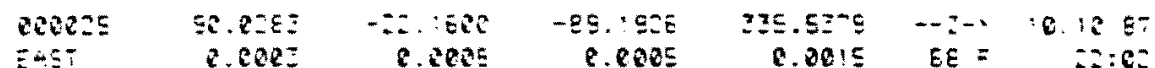

REFERENCE UALUE EEIST NAME FOF THE FOLSOLNE FOINT WAS: TF-OOU

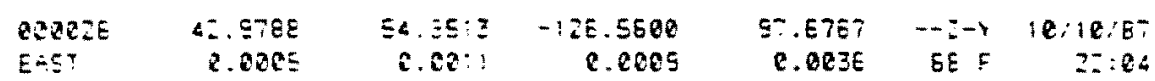

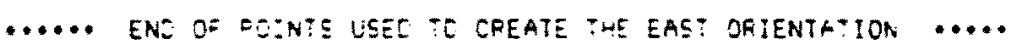

\begin{tabular}{|c|c|c|c|c|c|c|}
\hline $\begin{array}{l}\text { SEE: } \\
\text { SES" }\end{array}$ & $\begin{array}{r}-5.5 Z Z 5 \\
\text { C.DDeE }\end{array}$ & $\begin{array}{r}22.60= \\
\text { C. } 002=\end{array}$ & $\begin{array}{r}-6.555: \\
\text { c.0025 }\end{array}$ & $\begin{array}{r}E \equiv . E^{-3 E} \\
\text { R. } 2 e^{\prime}=\end{array}$ & $\begin{array}{c}-\overline{-Z} \\
-\overline{B E}=\end{array}$ & ie \\
\hline $\begin{array}{l}\text { NEETET } \\
\text { EAST }\end{array}$ & $\begin{array}{r}-5.713 \Xi \\
\text { 2.082 }\end{array}$ & $\begin{array}{r}-E .5 e: 2 \\
\text { C.02or }\end{array}$ & $\begin{array}{r}- \text { E: } \\
\text { e.cers }\end{array}$ & $\begin{array}{r}5 E . Z E E: \\
\text { e.ce: }\end{array}$ & $\begin{array}{r}-\because \overline{-} \\
E \bar{E}=\end{array}$ & ; \\
\hline
\end{tabular}

CHEQK EAST SIOE TCL BALLS FRO DIFF OPIENTAT:ON

\begin{tabular}{|c|c|c|c|c|c|c|}
\hline $\begin{array}{l}\text { EALNE } \\
\text { EAE" }\end{array}$ & $\begin{array}{r}62.7595 \\
\text { 2.cees }\end{array}$ & $\begin{array}{r}7 \equiv .5: 75 \\
\text { e. } 0008\end{array}$ & $\begin{array}{r}-75.0002 \\
2.0005\end{array}$ & $\begin{array}{l}4.285 \\
\text { e.ce: }\end{array}$ & $E \equiv$ & $\begin{array}{r}18 \cdot 10: \varepsilon \\
\quad 20\end{array}$ \\
\hline $\begin{array}{l}\text { EAL:SE } \\
\text { EAS: }\end{array}$ & $\begin{array}{r}5 e .594 \mathrm{~g} \\
e .000 \mathrm{E}\end{array}$ & $\begin{array}{r}28.270 ! \\
6.0002\end{array}$ & $\begin{array}{r}-7.9155 \\
6.0004\end{array}$ & $\begin{array}{r}55 . E \equiv z: \\
\text { C.0e0s }\end{array}$ & $\begin{array}{r}-\div-: \\
6 E:=\end{array}$ & $\begin{array}{r}10: 18: \varepsilon^{-} \\
2: 25\end{array}$ \\
\hline
\end{tabular}

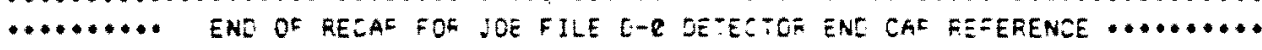

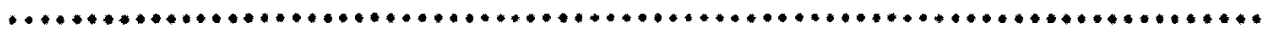




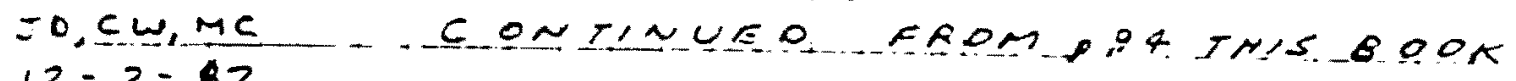
$12-2-8 ?$

BK D. $0 ., 25-1$.

WEST SIDE

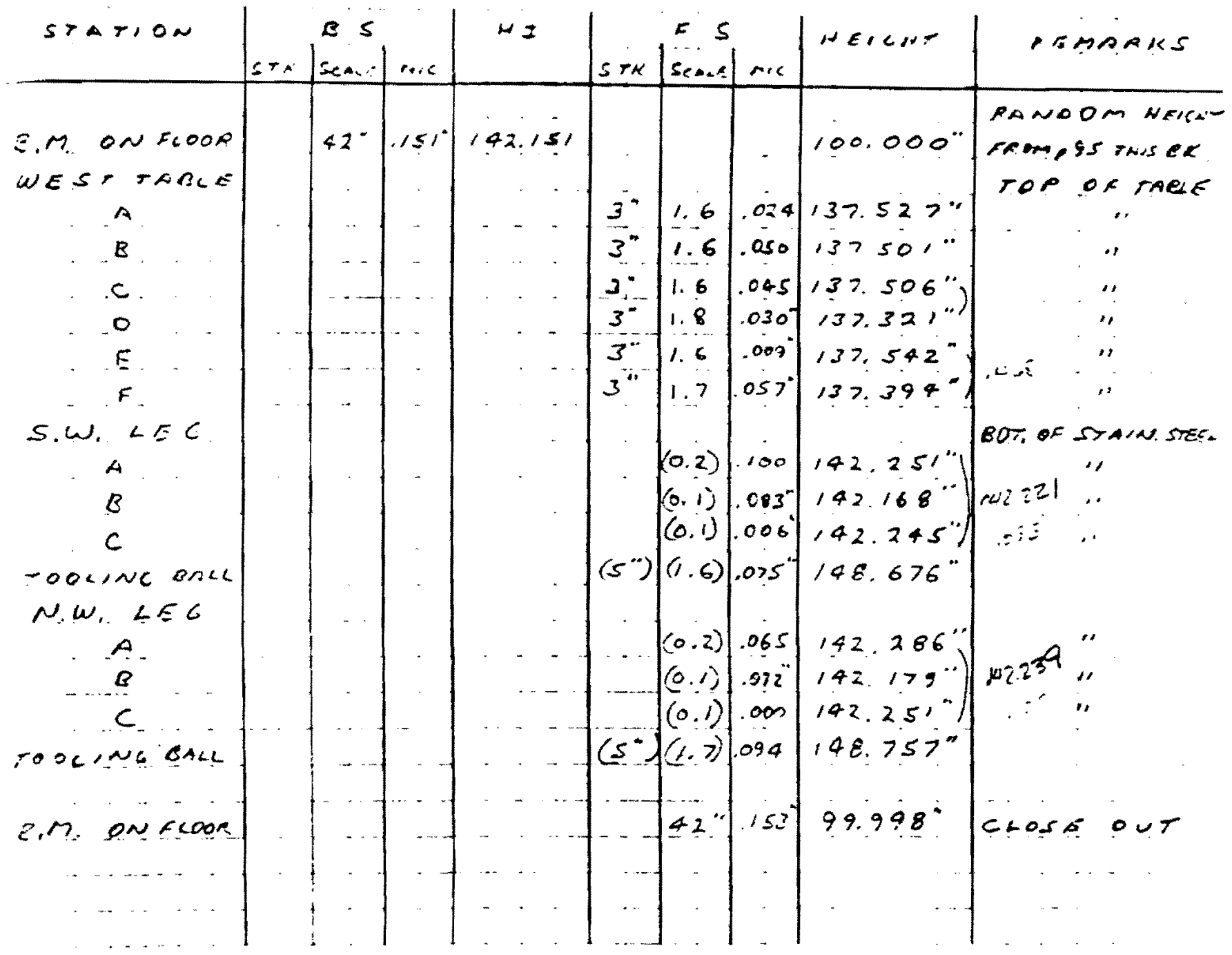
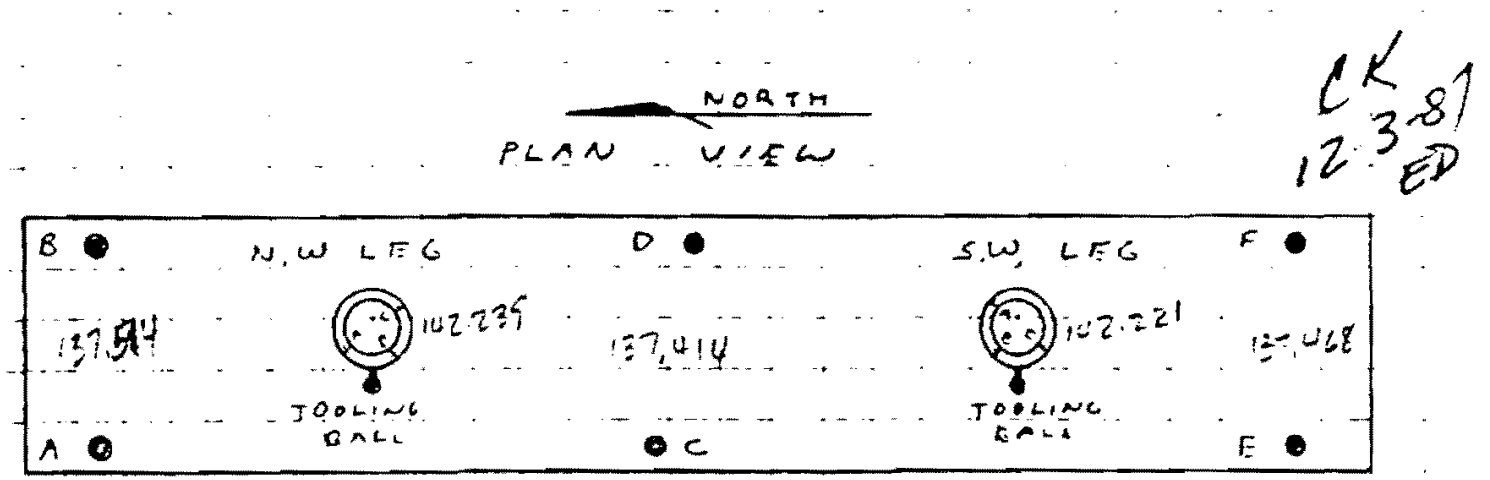
96

TD, Cw, MC

CONTINUEO FAOM. P. 95 THIS B.K.

$12-2 \cdot 87$

$8 K \quad 0.9,75-1$

HEICNT OR BOSSES

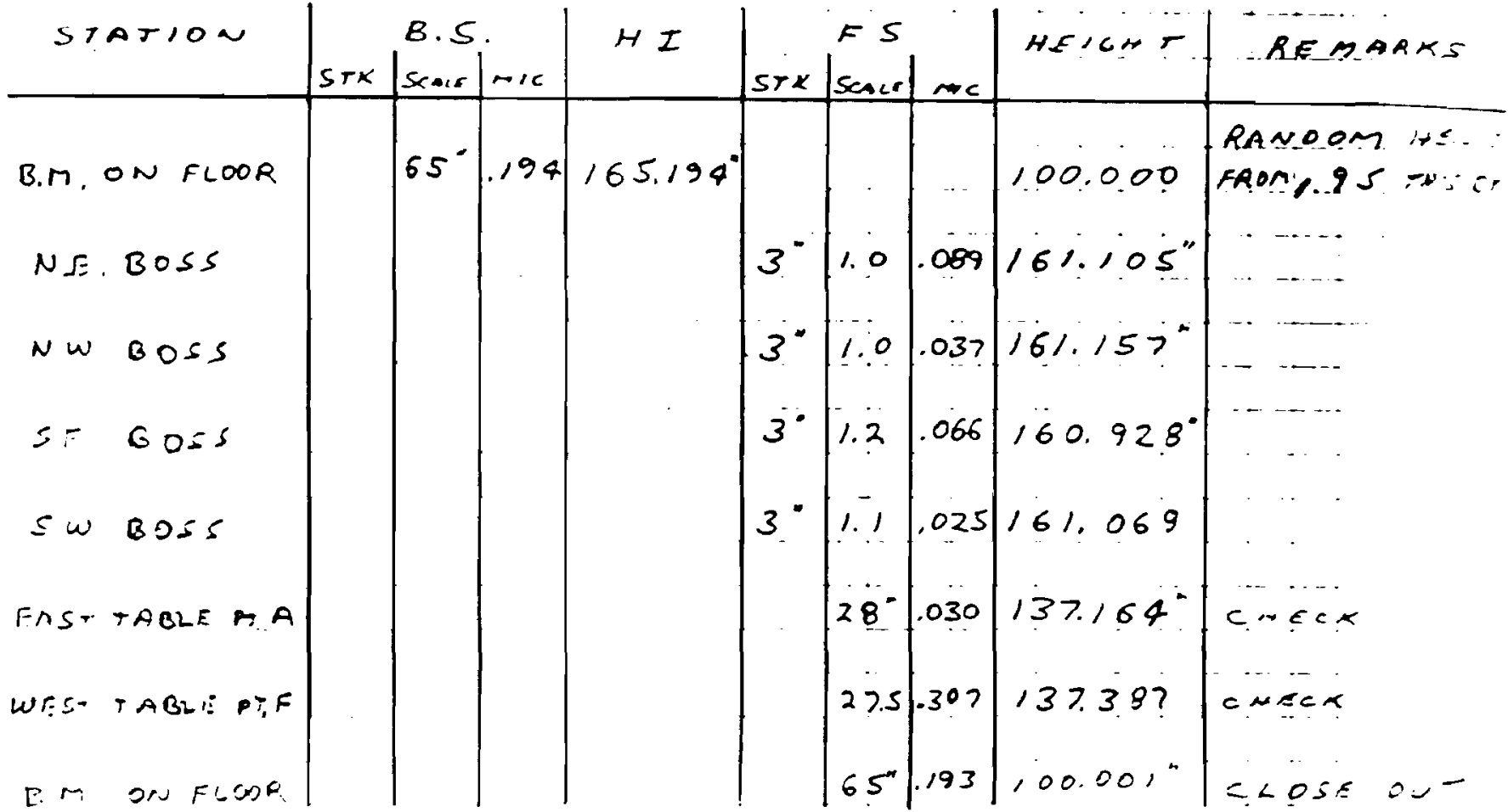

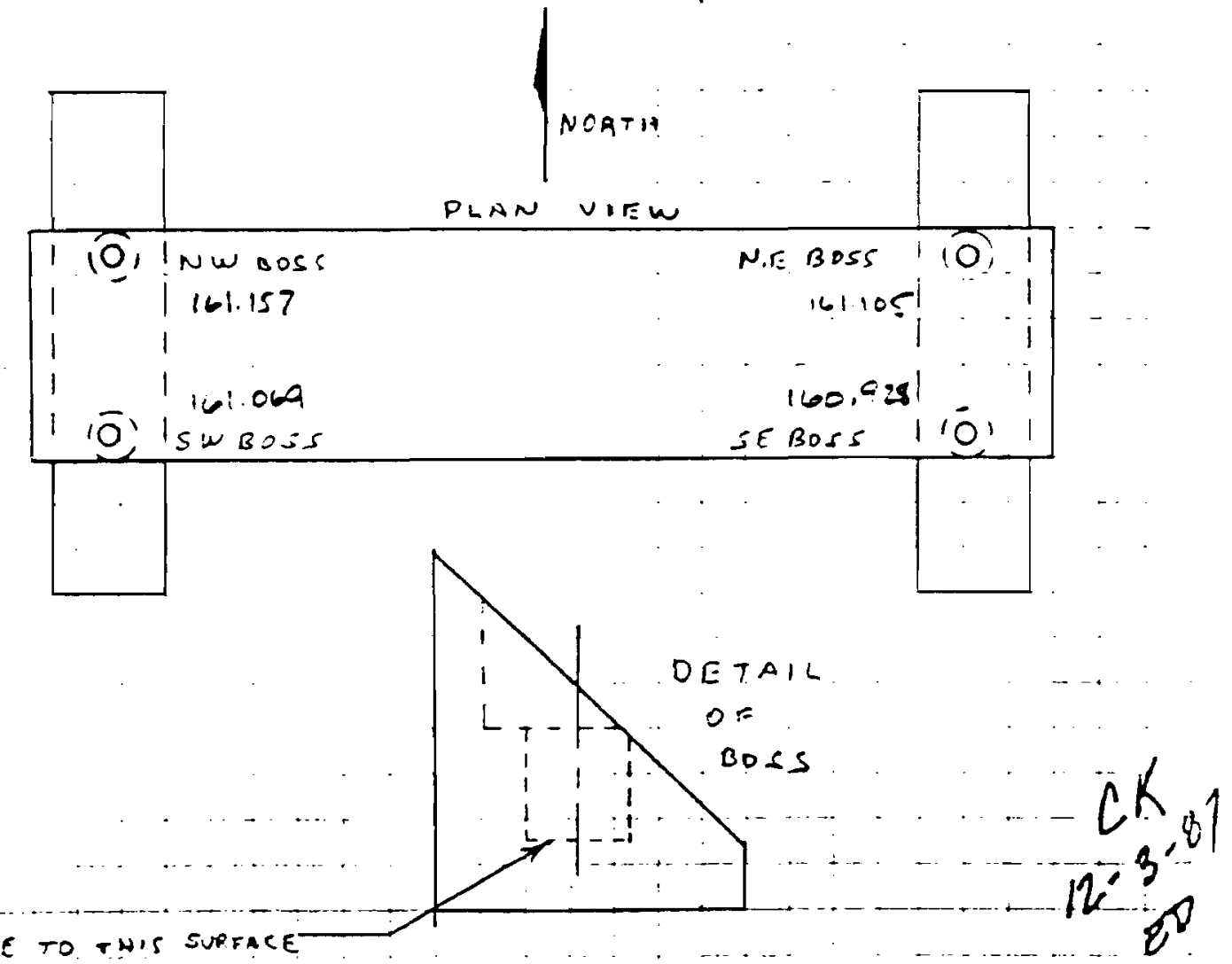

MEASURE 


$$
4-35-97
$$

IDCWIMC SUMMART. OF BOSS TO LEG DLECERANCES $12=2-87$ FROM P. 94,95, 96 TN/S Book

OK $0,0.75-1$.

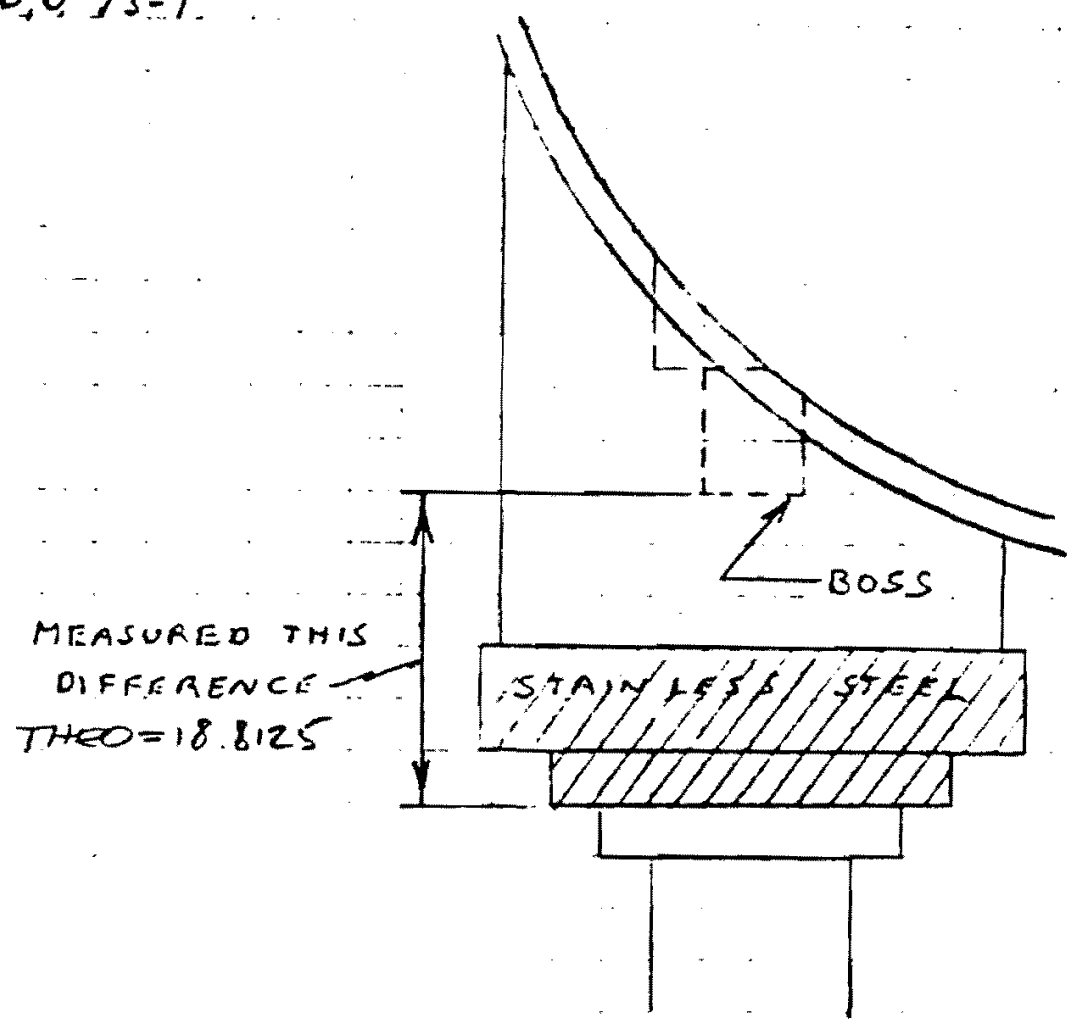

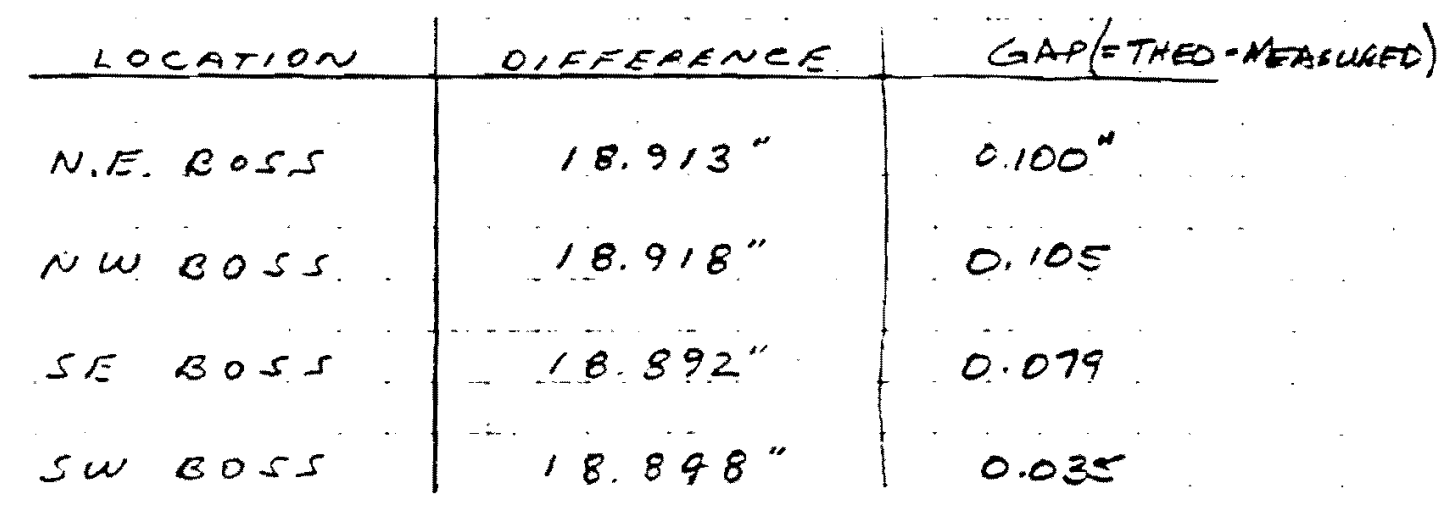

$$
\frac{C^{k}, 81}{1^{2}, 8}
$$


7.8

$A-36$

D, $\subset \omega, M=$

i2.3.87

BK D.0., $75-1$
MEASUPE TME NOZZLEE ON TOP DR THE.CFNTEF VESEEL RELATIUE TO A CONMON HOPZ RLANE SURUFN OONE IN LAEA.

SEE P.99 YWIS 82 FOL LOCATION ON PONSS. SURUFYC

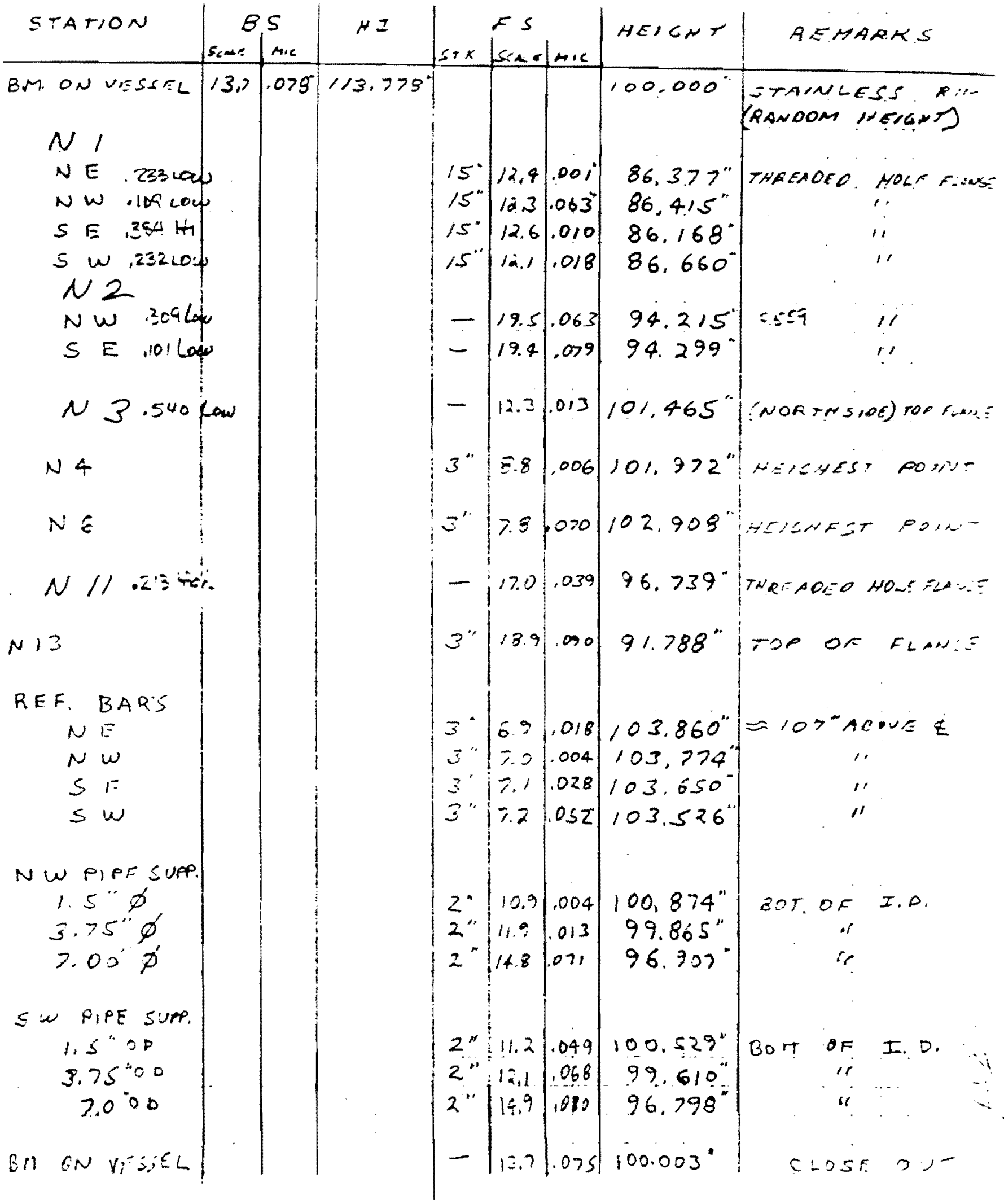


JD LOCAMION OF NOZZLES SURURTEO ON $12 \cdot 9-87 \quad$ C.9. Y4.S B.0.K

$B K 0.0,75-1$

NORTIS

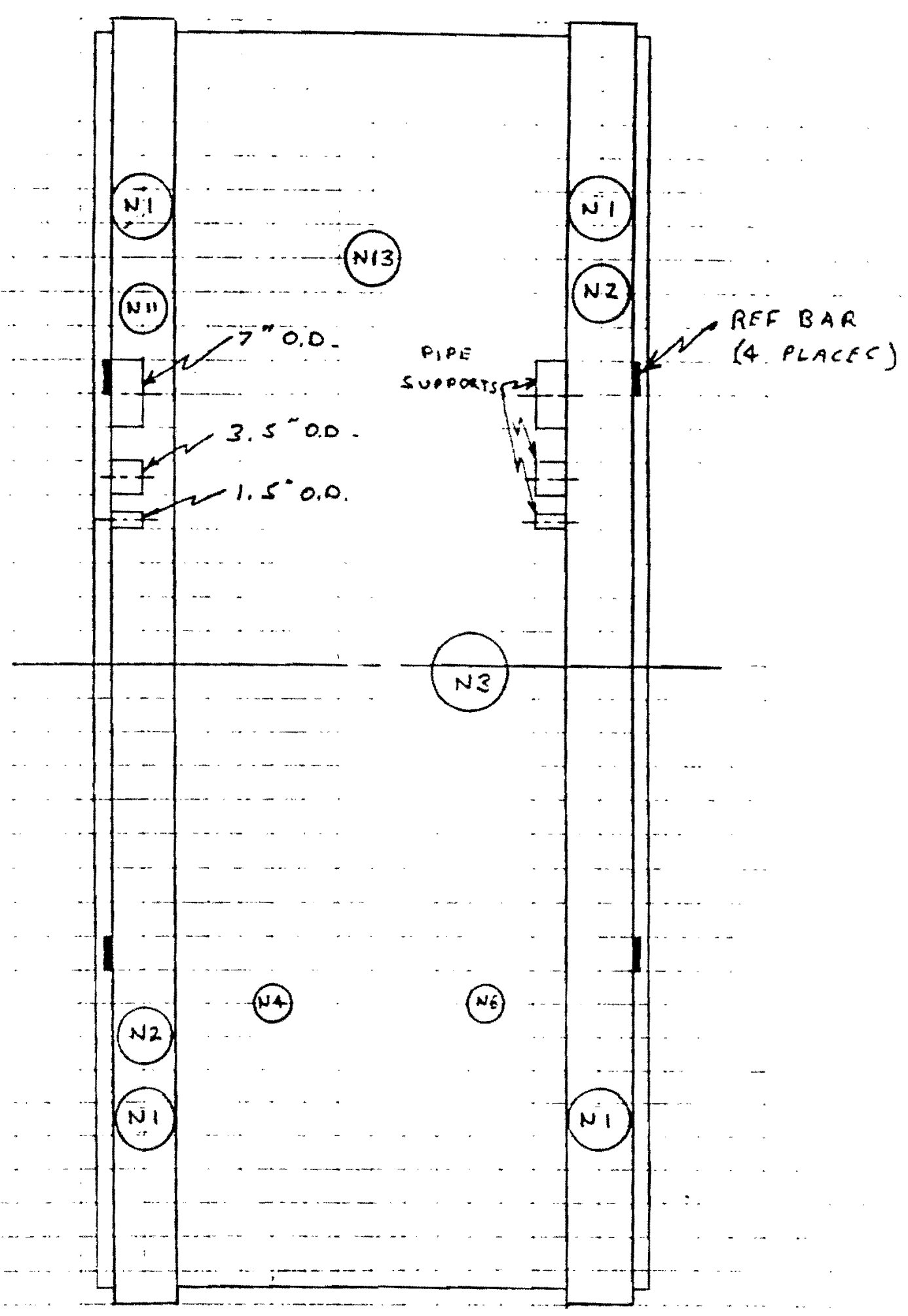


200

U.CW,MC HORZ, OISTANCES TO NOZZLES PIRE SUPPOKTS

12.5-87 ANO REF BARS LOCATEO ON TOP OT VESSEL BK $0.0,75-1$ ACCURACT IS $工$. 100".

NOTE: ALL MEASUREMENTS ART FROM

A LINE OEFINEO BI EXISTINC

PUNCN MAAKS ON TOP OF VESSEL.

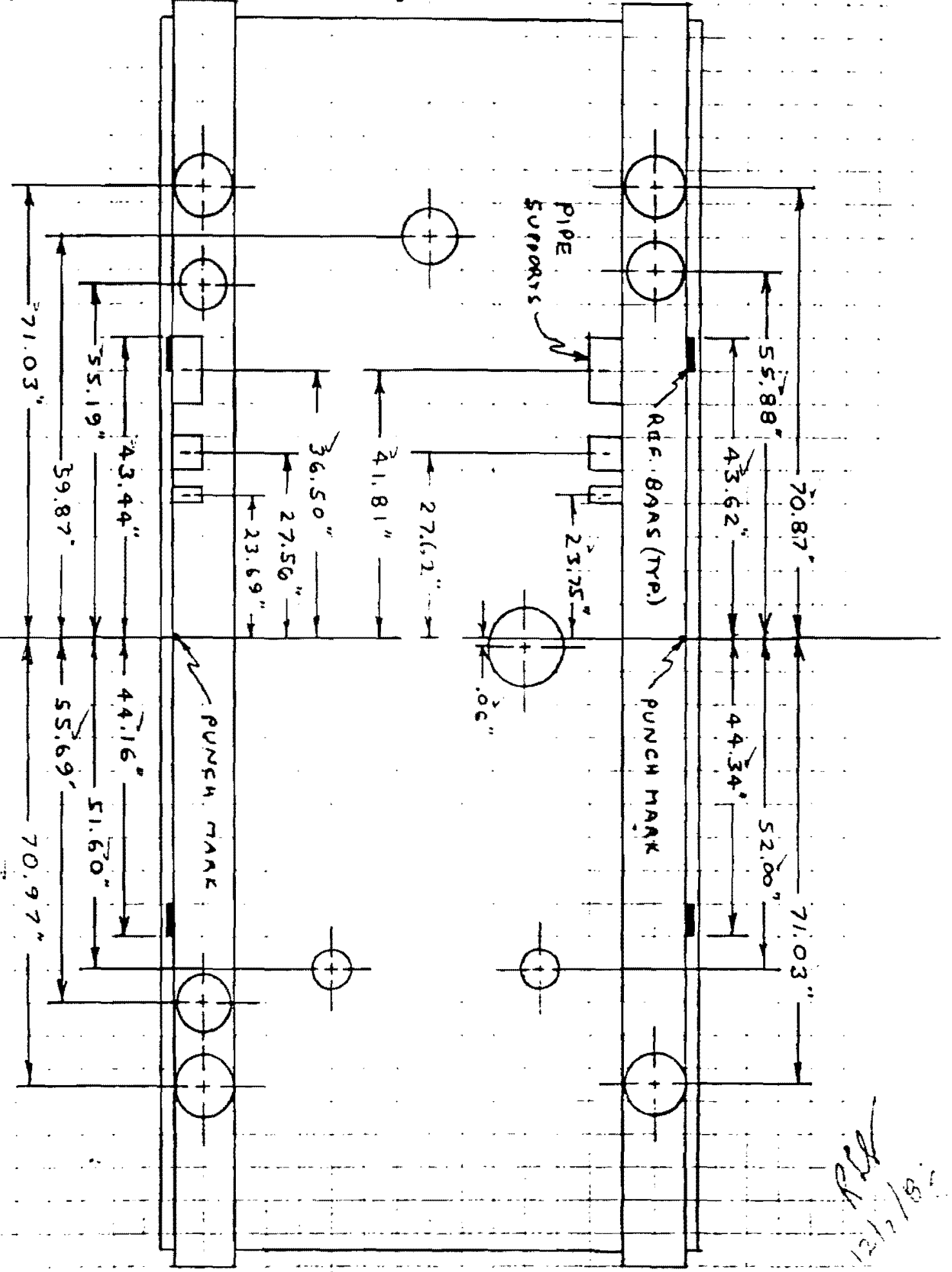


KSA L JOT

50. SCAATCH NOTSS POR P IOP THIS BK

$12-5=87$
$8 K$ DO $75-1$

RDC 11/2ays?

BARS ARE $1.5 \%$

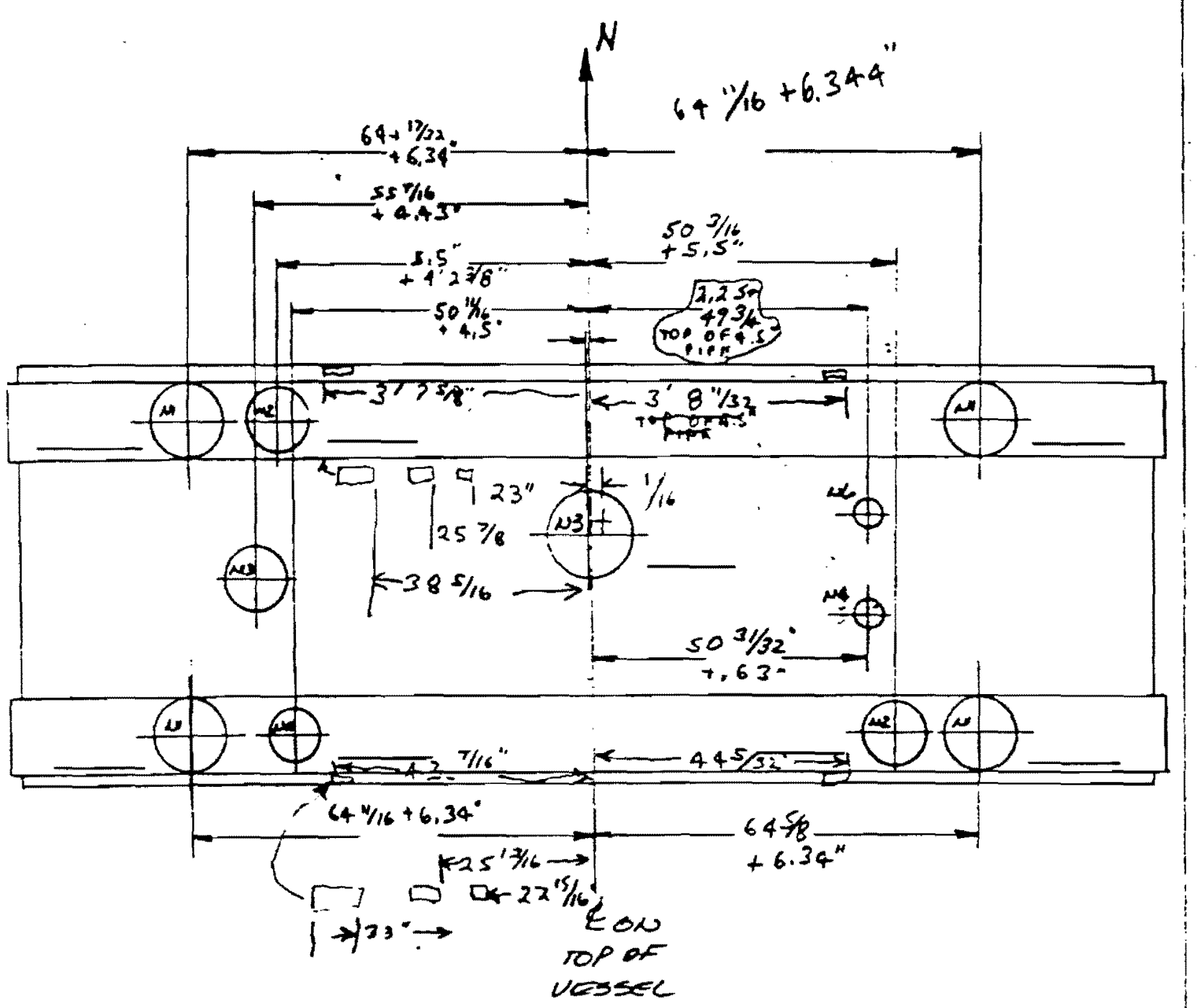

ASBUILT NOZZLE

locations

mexsues of: 